\title{
Vicarious Nucleophilic Chloromethylation of Nitroaromatics
}

Viktor V. Khutorianskyi, Blanka Klepetářová, Petr Beier*

Institute of Organic Chemistry and Biochemistry, Academy of Sciences of the Czech Republic, Flemingovo nám. 2, 16610 Prague, Czech Republic

E-mail:beier@uochb.cas.cz

\section{Supplementary information}

\section{Contents}

General information

Synthesis of compounds 2; General procedure A

Synthesis of compounds 2; General procedure B

$\mathrm{S}_{\mathrm{N}} \mathrm{Ar}$ reactions with substituted nitrobenzenes

SI8

Synthesis of compounds 4 by a one-pot chloromethylation/alkylation sequence; General procedure $\mathrm{C}$

Synthesis of compounds 5 by a one-pot chloromethylation/oxidation sequence; General procedure $\mathrm{D}$ SI9

Crystallographic data SI11

References SI12

Copies of NMR spectra SI13

\section{General information}

NMR chemical shifts $(\delta)$ are reported in ppm and coupling constants $(J)$ are given in Hertz and referenced to residual signals of solvents or internal standards: $\mathrm{CDCl}_{3} \delta_{\mathrm{H}}=7.26, \delta_{\mathrm{C}}=$ 77.16; $\mathrm{Me}_{4} \mathrm{Si} \delta_{\mathrm{H}}=0.00 ; \mathrm{CFCl}_{3} \delta_{\mathrm{F}}=0.00 .{ }^{13} \mathrm{C}$ and ${ }^{19} \mathrm{~F}$ NMR spectra were ${ }^{1} \mathrm{H}$ decoupled. GCMS spectra were recorded on a gas chromatograph coupled with a quadrupole massselective electron impact (EI) detector $(70 \mathrm{eV})$. High-resolution mass spectra (HRMS) were recorded on a gas chromatograph coupled with an orthogonal acceleration time-of-flight detector using EI ionization or an FT mass spectrometer using electrospray (ESI) ionization. Infrared spectra were measured on a FT-IR instrument. Purification of the products was 
performed by flash chromatography using silica gel 60. Dry solvents if used were obtained the following way: $\mathrm{Et}_{2} \mathrm{O}$ and THF were distilled over $\mathrm{Na} /$ benzophenone and kept over activated $3 \AA$ molecular sieves, hexane and DCM were dried using activated $3 \AA$ molecular sieves, TMEDA was dried and kept over $\mathrm{KOH}$. PE refers to petroleum ether of boiling point range $40-60{ }^{\circ} \mathrm{C}$.

Synthesis of compounds 2; General procedure A. A solution of $n$-BuLi (2.5 M, $1.6 \mathrm{~mL}, 4$ mmol, 4 equiv.) in hexanes was added over 5 min to a solution of $\mathrm{CH}_{2} \mathrm{Cl}_{2}$ (4 mmol, 4 equiv.) in dry THF $(8 \mathrm{~mL})$ cooled to $-100{ }^{\circ} \mathrm{C}$ under argon. After stirring the mixture for $15 \mathrm{~min}$, the resulting solution was added via syringe to a solution of nitroaromatic $\mathbf{1}$ ( $1 \mathrm{mmol}, 1$ equiv.) in dry THF $\left(8 \mathrm{~mL}\right.$ ) cooled to $-100{ }^{\circ} \mathrm{C}$ (liquid $\left.\mathrm{N}_{2} / \mathrm{EtOH}\right)$ under argon. The resulting mixture was stirred at this temperature for $30 \mathrm{~s}$, followed by the addition of cold $\left(0{ }^{\circ} \mathrm{C}\right)$ solution of $\mathrm{CF}_{3} \mathrm{SO}_{3} \mathrm{H}(0.45 \mathrm{ml}, 5 \mathrm{mmol}, 5$ equiv. $)$ in $\mathrm{H}_{2} \mathrm{O}(3-5 \mathrm{~mL})$. The cooling bath was removed and the mixture was slowly warmed to rt. The reaction mixture was extracted with $\mathrm{Et}_{2} \mathrm{O}$, the combined organic phase was washed with water, brine, dried $\left(\mathrm{MgSO}_{4}\right)$ and solvent was removed under reduced pressure. Purification using flash chromatography (silica gel, hexane $/ \mathrm{CH}_{2} \mathrm{Cl}_{2}$ ) provided pure product 2 .

3-(Chloromethyl)-4-nitrophenyl)pentafluoro- $\lambda^{6}$-sulfane (2a). Prepared from 4-nitro-1-<smiles>O=[N+]([O-])c1ccc([18F])cc1CCl</smiles(pentafluorosulfanyl)benzene following the General procedure A. Yellow oil (182 $\mathrm{mg}, 61 \%$ yield); $R_{f} 0.46$ (cyclohexane/Et $\left.2 \mathrm{O}, 95: 5\right) ;{ }^{1} \mathrm{H} \mathrm{NMR}\left(\mathrm{CDCl}_{3}, 400 \mathrm{MHz}\right) \delta$ 8.17-8.11 (2H, m), $7.91(1 \mathrm{H}, \mathrm{dd}, J=8.9,2.4 \mathrm{~Hz}), 4.99(1 \mathrm{H}, \mathrm{s}) ;{ }^{13} \mathrm{C} \mathrm{NMR}\left(\mathrm{CDCl}_{3}\right.$, $100 \mathrm{MHz}) \delta 156.71(\mathrm{t}, J=19.2 \mathrm{~Hz}$ ), 149.26, 133.79, 129.58 (quint, $J=4.6 \mathrm{~Hz}$ ), 127.49 (quint, $J=4.8 \mathrm{~Hz}), 125.89,42.07 ;{ }^{19} \mathrm{~F} \mathrm{NMR}\left(\mathrm{CDCl}_{3}, 376 \mathrm{MHz}\right) \delta 80.40(1 \mathrm{~F}, \mathrm{~m}), 62.05(4 \mathrm{~F}, \mathrm{~d}, J=$ $150.9 \mathrm{~Hz}$ ); GCMS (EI) m/z 278 (9\%), 262 (24) [M-Cl] , 246 (6), 231 (21), 218 (47), 123 (33), 110 (48), 108 (47), 107 (70), 89 (100), 83 (53), 75 (37), 63 (75), 62 (37); HRMS (CI+) m/z Calcd for $\mathrm{C}_{7} \mathrm{H}_{5} \mathrm{ClF}_{5} \mathrm{NO}_{2} \mathrm{~S}[\mathrm{M}]^{+}:$296.9650; Found: 296.9656.

4-(Chloromethyl)-3-nitrophenyl)pentafluoro- $\lambda^{6}$-sulfane (2b). Prepared from 3-nitro-1$\mathrm{NO}_{2} \quad$ (pentafluorosulfanyl)benzene following the General procedure A. Yellow oil (188 mg, 63\% yield); $R_{f} 0.4$ (cyclohexane/Et $\left.2 \mathrm{O}, 95: 5\right) ;{ }^{1} \mathrm{H} \mathrm{NMR}\left(\mathrm{CDCl}_{3}, 400\right.$ MHz) $\delta 8.70(1 \mathrm{H}, \mathrm{d}, J=2.3 \mathrm{~Hz}), 8.40(1 \mathrm{H}, \mathrm{dd}, J=8.6,2.2 \mathrm{~Hz}), 7.96(1 \mathrm{H}, \mathrm{d}, J$ $=8.6 \mathrm{~Hz}), 4.95(2 \mathrm{H}, \mathrm{s}) ;{ }^{13} \mathrm{C} \mathrm{NMR}\left(\mathrm{CDCl}_{3}, 100 \mathrm{MHz}\right) \delta 153.83-153.16(\mathrm{~m}), 147.56,136.25$, 132.25, 130.94 (quint, $J=4.5 \mathrm{~Hz}$ ), 123.53 (quint, $J=4.9 \mathrm{~Hz}$ ), 42.02; ${ }^{19} \mathrm{~F} \mathrm{NMR}\left(\mathrm{CDCl}_{3}, 376\right.$ MHz) $\delta 81.75(1 \mathrm{~F}, \mathrm{~m}), 67.11$ (4F, d, $J=150.0 \mathrm{~Hz}$ ); GCMS (EI) $m / z, 299$ (20\%) [M] ${ }^{+}, 297$ (53) $[\mathrm{M}]^{+}, 262$ (73), 171 (20), 169 (58), 168 (31), 143 (28), 123 (27), 107 (67), 96 (27), 89 (100), 
63 (43), 62 (23); HRMS (EI $\left.{ }^{+}\right) \mathrm{m} / z$ Calcd for $\mathrm{C}_{7} \mathrm{H}_{5} \mathrm{ClF}_{5} \mathrm{NO}_{2} \mathrm{~S}[\mathrm{M}]^{+}$: 296.9650; Found: 296.9652 .

(4-(Chloromethyl)-3-fluoro-5-nitrophenyl)pentafluoro- $\lambda^{6}$-sulfane (2c). Prepared from 3-nitro$\mathrm{NO}_{2}$ 5-fluoro-1-(pentafluorosulfanyl)benzene following the General procedure A. Cl Yellow amorphous solid $\left(192 \mathrm{mg}, 61 \%\right.$ yield); $R_{f} 0.47$ (cyclohexane/Et $2 \mathrm{O}$, 95:5); ${ }^{1} \mathrm{H} \mathrm{NMR}\left(\mathrm{CDCl}_{3}, 400 \mathrm{MHz}\right) \delta 8.27(1 \mathrm{H}, \mathrm{t}, J=2.0 \mathrm{~Hz}), 7.83(1 \mathrm{H}, \mathrm{dd}, J$ $=8.9,2.2 \mathrm{~Hz}), 4.95(2 \mathrm{H}, \mathrm{d}, J=1.7 \mathrm{~Hz}) ;{ }^{13} \mathrm{C} \mathrm{NMR}\left(\mathrm{CDCl}_{3}, 100 \mathrm{MHz}\right) \delta 160.20(\mathrm{~d}, J=258.4$ $\mathrm{Hz}), 148.71,125.14$ (d, $J=18.0 \mathrm{~Hz}), 119.57-119.23$ (2C, m), 119.23-118.94 (m), 32.95 (d, $J$ $=6.1 \mathrm{~Hz}) ;{ }^{19} \mathrm{~F} \mathrm{NMR}\left(\mathrm{CDCl}_{3}, 376 \mathrm{MHz}\right) \delta 79.49-77.87(1 \mathrm{~F}, \mathrm{~m}), 62.25(4 \mathrm{~F}, \mathrm{~d}, J=151.6 \mathrm{~Hz}),-$ $108.20\left(3 \mathrm{~F}, \mathrm{~d}, J=8.8 \mathrm{~Hz}\right.$ ); GCMS (EI) $m / z 317(6 \%)[\mathrm{M}]^{+}, 315(16 \%)[\mathrm{M}]^{+}, 280(50), 278$ (100), 249 (6), 248 (23), 143 (15), 141 (48), 126 (24), 125 (36), 107 (37) 89 (62) 81 (28); HRMS $\left(\mathrm{EI}^{+}\right) \mathrm{m} / z$ Calcd for $\mathrm{C}_{7} \mathrm{H}_{4} \mathrm{~F}_{6} \mathrm{NO}_{2} \mathrm{SCl}[\mathrm{M}]^{+}: 314.9555$; Found: 314.9553.

2-(Chloromethyl)-1-nitro-4-(trifluoromethyl)benzene (2c). Prepared from 1-nitro-4(trifluoromethyl)benzene following the General procedure A. Yellow oil $(172 \mathrm{mg}$,
$72 \%$ yield); $R_{f} 0.51$ (cyclohexane/Et $\left.2 \mathrm{O}, 95: 5\right) ;{ }^{1} \mathrm{H} \mathrm{NMR}\left(\mathrm{CDCl}_{3}, 400 \mathrm{MHz}\right) \delta 8.16$
$(1 \mathrm{H}, \mathrm{d}, J=8.5 \mathrm{~Hz}), 8.00(1 \mathrm{H}, \mathrm{s}), 7.79(1 \mathrm{H}, \mathrm{d}, J=8.5 \mathrm{~Hz}), 5.00(2 \mathrm{H}, \mathrm{s}) ;{ }^{13} \mathrm{C} \mathrm{NMR}$ $\left(\mathrm{CDCl}_{3}, 100 \mathrm{MHz}\right) \delta 150.09,135.43(\mathrm{q}, J=33.7 \mathrm{~Hz}), 133.64,128.88(\mathrm{q}, J=3.6 \mathrm{~Hz}), 126.68$ $(\mathrm{q}, J=3.6 \mathrm{~Hz}), 125.95,122.81(\mathrm{q}, J=273.3 \mathrm{~Hz}), 42.11 ;{ }^{19} \mathrm{~F} \mathrm{NMR}\left(\mathrm{CDCl}_{3}, 376 \mathrm{MHz}\right) \delta-$ 63.23 (3F, s); GCMS (EI) m/z 220 (10\%) [M-F] $]^{+}, 204$ (21) [M-Cl], 194 (5), 176 (22), 160 (100), 145 (31), 137 (14), 107 (14), 89 (12), 63 (21); HRMS (CI) m/z Calcd for $\mathrm{C}_{8} \mathrm{H}_{6} \mathrm{~F}_{3} \mathrm{NO}_{2} \mathrm{Cl}$ $[\mathrm{M}+\mathrm{H}]^{+}:$240.0039; Found: 240.0038 .

1-(Chloromethyl)-2-nitro-4-(trifluoromethyl)benzene (2e). ${ }^{1}$ Prepared from 1-nitro-3(trifluoromethyl)benzene following the General procedure A. Yellow $\mathrm{Cl}_{\mathrm{Cl}}$ amorphous solid (184 mg, 77\% yield); $R_{f} 0.49$ (cyclohexane/Et $\left.{ }_{2} \mathrm{O}, 95: 5\right) ;{ }^{1} \mathrm{H}$ NMR $\left(\mathrm{CDCl}_{3}, 400 \mathrm{MHz}\right) \delta 8.58(1 \mathrm{H}, \mathrm{s}), 8.47(1 \mathrm{H}, \mathrm{d}, J=8.6 \mathrm{~Hz}), 7.95(1 \mathrm{H}, \mathrm{d}$, $J=8.4 \mathrm{~Hz}), 4.84(2 \mathrm{H}, \mathrm{s}) ;{ }^{13} \mathrm{C} \mathrm{NMR}\left(\mathrm{CDCl}_{3}, 100 \mathrm{MHz}\right) \delta 142.61,133.14,127.19,124.24$, 121.96-121.75 (m), 121.51, 40.89 (q, $J=2.9 \mathrm{~Hz}) ;{ }^{19} \mathrm{~F}$ NMR $\left(\mathrm{CDCl}_{3}, 376 \mathrm{MHz}\right) \delta-58.88(3 \mathrm{~F}$, s); GCMS (EI) m/z 240 (4\%) [M] , 238 (13) [M] $]^{+}, 202$ (100), 191 (9), 179 (12), 172 (31), 157 (19), 75 (9); HRMS (CI) $m / z$ Calcd for $\mathrm{C}_{8} \mathrm{H}_{6} \mathrm{~F}_{3} \mathrm{NO}_{2} \mathrm{Cl}[\mathrm{M}+\mathrm{H}]^{+}:$240.0039; Found: 240.0038. 4-(Chloromethyl)-1-nitro-2-(trifluoromethyl)benzene (2f). Prepared from 1-nitro-2$\mathrm{NO}_{2} \quad$ (trifluoromethyl)benzene following the General procedure A. Yellow oil (113 mg, $\mathrm{CF}_{3} 47 \%$ yield); $R_{f} 0.17$ (cyclohexane/Et $\left.2 \mathrm{O}, 95: 5\right) ;{ }^{1} \mathrm{H} \mathrm{NMR}\left(\mathrm{CDCl}_{3}, 400 \mathrm{MHz}\right) \delta 7.90$ $C_{\mathrm{Cl}}(1 \mathrm{H}, \mathrm{d}, J=8.3 \mathrm{~Hz}), 7.85(1 \mathrm{H}, \mathrm{d}, J=1.8 \mathrm{~Hz}), 7.76(1 \mathrm{H}, \mathrm{dd}, J=8.3,1.9 \mathrm{~Hz}), 4.67$ 
$(2 \mathrm{H}, \mathrm{s}) ;{ }^{13} \mathrm{C} \mathrm{NMR}\left(\mathrm{CDCl}_{3}, 100 \mathrm{MHz}\right) \delta 152.91,142.82,132.92,128.10(\mathrm{q}, J=5.0 \mathrm{~Hz})$, 125.77, 120.47, 43.93; ${ }^{19} \mathrm{~F}$ NMR $\left(\mathrm{CDCl}_{3}, 376 \mathrm{MHz}\right) \delta-60.59$ (3F, s); GCMS (EI) $\mathrm{m} / z 239$ (34\%) [M] $]^{+}, 204$ (42), 181 (27), 158 (100), 145 (27), 138 (26), 63 (30); HRMS (CI) m/z Calcd for $\mathrm{C}_{8} \mathrm{H}_{5} \mathrm{ClF}_{3} \mathrm{NO}_{2}[\mathrm{M}+\mathrm{H}]^{+}: 240.0039$; Found: 240.0037 .

1-(Chloromethyl)-2,4-dinitrobenzene $(\mathbf{2 g})^{2}$. Prepared from 1,3-dinitrobenzene following the<smiles>O=[N+]([O-])c1ccc(CCl)c([N+](=O)[O-])c1</smiles>

General procedure A. Yellow amorphous solid (180 mg, 83\% yield); $R_{f} 0.14$

(cyclohexane/Et $\left.{ }_{2} \mathrm{O}, 95: 5\right) ;{ }^{1} \mathrm{H} \mathrm{NMR}\left(\mathrm{CDCl}_{3}, 400 \mathrm{MHz}\right) \delta 8.91(1 \mathrm{H}, \mathrm{d}, J=2.3$ $\mathrm{Hz}), 8.51(1 \mathrm{H}, \mathrm{dd}, J=8.5,2.3 \mathrm{~Hz}), 8.01(1 \mathrm{H}, \mathrm{d}, J=8.6 \mathrm{~Hz}), 5.06(2 \mathrm{H}, \mathrm{s}) ;{ }^{13} \mathrm{C}$ NMR $\left(\mathrm{CDCl}_{3}, 100 \mathrm{MHz}\right) \delta 148.12,147.85,138.92,132.99,127.91,120.81,42.04$; GCMS (EI) $m / z 181$ (38\%) [M-Cl], 153 (10), 150 (9), 137(24), 134 (35), 125 (20), 123 (41), 107 (67), 91 (84), 89 (100), 77 (54), 63 (99); HRMS (CI) m/z Calcd for $\mathrm{C}_{7} \mathrm{H}_{6} \mathrm{ClN}_{2} \mathrm{O}_{4}$ $[\mathrm{M}+\mathrm{H}]^{+}: 217.0016$; Found: 217.0014;

Synthesis of compounds 2; General procedure B. A solution of $n$-BuLi (2.5 M, $1.6 \mathrm{~mL}, 4$ mmol, 4 equiv.) in hexanes was added over 5 min to a solution of $\mathrm{CH}_{2} \mathrm{Cl}_{2}$ (4 mmol, 4 equiv.) in dry THF $(8 \mathrm{~mL})$ cooled to $-100{ }^{\circ} \mathrm{C}$ under argon. After stirring the mixture for $15 \mathrm{~min}$, the resulting solution was added via syringe to a solution of nitroaromatic 1 (153 mg, $1 \mathrm{mmol}, 1$ equiv.) and TMEDA ( $\sim 0.6 \mathrm{~mL}, \sim 4 \mathrm{mmol}, 4$ equiv.) in dry THF $(8 \mathrm{~mL})$ cooled to $-100{ }^{\circ} \mathrm{C}$ (liquid $\mathrm{N}_{2} / \mathrm{EtOH}$ ) under argon. The resulting mixture was stirred at this temperature for $6 \mathrm{~h}$, followed by the addition of saturated aqueous $\mathrm{NH}_{4} \mathrm{Cl}(3-5 \mathrm{~mL})$. The cooling bath was removed and the mixture was slowly warmed to $\mathrm{rt}$. The reaction mixture was extracted with $\mathrm{Et}_{2} \mathrm{O}$, the combined organic phase was washed with water, brine, dried $\left(\mathrm{MgSO}_{4}\right)$ and solvent was removed under reduced pressure. Purification using flash chromatography (silica gel, hexane $/ \mathrm{CH}_{2} \mathrm{Cl}_{2}$ ) provided pure product 2 .

3-(Chloromethyl)-4-nitrobenzonitrile $(\mathbf{2 h})$. Prepared from 4-nitrobenzonitrile following the<smiles>N#Cc1ccc([N+](=O)[O-])c(CCl)c1</smiles>

General procedure B. Yellow amorphous solid (171 mg, 87\% yield); $R_{f} 0.09$ (cyclohexane/Et $2 \mathrm{O}, 95: 5) ;{ }^{1} \mathrm{H} \mathrm{NMR}\left(\mathrm{CDCl}_{3}, 400 \mathrm{MHz}\right) \delta 8.15(1 \mathrm{H}, \mathrm{d}, J=8.4 \mathrm{~Hz})$, $8.07(1 \mathrm{H}, \mathrm{d}, J=1.8 \mathrm{~Hz}), 7.84(1 \mathrm{H}, \mathrm{dd}, J=8.4,1.8 \mathrm{~Hz}), 4.97(1 \mathrm{H}, \mathrm{s}) ;{ }^{13} \mathrm{C} \mathrm{NMR}$ $\left(\mathrm{CDCl}_{3}, 100 \mathrm{MHz}\right) \delta 135.29,134.02,133.10,126.03,117.76,116.49,41.76$; GCMS (EI) $\mathrm{m} / z$ $196(1 \%)[\mathrm{M}]^{+}, 161$ (39), 134 (13), 123 (33), 117 (100), 114 (86), 105 (31), 102 (46), 90 (91), 88 (10), 75 (29), 63 (48), 50 (24), 48 (8); HRMS (EI) m/z Calcd for $\mathrm{C}_{8} \mathrm{H}_{5} \mathrm{~N}_{2} \mathrm{O}_{2} \mathrm{Cl}[\mathrm{M}]^{+}$: 196.0040; Found: 196.0037. 
4-(Chloromethyl)-3-nitrobenzonitrile (2i). Prepared from 3-nitrobenzonitrile following the $\mathrm{NO}_{2} \quad$ General procedure B. Yellow solid (173 mg, 88\% yield); m.p. 84-85 ${ }^{\circ} \mathrm{C} ; R_{f}$ 0.24 (cyclohexane/Et $2 \mathrm{O}, 75: 25) ;{ }^{1} \mathrm{H} \mathrm{NMR}\left(\mathrm{CDCl}_{3}, 400 \mathrm{MHz}\right) \delta 8.36(1 \mathrm{H}, \mathrm{d}, J$ $=1.3 \mathrm{~Hz}), 7.95(1 \mathrm{H}, \mathrm{dd}, J=8.1,1.5 \mathrm{~Hz}), 7.92(1 \mathrm{H}, \mathrm{dd}, J=8.1,0.5 \mathrm{~Hz}), 5.02$ $(2 \mathrm{H}, \mathrm{s}) ;{ }^{13} \mathrm{C} \mathrm{NMR}\left(\mathrm{CDCl}_{3}, 100 \mathrm{MHz}\right) \delta 148.16,137.41,136.69,132.71,128.90,116.20$, 113.96, 42.21; GCMS (EI) m/z 161 (28\%) [M-Cl] ${ }^{+}, 151$ (8), 145 (11), 130 (20), 123 (28), 117 (100), 114 (81), 102 (35), 90 (74), 63 (47); HRMS (CI $\left.{ }^{+}\right) \mathrm{m} / z$ Calcd for $\mathrm{C}_{8} \mathrm{H}_{6} \mathrm{~N}_{2} \mathrm{O}_{2} \mathrm{Cl}[\mathrm{M}+\mathrm{H}]^{+}$: 197.0118; Found: 197.0116.

1-(Chloromethyl)-4-nitrobenzene $(2 \mathrm{j}) .^{3}$ Prepared from nitrobenzene following the General $\mathrm{NO}_{2}$ procedure B. Pale red solid (122 mg, 71\% yield); $R_{f} 0.29$ (cyclohexane/Et $\left.2 \mathrm{O}, 95: 5\right) ;{ }^{1} \mathrm{H}$ NMR $\left(\mathrm{CDCl}_{3}, 400 \mathrm{MHz}\right) \delta 8.22(1 \mathrm{H}, \mathrm{d}, J=8.8 \mathrm{~Hz}), 7.57(1 \mathrm{H}, \mathrm{d}, J=8.9 \mathrm{~Hz}), 4.65$ $(2 \mathrm{H}, \mathrm{s}) ;{ }^{13} \mathrm{C} \mathrm{NMR}\left(\mathrm{CDCl}_{3}, 100 \mathrm{MHz}\right) \delta 147.92,144.44,129.46,124.10,44.64$; GCMS (EI) $\mathrm{m} / z 173(14 \%)[\mathrm{M}]^{+}, 171(44)[\mathrm{M}]^{+}, 136$ (100), 96 (22), 52 (60), 51 (60), 50 (32), 30 (45); HRMS $\left(\mathrm{CI}^{+}\right) \mathrm{m} / z$ Calcd for $\mathrm{C}_{7} \mathrm{H}_{7} \mathrm{NO}_{2} \mathrm{Cl}[\mathrm{M}+\mathrm{H}]^{+}:$172.0165; Found: 172.0168.

2-(Chloromethyl)-1-nitronaphthalene $(2 \boldsymbol{k})$. Prepared from 1-nitronaphthalene following the<smiles>O=[N+]([O-])c1ccc2ccccc2c1CCl</smiles>

General procedure B. Beige solid (131 mg, $59 \%$ yield); m.p. $84-85{ }^{\circ} \mathrm{C} ; R_{f} 0.49$ (cyclohexane/Et $2 \mathrm{O}, 95: 5) ;{ }^{1} \mathrm{H} \mathrm{NMR}\left(\mathrm{CDCl}_{3}, 400 \mathrm{MHz}\right) \delta 8.02(1 \mathrm{H}, \mathrm{d}, J=8.5$ $\mathrm{Hz}), 7.93(1 \mathrm{H}, \mathrm{d}, J=7.8 \mathrm{~Hz}), 7.81(1 \mathrm{H}, \mathrm{d}, J=8.1 \mathrm{~Hz}), 7.69-7.62(3 \mathrm{H}, \mathrm{m}), 4.75(2 \mathrm{H}, \mathrm{s}) ;{ }^{13} \mathrm{C}$ $\mathrm{NMR}\left(\mathrm{CDCl}_{3}, 100 \mathrm{MHz}\right) \delta 147.38,133.75,131.61,129.22,128.23,128.16,126.95,126.45$, 124.452, 122.16, 41.08; GCMS (EI) m/z 223 (19\%) [M] $]^{+}, 221(55)[\mathrm{M}]^{+}, 186$ (16), 172 (24), 158 (31), 140 (55), 139 (100), 128 (63), 127 (42), 115 (44), 63 (18); HRMS (EI) m/z Calcd for $\mathrm{C}_{11} \mathrm{H}_{8} \mathrm{NO}_{2} \mathrm{Cl}[\mathrm{M}]^{+}:$221.0244; Found: 221.0242. Side products isolated: 4-(Chloromethyl)-1nitronaphthalene. Beige amorphous solid (42 mg, 19\% yield); $R_{f} 0.38$ (cyclohexane/Et $2 \mathrm{O}$, 95:5); ${ }^{1} \mathrm{H} \mathrm{NMR}\left(\mathrm{CDCl}_{3}, 400 \mathrm{MHz}\right) \delta 8.51(1 \mathrm{H}, \mathrm{m}), 8.24(1 \mathrm{H}, \mathrm{m}), 8.12(1 \mathrm{H}, \mathrm{d}, J=7.8 \mathrm{~Hz})$, $7.76(2 \mathrm{H}, \mathrm{m}), 7.63(1 \mathrm{H}, \mathrm{d}, J=7.8 \mathrm{~Hz}), 5.06(2 \mathrm{H}, \mathrm{s}) ;{ }^{13} \mathrm{C} \mathrm{NMR}\left(\mathrm{CDCl}_{3}, 100 \mathrm{MHz}\right) \delta 147.74$, 139.52 , 132.05, 129.46, 128.25, 125.93, 125.60, 124.32, 123.99, 123.06, 43.38; GCMS (EI) m/z $221(27 \%)[\mathrm{M}]^{+}, 186$ (24), 172 (28), 140 (34), 139 (100), 128 (80); HRMS (EI) m/z Calcd for $\mathrm{C}_{11} \mathrm{H}_{8} \mathrm{NO}_{2} \mathrm{Cl}[\mathrm{M}]^{+}$: 221.0244; Found: 221.0242; 2-(Dichloromethyl)naphthalene. Beige solid (26 mg, 21\% yield); m.p. 84-85 ${ }^{\circ} \mathrm{C} ; R_{f} 0.57$ (cyclohexane/Et $\left.2 \mathrm{O}, 95: 5\right) ;{ }^{1} \mathrm{H} \mathrm{NMR}\left(\mathrm{CDCl}_{3}\right.$, $400 \mathrm{MHz}) \delta 7.93(1 \mathrm{H}, \mathrm{s}), 7.92(1 \mathrm{H}, \mathrm{d}, J=9.3 \mathrm{~Hz}), 7.87(2 \mathrm{H}, \mathrm{m}), 7.75(1 \mathrm{H}, \mathrm{dd}, J=8.6,2.0$ $\mathrm{Hz}), 7.55(2 \mathrm{H}, \mathrm{m}), 6.89(1 \mathrm{H}, \mathrm{s}) ;{ }^{13} \mathrm{C} \mathrm{NMR}\left(\mathrm{CDCl}_{3}, 100 \mathrm{MHz}\right) \delta 137.56,133.92,132.55$, 129.36, 128.62, 127.93, 127.48, 127.04, 125.16, 123.65, 72.26; GCMS (EI) m/z 214 (2\%) $[\mathrm{M}]^{+}, 210(15)[\mathrm{M}]^{+}, 178$ (4), 177 (31), 175 (100), 140 (8), 139 (31), 115 (5), 98 (1), 87 (6), 
70 (6), 62 (3), 50 (2), 39 (1); HRMS (EI) $\mathrm{m} / z$ Calcd for $\mathrm{C}_{11} \mathrm{H}_{8} \mathrm{Cl}_{2}$ [M] : 210.0003; Found: 210.0001 .

2-(Chloromethyl)-4-methoxy-1-nitrobenzene $(2 l) .{ }^{4}$ Prepared from 1-methoxy-4-nitrobenene<smiles>COc1ccc([N+](=O)[O-])c(CCl)c1</smiles>
following the General procedure B. Yellow oil (192 mg, 95\% yield); $R_{f} 0.28$ (cyclohexane/Et $2 \mathrm{O}, 95: 5) ;{ }^{1} \mathrm{H} \mathrm{NMR}\left(\mathrm{CDCl}_{3}, 400 \mathrm{MHz}\right) \delta 8.13(1 \mathrm{H}, \mathrm{d}, J=9.1 \mathrm{~Hz})$, $7.17(1 \mathrm{H}, \mathrm{d}, J=2.8 \mathrm{~Hz}), 6.91(1 \mathrm{H}, \mathrm{dd}, J=9.1,2.8 \mathrm{~Hz}), 5.00(2 \mathrm{H}, \mathrm{s}), 3.91(3 \mathrm{H}, \mathrm{s})$; ${ }^{13} \mathrm{C} \mathrm{NMR}\left(\mathrm{CDCl}_{3}, 100 \mathrm{MHz}\right) \delta 163.80,140.85,135.60,128.30,116.62,113.81,56.17,43.82$; GCMS (EI) m/z 203 (4\%) [M] , 201 (11) [M] $]^{+}, 193$ (14), 184 (18), 166 (18), 123 (25), 106 (62), 73 (100), 63 (49); HRMS (EI) $m / z$ Calcd for $\mathrm{C}_{8} \mathrm{H}_{8} \mathrm{CINO}_{3}$ [M] $]^{+}$: 201.0193; Found: 201.0191.

1-(Chloromethyl)-4-methoxy-2-nitrobenzene $(\mathbf{2 m})$. Prepared from 1-methoxy-3-nitrobenene<smiles>COc1ccc(CCl)c([N+](=O)[O-])c1</smiles>
following the General procedure B (reaction time $24 \mathrm{~h}$ ). Yellow oil (173 mg, $86 \%$ yield); $R_{f} 0.21$ (cyclohexane/Et $\left.2 \mathrm{O}, 95: 5\right) ;{ }^{1} \mathrm{H} \mathrm{NMR}\left(\mathrm{CDCl}_{3}, 400 \mathrm{MHz}\right) \delta$ $7.53(1 \mathrm{H}, \mathrm{dd}, J=8.2,1.1 \mathrm{~Hz}), 7.45(1 \mathrm{H}, \mathrm{t}, J=8.3 \mathrm{~Hz}), 7.20-7.13(1 \mathrm{H}, \mathrm{m})$, $4.93(2 \mathrm{H}, \mathrm{s}), 3.97(3 \mathrm{H}, \mathrm{s}) ;{ }^{13} \mathrm{C} \mathrm{NMR}\left(\mathrm{CDCl}_{3}, 100 \mathrm{MHz}\right) \delta 158.38,130.21,121.03,116.82$, 115.77, 115.52, 56.85, 35.15; GCMS (EI) m/z 201 (20\%) [M] $]^{+}, 166$ (28), 149 (18), 138 (30), 125 (27), 107 (19), 92 (67), 77 (100), 51 (85); HRMS (EI) $m / z$ Calcd for $\mathrm{C}_{8} \mathrm{H}_{8} \mathrm{ClNO}_{3}[\mathrm{M}]^{+}$: 201.0193; Found: 201.0191.

1-Chloro-3-(chloromethyl)-4-methoxy-2-nitrobenzene (2n). Prepared from 1-chloro-4-

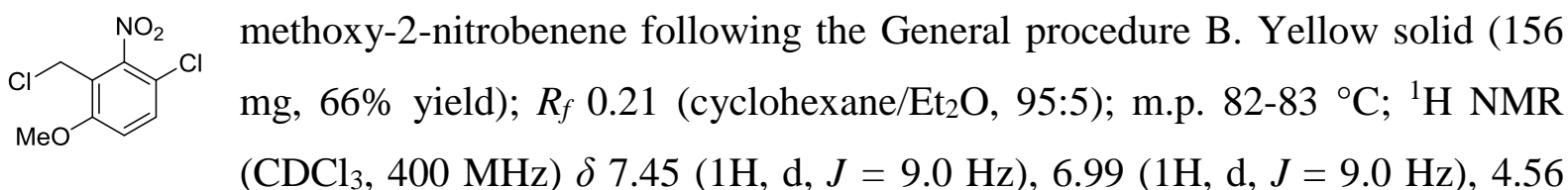
$(2 \mathrm{H}, \mathrm{s}), 3.95(3 \mathrm{H}, \mathrm{s}) ;{ }^{13} \mathrm{C} \mathrm{NMR}\left(\mathrm{CDCl}_{3}, 100 \mathrm{MHz}\right) \delta 156.68,131.58,120.06,116.52,113.71$, 56.86, 34.82; GCMS (EI) m/z 237 (27\%) [M] , 235 (41) [M] $]^{+}, 200$ (35), 183 (22), 159(24), 142 (42), 141 (64), 127 (48), 126 (53), 111 (61), 99 (35), 89 (39), 75 (100), 63 (31), 50 (36); HRMS (CI) $m / z$ Calcd for $\mathrm{C}_{8} \mathrm{H}_{7} \mathrm{Cl}_{2} \mathrm{NO}_{3}[\mathrm{M}]^{+}: 234.9803$; Found: 234.9798 .

2-(Chloromethyl)-4-(dimethoxymethyl)-1-nitrobenzene (2o). Prepared from 1-<smiles>COC(OC)c1ccc([N+](=O)[O-])c(CCl)c1</smiles(dimethoxymethyl)-4-nitrobenene following the General procedure B. Yellow oil (214 mg, 87\% yield); $R_{f} 0.14$ (cyclohexane/Et $\left.2 \mathrm{O}, 95: 5\right) ;{ }^{1} \mathrm{H} \mathrm{NMR}\left(\mathrm{CDCl}_{3}\right.$, $400 \mathrm{MHz}) \delta 8.06(1 \mathrm{H}, \mathrm{d}, J=8.4 \mathrm{~Hz}), 7.77(1 \mathrm{H}, \mathrm{d}, J=1.8 \mathrm{~Hz}), 7.59(1 \mathrm{H}, \mathrm{dd}, J$ $=8.4,1.8 \mathrm{~Hz}), 5.46(1 \mathrm{H}, \mathrm{s}), 4.98(2 \mathrm{H}, \mathrm{s}), 3.34(6 \mathrm{H}, \mathrm{s}) ;{ }^{13} \mathrm{C} \mathrm{NMR}\left(\mathrm{CDCl}_{3}, 100\right.$ MHz) $\delta 147.88,144.43,132.63,130.30,128.01$, 125.46, 101.40, 52.92, 42.94; GCMS (EI) m/z 216 (33\%) [M-OMe] ${ }^{+}, 214$ (100) [M-OMe] ${ }^{+}, 169$ (19), 153 (20), 89 (15), 75 (25); HRMS $\left(\mathrm{EI}^{+}\right) \mathrm{m} / z$ Calcd for $\mathrm{C}_{10} \mathrm{H}_{12} \mathrm{ClNO}_{4}[\mathrm{M}]^{+}:$245.0455; Found: 245.0459. 
1-Chloro-5-(chloromethyl)-2-(dimethoxymethyl)-4-nitrobenzene (2p). Prepared from 1-<smiles>COc1cc([N+](=O)[O-])c(CCl)cc1Cl</smiles>

chloro-2-(dimethoxymethyl)-4-nitrobenene following the General procedure B. Light yellow oil (232 mg, 83\% yield); $R_{f} 0.17$ (cyclohexane/Et $2 \mathrm{O}, 95: 5$ ) ${ }^{1} \mathrm{H}$ NMR $\left(\mathrm{CDCl}_{3}, 400 \mathrm{MHz}\right) \delta 8.35(1 \mathrm{H}, \mathrm{s}), 7.74(1 \mathrm{H}, \mathrm{s}), 5.61(1 \mathrm{H}, \mathrm{s}), 4.95$ $(2 \mathrm{H}, \mathrm{s}), 3.39(6 \mathrm{H}, \mathrm{s}) ;{ }^{13} \mathrm{C} \mathrm{NMR}\left(\mathrm{CDCl}_{3}, 100 \mathrm{MHz}\right) \delta 146.07,138.78,137.53,134.01,132.54$, 125.63, 99.59, 53.94 (2C), 42.23; GCMS (EI) m/z 281 (1\%) [M] $]^{+}, 252$ (11), 250 (64), 248 (100), 219 (5), 203 (10), 187 (7), 159 (11), 75 (18); HRMS (CI+) m/z Calcd for $\mathrm{C}_{10} \mathrm{H}_{12} \mathrm{NO}_{4} \mathrm{Cl}_{2}$ $[\mathrm{M}+\mathrm{H}]^{+}:$280.0143; Found: 280.0145 .

Methyl 3-(chloromethyl)-4-nitrobenzoate (2q). Prepared from 1-nitro-4-<smiles>COC(=O)c1ccc([N+](=O)[O-])c(CCl)c1</smiles(trimethoxymethyl)benene following the General procedure B. Beige amorphous solid (226 mg, 82\% yield); $R_{f} 0.17$ (cyclohexane/Et $\left.2 \mathrm{O}, 95: 5\right) ;{ }^{1} \mathrm{H} \mathrm{NMR}\left(\mathrm{CDCl}_{3}\right.$, $400 \mathrm{MHz}) \delta 8.35(1 \mathrm{H}, \mathrm{d}, J=1.8 \mathrm{~Hz}), 8.15(1 \mathrm{H}, \mathrm{dd}, J=8.5,1.8 \mathrm{~Hz}), 8.08(1 \mathrm{H}, \mathrm{d}$, $J=8.4 \mathrm{~Hz}), 4.97(2 \mathrm{H}, \mathrm{s}), 3.99(3 \mathrm{H}, \mathrm{s}) ;{ }^{13} \mathrm{C} \mathrm{NMR}\left(\mathrm{CDCl}_{3}, 100 \mathrm{MHz}\right) \delta 164.87,134.75$, 133.02, 132.81, 130.70, 125.48, 53.10, 42.31; GCMS (EI) m/z 194 (35\%) [M-Cl] $]^{+}, 166$ (65), 135 (100), 124 (28), 90 (19), 89 (66), 77 (30), 63 (51), 62 (23); HRMS (EI) m/z Calcd for $\mathrm{C}_{9} \mathrm{H}_{8} \mathrm{ClNO}_{4}[\mathrm{M}]^{+}:$229.0142; Found: 229.0148 .

2-(Chloromethyl)-4-(methoxymethoxy)-1-nitrobenzene (2r). Prepared from 1$\mathrm{NO}_{2}$ (methoxymethoxy)-4-nitrobenzene following the General procedure B. Light $\mathrm{Cl}_{\mathrm{Cl}}$ yellow oil (206 mg, 89\% yield); $R_{f} 0.11$ (pentane/ $\left.\mathrm{CH}_{2} \mathrm{Cl}_{2}, 80: 20\right) ;{ }^{1} \mathrm{H}$ NMR o $\mathrm{dd}, J=9.1,2.7 \mathrm{~Hz}), 5.27(2 \mathrm{H}, \mathrm{s}), 5.01(2 \mathrm{H}, \mathrm{s}) ; 3.50(3 \mathrm{H}, \mathrm{s}) ;{ }^{13} \mathrm{C} \mathrm{NMR}\left(\mathrm{CDCl}_{3}, 100 \mathrm{MHz}\right) \delta$ 161.42, 141.66, 135.47, 128.13, 118.13, 115.86, 94.53, 56.71, 43.62; GCMS (EI) m/z 233 (31\%) [M] $]^{+}, 231$ (100) [M] $]^{+}, 197$ (2), 196 (11), 125 (3), 123 (3), 89 (11); HRMS (EI) m/z Calcd for $\mathrm{C}_{9} \mathrm{H}_{10} \mathrm{ClNO}_{4}[\mathrm{M}]^{+}:$231.0298; Found: 231.0297.

3-(Chloromethyl)-2-nitrofuran (2s). Prepared from 2-nitrofuran following the General Cl procedure B. Yellow oil (91 mg, 56\% yield); $R_{f} 0.15$ (cyclohexane/Et $\left.{ }_{2} \mathrm{O}, 95: 5\right) ;{ }^{1} \mathrm{H}$ NMR $\left(\mathrm{CDCl}_{3}, 400 \mathrm{MHz}\right) \delta 7.27(1 \mathrm{H}, \mathrm{d}, J=3.7 \mathrm{~Hz}), 6.62(1 \mathrm{H}, \mathrm{dt}, J=3.7,0.7 \mathrm{~Hz})$, $4.59(2 \mathrm{H}, \mathrm{s}) ;{ }^{13} \mathrm{C} \mathrm{NMR}\left(\mathrm{CDCl}_{3}, 100 \mathrm{MHz}\right) \delta 153.33,152.18,112.51,112.27,36.20$; GCMS (EI) m/z 163 (11\%) [M] $]^{+}, 161$ (33) [M]+, 126 (100), 96 (22), 52 (60), 51 (60), 50 (32), 30 (45); HRMS (EI $\left.{ }^{+}\right) \mathrm{m} / z$ Calcd for $\mathrm{C}_{5} \mathrm{H}_{4} \mathrm{NO}_{3} \mathrm{Cl}[\mathrm{M}]^{+}:$: 160.9880; Found: 160.9879.

3-(Chloromethyl)-2-nitrothiophene (2t). Prepared from 2-nitrothiophenen following the Cl General procedure B. Yellow oil (100 mg, 56\% yield); $R_{f} 0.24$ (cyclohexane/Et $2 \mathrm{O}$, 95:5); ${ }^{1} \mathrm{H} \mathrm{NMR}\left(\mathrm{CDCl}_{3}, 400 \mathrm{MHz}\right) \delta 7.79(1 \mathrm{H}, \mathrm{d}, J=4.2 \mathrm{~Hz}), 7.04(1 \mathrm{H}, \mathrm{dt}, J=4.2$, 
$0.8 \mathrm{~Hz}), 4.73(2 \mathrm{H}, \mathrm{s}) ;{ }^{13} \mathrm{C} \mathrm{NMR}\left(\mathrm{CDCl}_{3}, 100 \mathrm{MHz}\right) \delta 152.02,148.12,128.41,126.58,39.59$; GCMS (EI) m/z 179 (12\%) [M] $]^{+}, 177$ (34) [M] , 142 (100), 112 (7), 96 (25), 84 (12), 69 (19), 45 (12); HRMS (EI) $m / z$ Calcd for $\mathrm{C}_{5} \mathrm{H}_{4} \mathrm{SCINO}_{2}[\mathrm{M}]^{+}$: 176.9651 ; Found: 176.9653.

SNAr reactions with substituted nitrobenzenes. Following the General procedure A, 1,4dinitrobenzene afforded 1-(dichloromethyl)-4-nitrobenzene (3a) in $89 \%$ yield; ${ }^{1} \mathrm{H}$ NMR $\left(\mathrm{CDCl}_{3}, 400 \mathrm{MHz}\right) \delta 8.27-8.30(\mathrm{~m}, 2 \mathrm{H}), 7.75-7.79(\mathrm{~m}, 2 \mathrm{H}), 6.76(\mathrm{~s}, 1 \mathrm{H}) ;{ }^{13} \mathrm{C} \mathrm{NMR}\left(\mathrm{CDCl}_{3}\right.$, 100.6 Hz) $\delta$ 148.7, 146.4, 127.5, 124.3, 69.9; GCMS (EI) m/z $207(7 \%)[\mathrm{M}]^{+}, 205(8)[\mathrm{M}]^{+}$, $172(33)[\mathrm{M}-\mathrm{Cl}]^{+}, 170$ (100) [M - Cl] $]^{+}, 159$ (4), 140 (15), 126 (4), 124 (13), 89 (55), 77 (10); 1,2-dinitrobenzene afforded 1-(dichloromethyl)-2-nitrobenzene (3b) $73 \%$ yield; ${ }^{1} \mathrm{H}$ NMR $\left(\mathrm{CDCl}_{3}, 400 \mathrm{MHz}\right) \delta 8.20(\mathrm{dd}, J=8.0,1.4 \mathrm{~Hz}, 1 \mathrm{H}), 8.00(\mathrm{dd}, J=8.3,1.4 \mathrm{~Hz}, 1 \mathrm{H}), 7.75-7.79$ $(\mathrm{m}, 1 \mathrm{H}), 7.55-7.59(\mathrm{~m}, 2 \mathrm{H}) ;{ }^{13} \mathrm{C} \mathrm{NMR}\left(\mathrm{CDCl}_{3}, 100.6 \mathrm{~Hz}\right) \delta 135.0,134.3,130.8,130.1,124.8$, 66.0; GCMS (EI) m/z $207(11 \%)[\mathrm{M}]^{+}, 205(6)[\mathrm{M}]^{+}, 172(29)[\mathrm{M}-\mathrm{Cl}]^{+}, 170(100)[\mathrm{M}-\mathrm{Cl}]^{+}$, 159 (7), 139 (10), 126(7), 124 (23), 89 (50), 77 (14); 1-fluoro-4-nitrobezene afforded 3a in 99\% GCMS conversion; 1-chloro-4-nitrobezene afforded 3a in 98\% GCMS conversion and 1bromo-4-nitrobezene afforded 3a in 99\% GCMS conversion.

\section{Synthesis of compounds 4 by a one-pot chloromethylation/alkylation sequence; General}

procedure C. A solution of $n$-BuLi (2.5 M, $1.6 \mathrm{~mL}, 4 \mathrm{mmol}, 4$ equiv.) in hexanes was added over 5 min to a solution of $\mathrm{CH}_{2} \mathrm{Cl}_{2}$ (4 mmol, 4 equiv.) in dry THF ( $8 \mathrm{~mL}$ ) cooled to $-100{ }^{\circ} \mathrm{C}$ under argon. After stirring the mixture for $15 \mathrm{~min}$ the resulting solution was added via syringe to a solution of 4-nitro-1-(pentafluorosulfanyl)benzene (1a) (249 mg, 1 mmol, 1 equiv.) in dry THF ( $8 \mathrm{~mL}$ ) cooled to $-100{ }^{\circ} \mathrm{C}$ (liquid $\mathrm{N}_{2} / \mathrm{EtOH}$ ) under argon. The resulting mixture was stirred at this temperature for $30 \mathrm{~s}$, followed by the addition of cold $\left(0{ }^{\circ} \mathrm{C}\right)$ conc. aqueous $\mathrm{HCl}$ ( $0.45 \mathrm{~mL}, 4.5 \mathrm{mmol}, 4.5$ equiv.). The cooling bath was removed and the mixture was slowly warmed to rt. Then the reaction mixture was diluted with EtOH $(10 \mathrm{~mL})$ and a mixture of nucleophile ( 3 mmol, 3 equiv) and $\mathrm{Et}_{3} \mathrm{~N}(0.42 \mathrm{~mL}, 3 \mathrm{mmol}, 3$ equiv) in $\mathrm{EtOH}(5 \mathrm{~mL})$ was added over $10 \mathrm{~min}$. The reaction was monitored using TLC (to complete disappearance of $\mathbf{2 a}$ ). A mixture of water and brine $(1>1,10 \mathrm{~mL})$ was added, the product was extracted with $\mathrm{Et}_{2} \mathrm{O}$, the combined organic phase was washed with water, brine, dried $\left(\mathrm{MgSO}_{4}\right)$ and solvent was removed under reduced pressure. Purification using flash chromatography (silica gel, hexane/ $\mathrm{CH}_{2} \mathrm{Cl}_{2}$ ) provided pure product 4 . 
4-(2-Nitro-5-(pentafluorosulfanyl)benzyl)morpholine (4a). Prepared from morpholine following the General procedure $\mathrm{C}$ (reaction time $16 \mathrm{~h})$. Yellow oil $(160 \mathrm{mg}$,
$46 \%$ yield); $R_{f} 0.11$ (cyclohexane/Et $\left.2 \mathrm{O}, 95: 5\right) ;{ }^{1} \mathrm{H} \mathrm{NMR}\left(\mathrm{CDCl}_{3}, 400 \mathrm{MHz}\right) \delta$ $\mathrm{Hz}), 3.81(2 \mathrm{H}, \mathrm{s}), 3.70-3.65(4 \mathrm{H}, \mathrm{m}), 2.48-2.44(4 \mathrm{H}, \mathrm{m}) ;{ }^{13} \mathrm{C} \mathrm{NMR}\left(\mathrm{CDCl}_{3}, 100 \mathrm{MHz}\right) \delta$ 155.95 (quint, $J=18.7 \mathrm{~Hz}$ ), 150.94, 135.21, 128.82 (quint, $J=4.6 \mathrm{~Hz}$ ), 126.20 (quint, $J=4.5$ $\mathrm{Hz}), 124.97,66.95$ (2C), 59.11, $53.58(2 \mathrm{C}) ;{ }^{19} \mathrm{~F} \mathrm{NMR}\left(\mathrm{CDCl}_{3}, 376 \mathrm{MHz}\right) \delta 81.15(1 \mathrm{~F}, \mathrm{~m})$, 62.01 (4F, d, $J=150.6 \mathrm{~Hz}$ ); GCMS (EI) m/z 348 (5\%) [M] $]^{+}, 331$ (100), 300 (91), 272 (23), 258 (13), 246 (30), 221 (11), 174 (5), 135 (22), 116 (30), 109 (44), 89 (60), 63 (32), 56 (44), 42 (23); HRMS (EI) $m / z$ Calcd for $\mathrm{C}_{11} \mathrm{H}_{13} \mathrm{~N}_{2} \mathrm{O}_{3} \mathrm{SF}_{5}[\mathrm{M}]^{+}: 348.0567$; Found: 348.0571.

2,4-Dimethoxy-N-(2-nitro-5-(pentafluorosulfanyl)benzyl)aniline (4b). Prepared from 2-4-

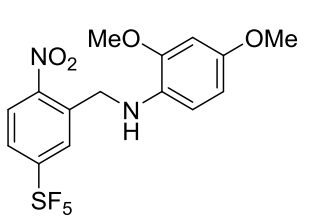

dimethoxyaniline following the General procedure $\mathrm{C}$ (reaction time $16 \mathrm{~h}$ ). Orange amorphous solid (167 mg, $40 \%$ yield); $R_{f} 0.17$ (cyclohexane/Et $2 \mathrm{O}$, 95:5); ${ }^{1} \mathrm{H} \mathrm{NMR}\left(\mathrm{CDCl}_{3}, 400 \mathrm{MHz}\right) \delta 8.10(2 \mathrm{H}, \mathrm{dd}, J=3.1,2.5 \mathrm{~Hz}), 7.80$ $(1 \mathrm{H}, \mathrm{dd}, J=8.9,2.5 \mathrm{~Hz}), 6.48(1 \mathrm{H}, \mathrm{d}, J=2.4 \mathrm{~Hz}), 6.31(1 \mathrm{H}, \mathrm{dd}, J=8.6,2.4 \mathrm{~Hz}), 6.28(1 \mathrm{H}$, $\mathrm{dd}, J=8.6,0.5 \mathrm{~Hz}), 4.71(2 \mathrm{H}, \mathrm{s}), 4.59(1 \mathrm{H}$, br s$), 3.87(3 \mathrm{H}, \mathrm{s}), 3.73(3 \mathrm{H}, \mathrm{s}) ;{ }^{13} \mathrm{C} \mathrm{NMR}$ $\left(\mathrm{CDCl}_{3}, 100 \mathrm{MHz}\right) \delta 153.12,149.44,148.39,138.12,131.09,128.12(\mathrm{~m}), 125.90(\mathrm{~m}), 125.52$, 111.34, 103.96, 99.54, 55.86, 55.74, 46.82; ${ }^{19} \mathrm{~F} \mathrm{NMR}\left(\mathrm{CDCl}_{3}, 376 \mathrm{MHz}\right) \delta 81.83-81.07(1 \mathrm{~F}$, m), 63.45 (4F, d, $J=150.8 \mathrm{~Hz}$ ); GCMS (EI) m/z 414 (100\%) [M] $]^{+}, 399$ (6), 366 (8), 336 (10), 287 (13), 262 (10), 153 (19), 151 (66), 138 (42), 124 (28), 93 (24), 63 (15), 39 (7); HRMS (EI) $m / z$ Calcd for $\mathrm{C}_{15} \mathrm{H}_{15} \mathrm{~N}_{2} \mathrm{O}_{4} \mathrm{SF}_{5}[\mathrm{M}]^{+}:$414.0673; Found: 414.0669.

Pentafluoro(4-nitro-3-((phenylthio)methyl)phenyl)- $\lambda^{6}$-sulfane (4c). Prepared from thiophenol

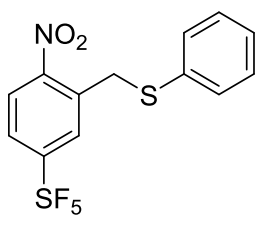

following the General procedure C (reaction time $1 \mathrm{~h}$ ). Yellow oil (189 mg, $51 \%$ yield); $R_{f} 0.58$ (cyclohexane/Et ${ }_{2} \mathrm{O}, 95: 5$ ); ${ }^{1} \mathrm{H}$ NMR (acetone- $d_{6}, 400$ MHz) $\delta 8.20(1 \mathrm{H}, \mathrm{d}, J=8.9 \mathrm{~Hz}), 8.02(1 \mathrm{H}, \mathrm{dd}, J=8.9,2.5 \mathrm{~Hz}), 7.82(1 \mathrm{H}, \mathrm{d}$, $J=2.5 \mathrm{~Hz}), 7.31(5 \mathrm{H}, \mathrm{m}), 4.59(2 \mathrm{H}, \mathrm{s}) ;{ }^{13} \mathrm{C}$ NMR (acetone- $\left.d_{6}, 100 \mathrm{MHz}\right) \delta$ 151.27, 136.00, 134.38, 133.18(2C), 130.67 (quint, $J=4.8 \mathrm{~Hz}), 130.16$ (2C), 128.89, 127.39 (quint, $J=4.7 \mathrm{~Hz}$ ), 127.16, 125.08, 36.97; ${ }^{19} \mathrm{~F}$ NMR (acetone- $\left.d_{6}, 376 \mathrm{MHz}\right) \delta 82.45(1 \mathrm{~F}, \mathrm{~m}$ ), 62.96 (4F, d, $J=149.4 \mathrm{~Hz}$ ); GCMS (EI) m/z 371 (19\%) [M] $]^{+}, 306$ (7), 246 (30), 126 (26), 110 (100), 109 (63), 108 (11), 65 (25); HRMS (EI) $\mathrm{m} / z$ Calcd for $\mathrm{C}_{13} \mathrm{H}_{10} \mathrm{~F}_{5} \mathrm{NO}_{2} \mathrm{~S}_{2}[\mathrm{M}]^{+}:$: 371.0073 ; Found: 371.0071.

\section{Synthesis of compounds 5 by a one-pot chloromethylation/oxidation sequence; General}

procedure D. A solution of $n$-BuLi (2.5 M, $1.6 \mathrm{~mL}, 4 \mathrm{mmol}, 4$ equiv.) in hexanes was added 
over 5 min to a solution of $\mathrm{CH}_{2} \mathrm{Cl}_{2}$ (4 mmol, 4 equiv.) in dry THF (8 mL) cooled to $-100{ }^{\circ} \mathrm{C}$ under argon. After stirring the mixture for $15 \mathrm{~min}$ the resulting solution was added via syringe to a solution of substituted nitrobenzene 1 (1 mmol, 1 equiv.) in dry THF ( $8 \mathrm{~mL})$ cooled to $100{ }^{\circ} \mathrm{C}$ (liquid $\mathrm{N}_{2} / \mathrm{EtOH}$ ) under argon. The resulting mixture was stirred at this temperature for $30 \mathrm{~s}$, followed by the addition of cold $\left(0{ }^{\circ} \mathrm{C}\right)$ conc. aqueous $\mathrm{HCl}(0.45 \mathrm{~mL}, 4.5 \mathrm{mmol}, 4.5$ equiv.). The cooling bath was removed and the mixture was slowly warmed to rt. The reaction mixture was diluted with $\mathrm{EtOH}(10 \mathrm{~mL})$ and a mixture of $\mathrm{Et}_{3} \mathrm{~N}(0.7 \mathrm{~mL}, 5 \mathrm{mmol}, 5$ equiv) in EtOH $(25 \mathrm{~mL})$ added over $10 \mathrm{~min}$. The mixture was stirred at $\mathrm{rt}$ in open flask and monitored by TLC for complete disappearance of $\mathbf{1}$. Charcoal $(\sim 0.1 \mathrm{~g})$ was added and the stirring continued for $1 \mathrm{~h}$, followed by filtration. A mixture of water and brine $(1: 1,10 \mathrm{~mL})$ was added, the product was extracted with $\mathrm{Et}_{2} \mathrm{O}$, the combined organic phase was washed with water, brine, dried $\left(\mathrm{MgSO}_{4}\right)$ and solvent was removed under reduced pressure. Purification using flash chromatography (silica gel, hexane $/ \mathrm{CH}_{2} \mathrm{Cl}_{2}$ ) provided pure product 5 .

2,4-Dinitrobenzaldehyde (5a). Prepared from 1,3-dinitrobenzene (1h) following the General<smiles>O=Cc1ccc([N+](=O)[O-])cc1[N+](=O)[O-]</smiles>
procedure D (reaction time for oxidation $48 \mathrm{~h}$ ). Brown solid (116 mg, 68\% yield); $R_{f} 0.56$ (pentane/EtOAc, $\left.75: 25\right) ;$ m.p. $68-69{ }^{\circ} \mathrm{C} ;{ }^{1} \mathrm{H} \mathrm{NMR}\left(\mathrm{CDCl}_{3}, 400\right.$ MHz) $\delta 10.51(1 \mathrm{H}, \mathrm{s}), 9.00(1 \mathrm{H}, \mathrm{d}, J=2.0 \mathrm{~Hz}), 8.72(1 \mathrm{H}, \mathrm{dd}, J=3.2,2.0$ $\mathrm{Hz}), 8.21(1 \mathrm{H}, \mathrm{d}, J=3.2 \mathrm{~Hz}) ;{ }^{13} \mathrm{C} \mathrm{NMR}\left(\mathrm{CDCl}_{3}, 100 \mathrm{MHz}\right) \delta 186.70,150.11,149.51$, 135.62, 131.64, 128.63, 120.28; GCMS (EI) m/z 196 (1\%) [M] ${ }^{+}, 166$ (1), 149 (7), 120 (100), 108 (60), 103 (29), 75 (91), 50 (58); HRMS (CI) m/z Calcd for $\mathrm{C}_{7} \mathrm{H}_{4} \mathrm{~N}_{2} \mathrm{O}_{5}[\mathrm{M}]^{+}:$196.0120; Found: 196.0123.

4-Formyl-3-nitrobenzonitrile $(\mathbf{5 b}) .^{5}$ Prepared from 3-nitrobenzonitrile (1j) following the CHO General procedure D (reaction time for oxidation $48 \mathrm{~h}$ ). Beige solid (116 mg, $66 \%$ yield); $R_{f} 0.66$ (pentane/EtOAc, 75:25); m.p. 112-113 ${ }^{\circ} \mathrm{C} ;{ }^{1} \mathrm{H}$ NMR $\left(\mathrm{CDCl}_{3}, 400 \mathrm{MHz}\right) \delta 10.47(1 \mathrm{H}, \mathrm{s}), 8.44(1 \mathrm{H}, \mathrm{t}, J=1.0 \mathrm{~Hz}), 8.04(2 \mathrm{H}, \mathrm{d}, J=$ $1.0 \mathrm{~Hz}) ;{ }^{13} \mathrm{C} \mathrm{NMR}\left(\mathrm{CDCl}_{3}, 100 \mathrm{MHz}\right) \delta 186.50,137.37,134.27,130.96,128.34,117.73$, 115.78; GCMS (EI) m/z, 177 (2\%) [M+H] ${ }^{+}, 162$ (5), 144 (60), 118 (76), 116 (31), 89 (21), 64 (32), 63 (32); HRMS (CI) $m / z$ Calcd for $\mathrm{C}_{8} \mathrm{H}_{5} \mathrm{~N}_{2} \mathrm{O}_{3}[\mathrm{M}+\mathrm{H}]^{+}:$177.0300; Found: 177.0303.

4-Nitro-3-(trifluoromethyl)benzaldehyde $\quad(\mathbf{5 c})$ Prepared $\quad$ from 1-nitro-2(trifluoromethyl)benzene $(\mathbf{I g})$ following the General procedure D (reaction time
for oxidation 13 days). Yellow oil $\left(112 \mathrm{mg}, 51 \%\right.$ yield) $R_{f} 0.18$ (pentane/Et $2 \mathrm{O}$,
$75: 25) ;{ }^{1} \mathrm{H} \mathrm{NMR}\left(\mathrm{CDCl}_{3}, 400 \mathrm{MHz}\right) \delta 10.16(1 \mathrm{H}, \mathrm{s}), 8.34(1 \mathrm{H}, \mathrm{d}, J=1.6 \mathrm{~Hz})$,
$8.25(1 \mathrm{H}, \mathrm{dd}, J=8.2,1.7 \mathrm{~Hz}), 8.03(1 \mathrm{H}, \mathrm{d}, J=8.2 \mathrm{~Hz}) ;{ }^{13} \mathrm{C} \mathrm{NMR}\left(\mathrm{CDCl}_{3}, 100\right.$ $\mathrm{MHz}) \delta 188.64,138.51,134.02,129.11(\mathrm{q}, J=17.8 \mathrm{~Hz}), 126.00 ;{ }^{19} \mathrm{~F}$ NMR $\left(\mathrm{CDCl}_{3}, 376\right.$ 
MHz) $\delta-60.81$ (3F, s); GCMS (EI) m/z, 219 (100\%) [M]+, 218 (54), 200 (13), 173 (63), 172 (30), 160 (22), 125 (52), 95 (48), 75 (80); HRMS (CI) m/z Calcd for $\mathrm{C}_{8} \mathrm{H}_{5} \mathrm{NO}_{3} \mathrm{~F}_{3}[\mathrm{M}+\mathrm{H}]^{+}$: 220.0222; Found: 220.0220.

\section{Crystallographic data}

Single-crystal diffraction data of $\mathbf{2 k}$ were collected on Xcalibur PX diffractometr with monochromatized $\mathrm{Cu}_{\mathrm{K} \alpha}$ radiation $(\lambda=1.54180 \AA)$ at $180 \mathrm{~K}$. CrysAlisProCCD ${ }^{6}$ was used for data collection, cell refinement and data reduction. The structure was solved by direct methods with SIR92 ${ }^{7}$ and refined by full-matrix least-squares on F with CRYSTALS. ${ }^{8}$ The positional and anisotropical thermal parameters of all non-hydrogen atoms were refined. All hydrogen atoms were located in a difference Fourier map. Then they were repositioned geometrically and were refined with riding constraints.

Crystal data for $2 \boldsymbol{k}(0.11 \times 0.17 \times 0.41 \mathrm{~mm}): \mathrm{C}_{11} \mathrm{H}_{8} \mathrm{Cl}_{1} \mathrm{~N}_{1} \mathrm{O}_{2}$, orthorhombic, space group Pbca, $a=12.8621(5) \AA, b=7.5303(3) \AA, c=20.7636(8) \AA, V=2011.07(14) \AA^{3}, Z=8, M=221.64$, 14070 reflections measured, 1830 independent reflections. Final $R=0.065, w R=0.069$, $G o F=0.957$ for 1707 reflections with $I>2 \sigma(I)$ and 137 parameters. CCDC 1906014 .

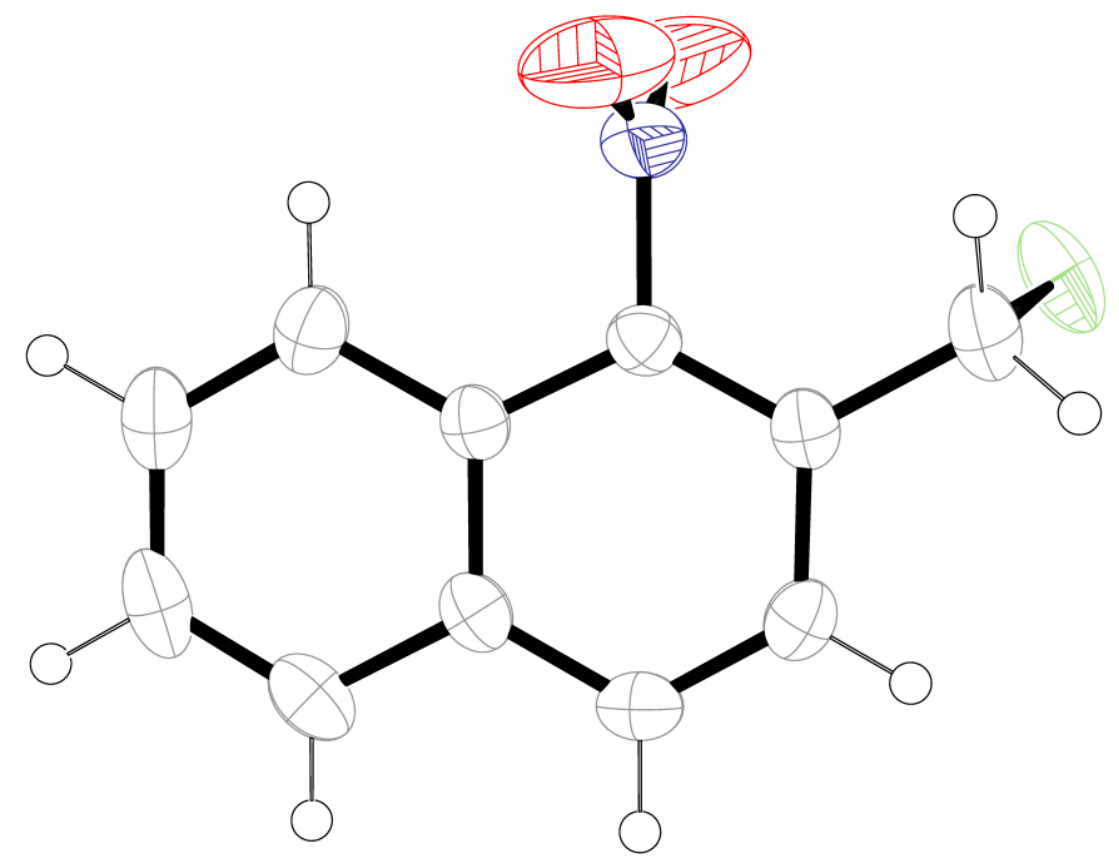

Figure 1: ORTEP ${ }^{9}$ diagram of $\mathbf{2 k}$, displacement ellipsoids shown with $50 \%$ probability. 


\section{References}

1. X. Chen, Y. Yu, Z. Liao, H. Li, W. Wang Tetrahedron Lett. 2016, 57, 5742-5745.

2. L. A. Berven, D. Dolphin, S. G. Withers Can. J. Chem. 1990, 68, 1859-1866.

3. Z. Wang, L. Zhu, F. Yin, Z. Su, Z. Li J. Am. Chem. Soc. 2012, 134, 4258-4263.

4. M. Tercel, W. R. Wilson, R. F. Anderson, W. A. Denny J. Med. Chem. 1996, 39, 1084-1094.

5. Y. Ji, D. A. DiRocco, C. M. Hong, M. K. Wismer, M. Reibarkh Org. Lett. 2018, 20, 2156-2159.

6. CrysAlisPro, Oxford Diffraction, 2002.

7. A. Altomare, G. Cascarano, G. Giacovazzo, A. Guagliardi, M. C. Burla, G. Polidori, M. Camalli J. Appl. Cryst. 1994, 27, 435.

8. P. W. Betteridge, J. R. Carruthers, R. I. Cooper, K. Prout, D. J. Watkin J. Appl. Cryst. 2003, 36, 1487.

9. L. J. Farrugia J. Appl. Cryst. 2012, 45, 849-854. 


\section{Copies of NMR spectra}

2a, ${ }^{1} \mathrm{H} \mathrm{NMR}\left(\mathrm{CDCl}_{3}, 400 \mathrm{MHz}\right)$

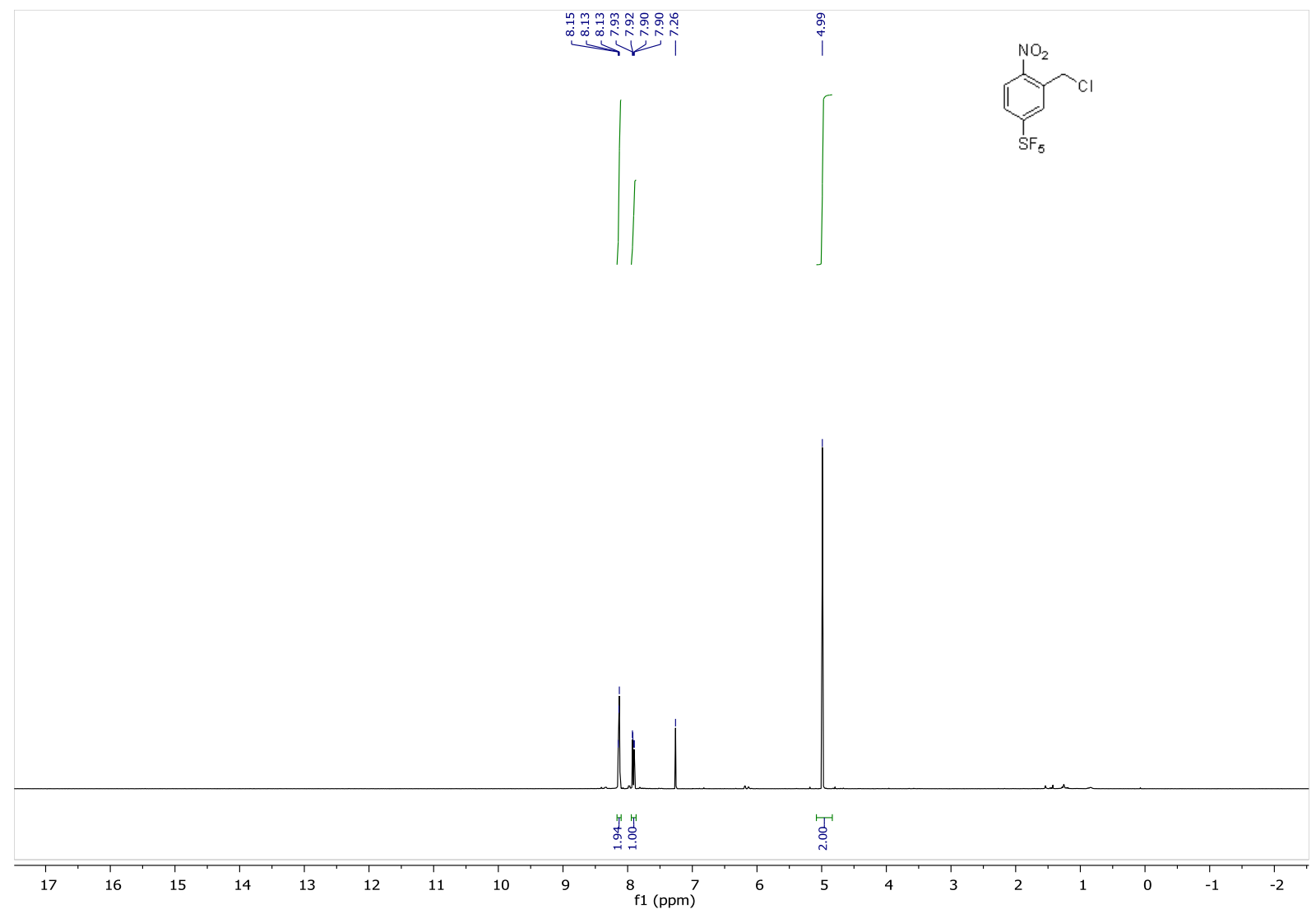

2a, ${ }^{13} \mathrm{C} \mathrm{NMR}\left(\mathrm{CDCl}_{3}, 100 \mathrm{MHz}\right)$<smiles>O=C(O)C1CCCCC1</smiles> 
2a, ${ }^{19} \mathrm{~F}$ NMR $\left(\mathrm{CDCl}_{3}, 376 \mathrm{MHz}\right)$

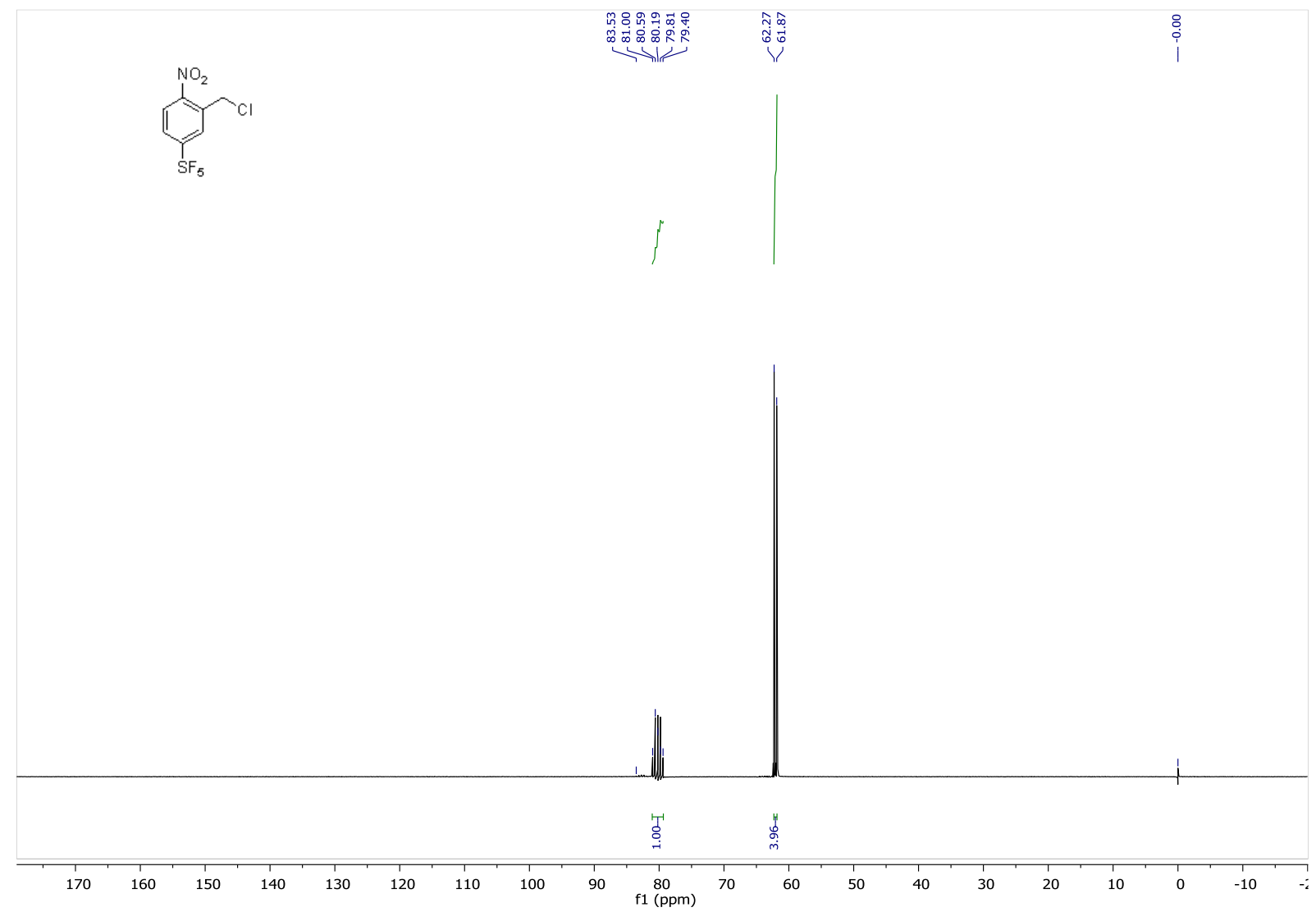

2b, ${ }^{1} \mathrm{H} \mathrm{NMR}\left(\mathrm{CDCl}_{3}, 400 \mathrm{MHz}\right)$

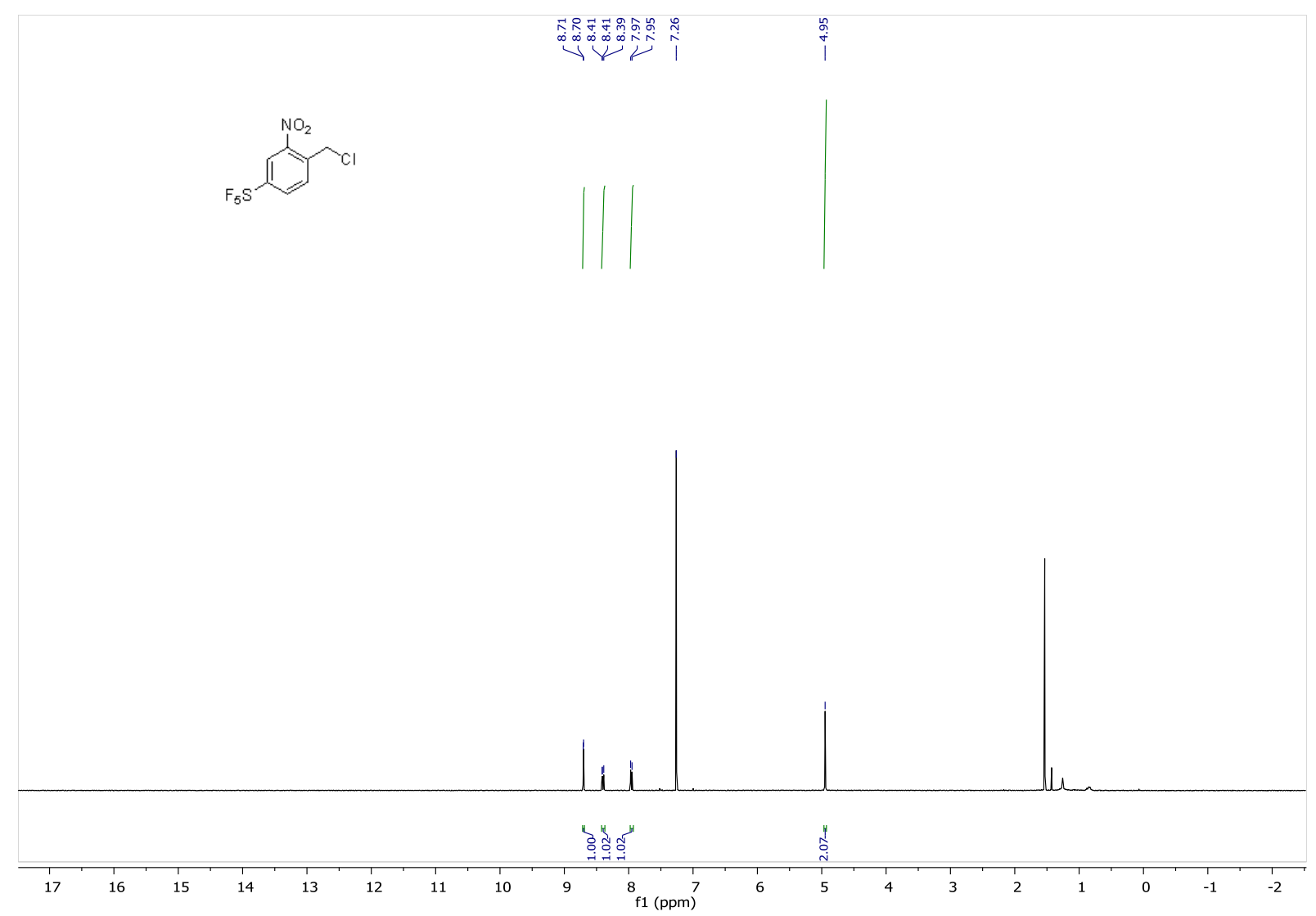

2b, ${ }^{13} \mathrm{C} \mathrm{NMR}\left(\mathrm{CDCl}_{3}, 100 \mathrm{MHz}\right)$ 


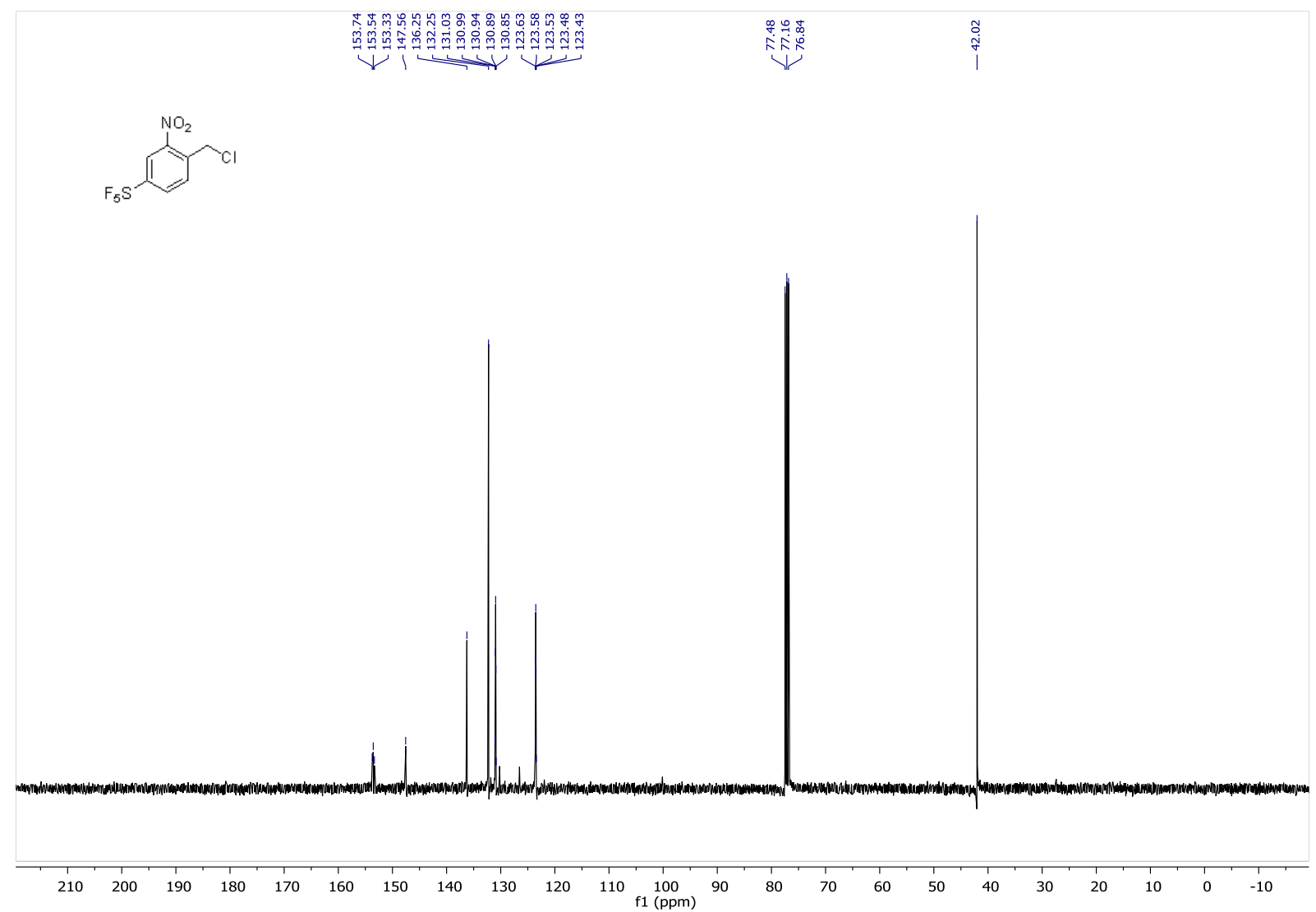

2b, ${ }^{19} \mathrm{~F} \mathrm{NMR}\left(\mathrm{CDCl}_{3}, 376 \mathrm{MHz}\right)$

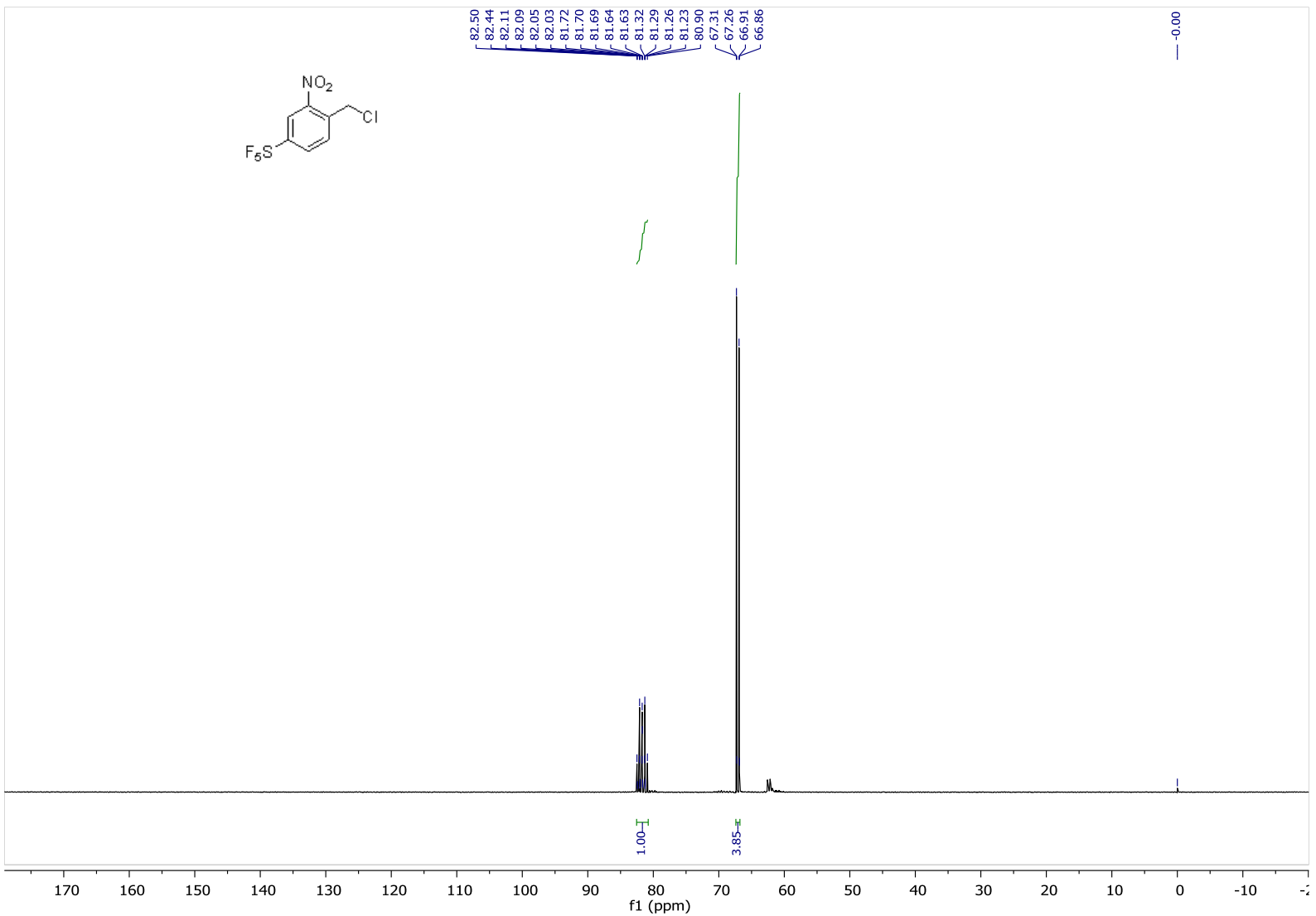

2c, ${ }^{1} \mathrm{H} \mathrm{NMR}\left(\mathrm{CDCl}_{3}, 400 \mathrm{MHz}\right)$ 


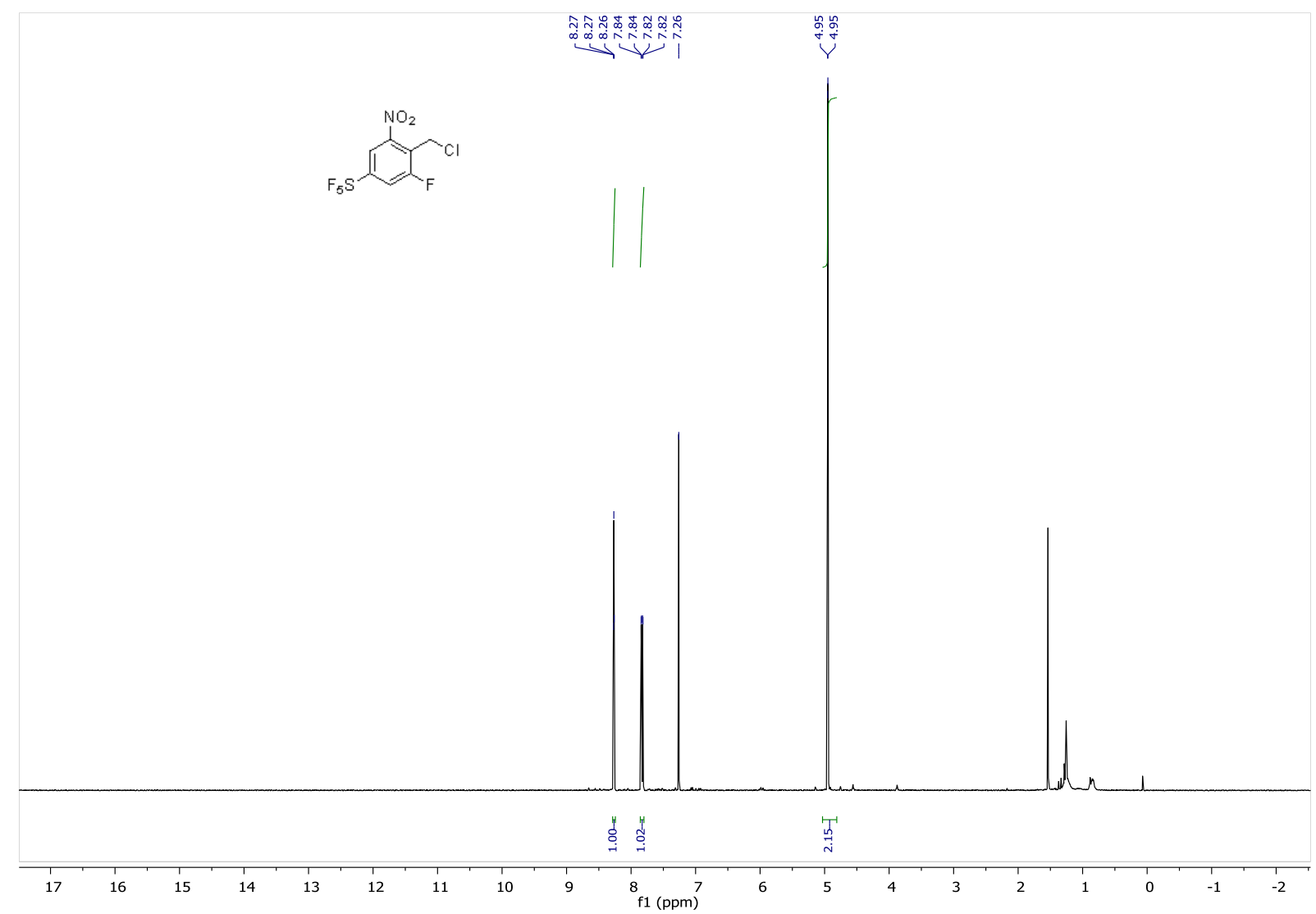

2c, ${ }^{13} \mathrm{C}$ NMR (APT, $\left.\mathrm{CDCl}_{3}, 100 \mathrm{MHz}\right)$

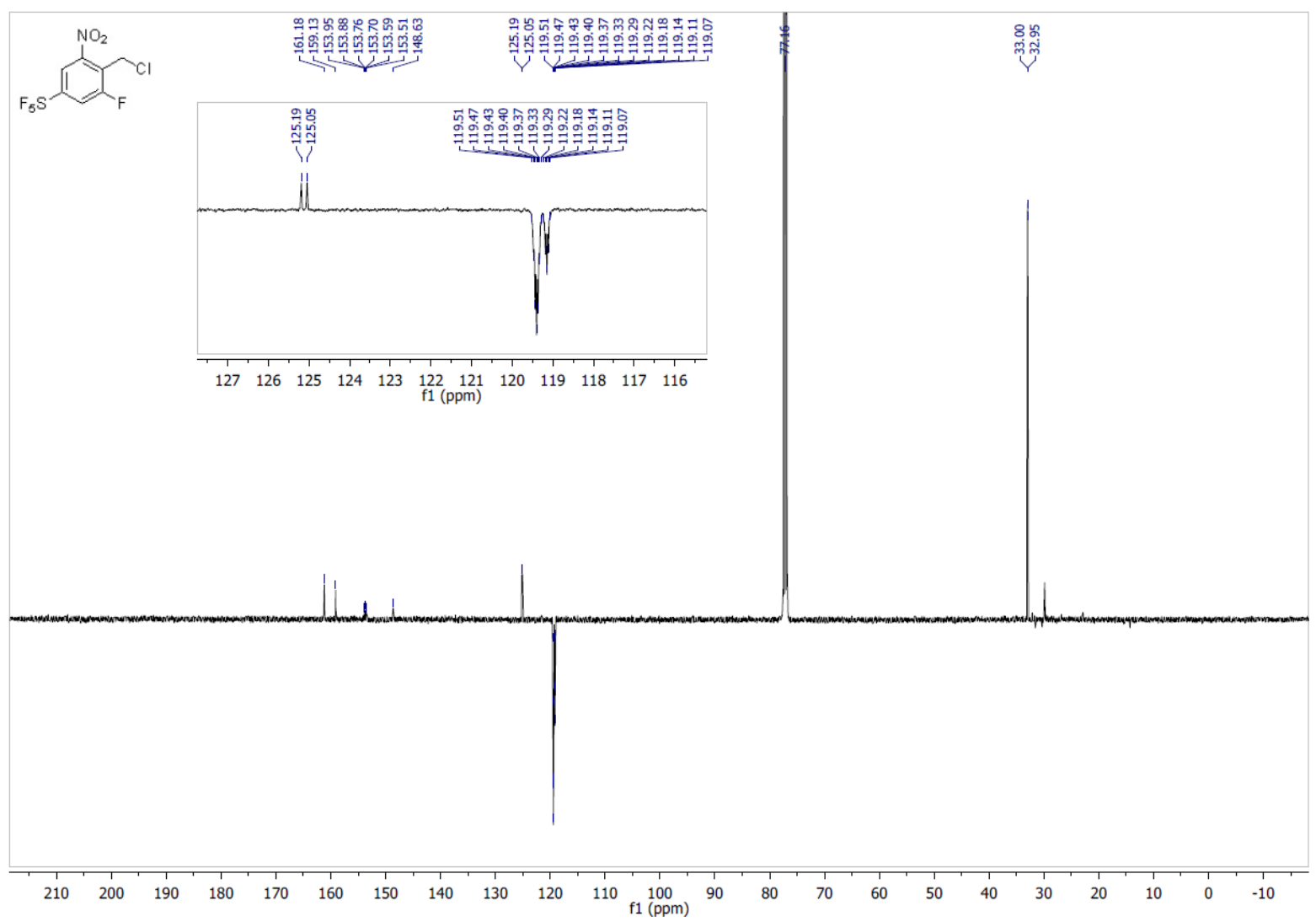

2c, ${ }^{19} \mathrm{~F}$ NMR $\left(\mathrm{CDCl}_{3}, 376 \mathrm{MHz}\right)$ 


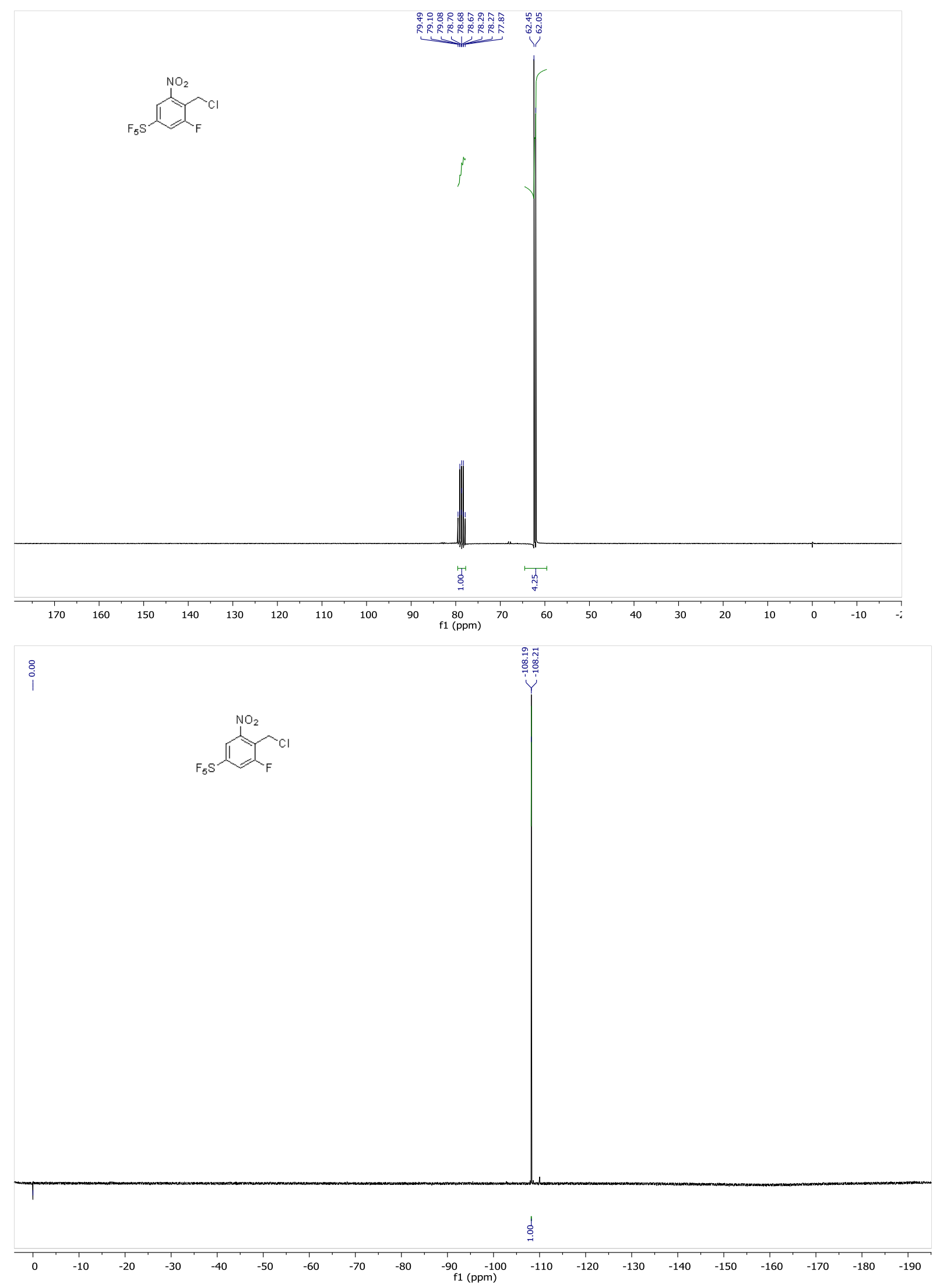

2d, ${ }^{1} \mathrm{H} \mathrm{NMR}\left(\mathrm{CDCl}_{3}, 400 \mathrm{MHz}\right)$ 


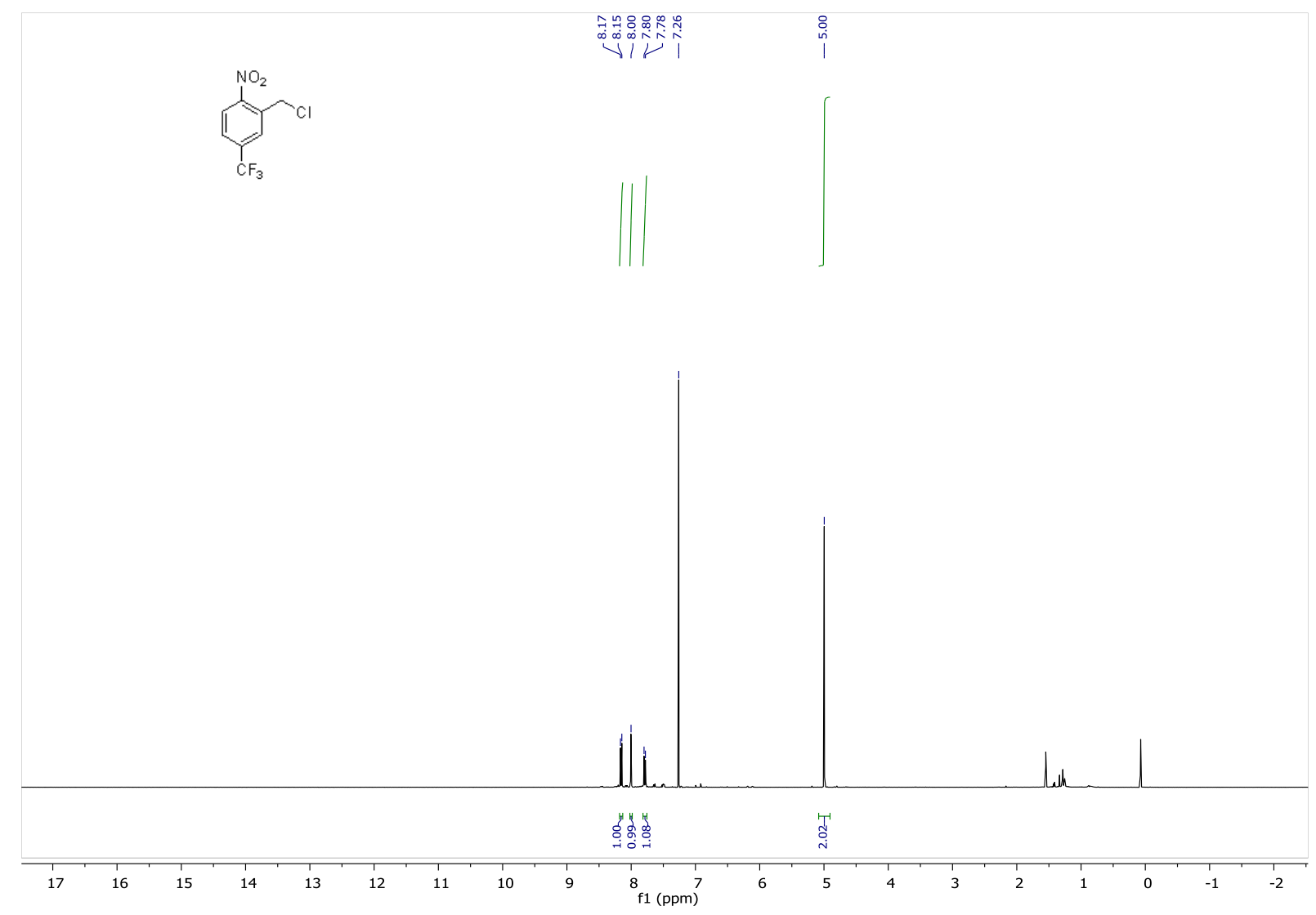

2d, ${ }^{13} \mathrm{C} \mathrm{NMR}\left(\mathrm{CDCl}_{3}, 100 \mathrm{MHz}\right)$

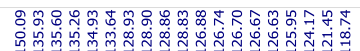

政

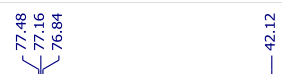

$\underbrace{\mathrm{NO}}_{\mathrm{C}_{3}} \mathrm{Cl}$
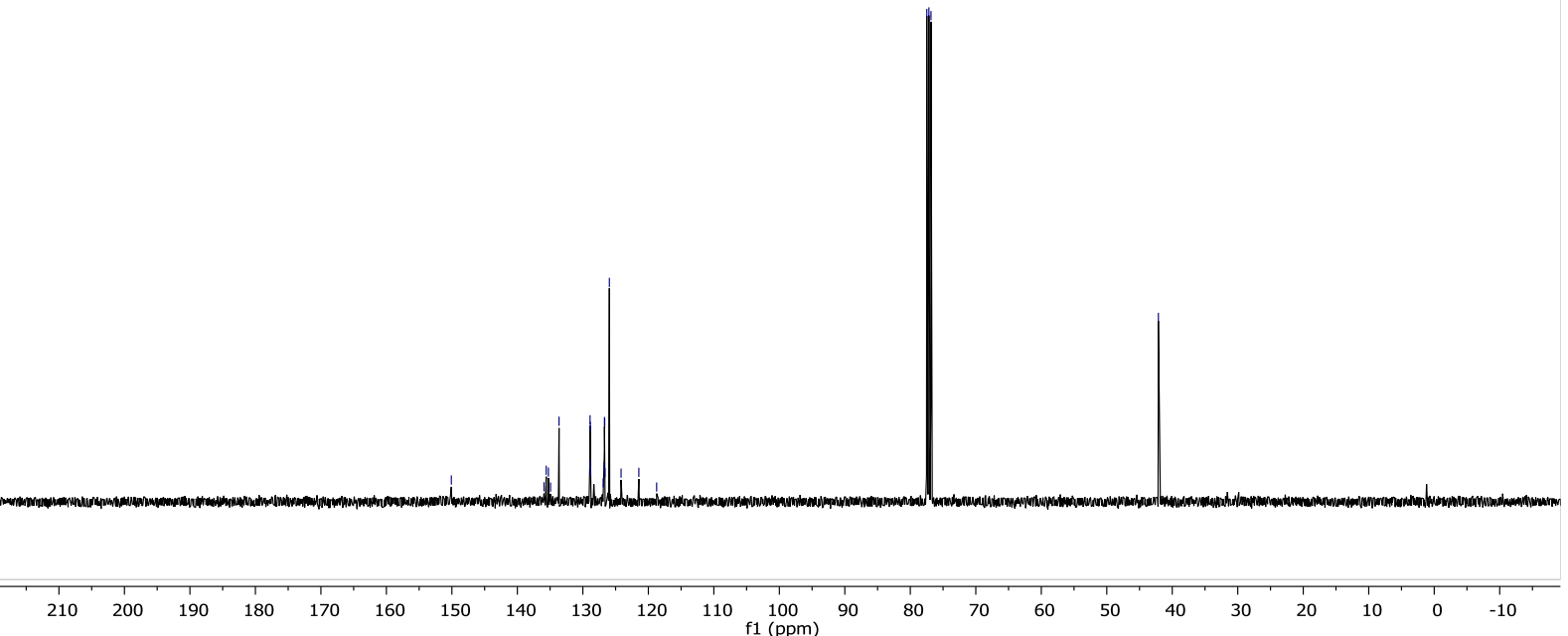

2d, ${ }^{19} \mathrm{~F} \mathrm{NMR}\left(\mathrm{CDCl}_{3}, 376 \mathrm{MHz}\right)$ 


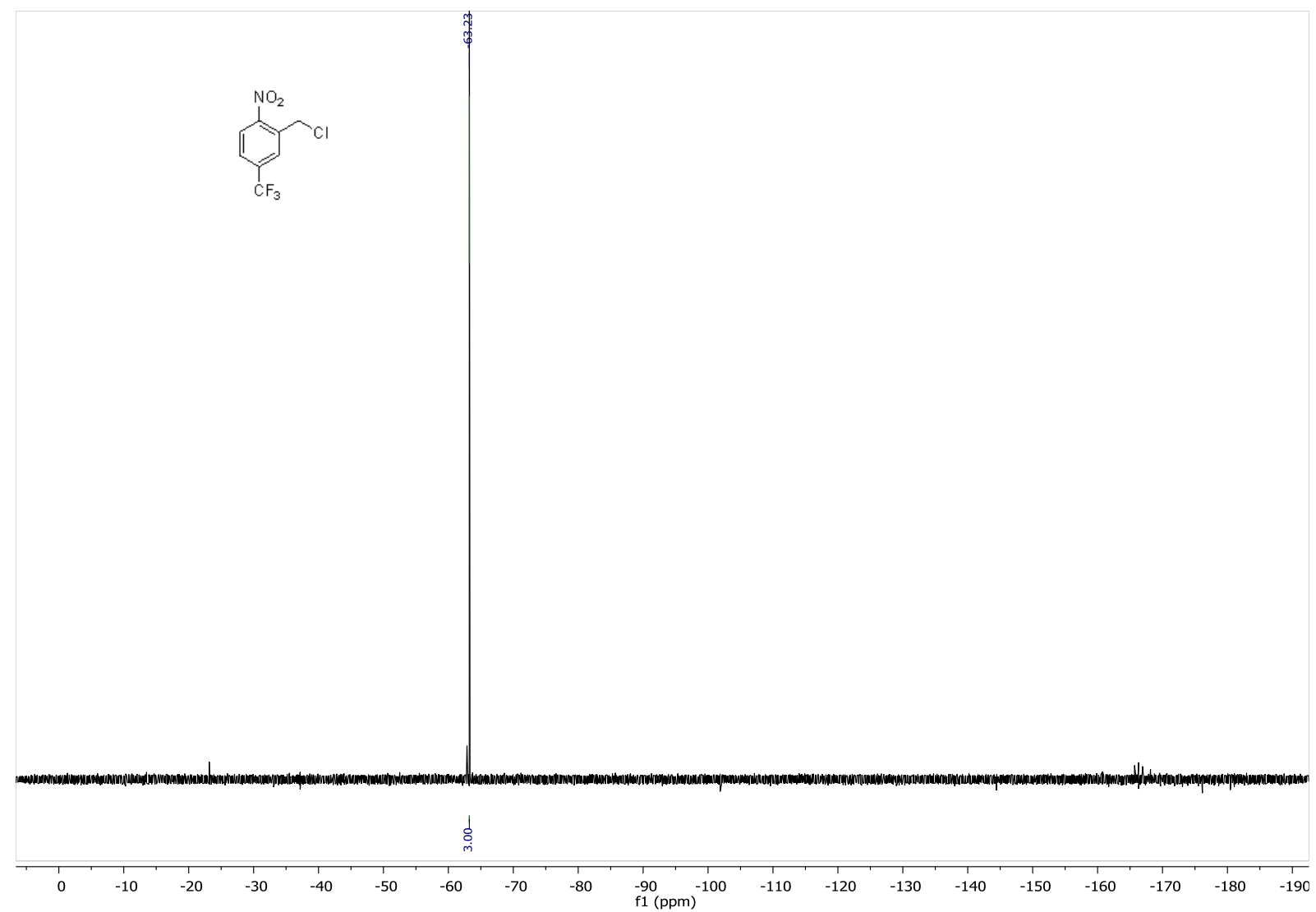

2e, ${ }^{19} \mathrm{~F} \mathrm{NMR}\left(\mathrm{CDCl}_{3}, 376 \mathrm{MHz}\right)$

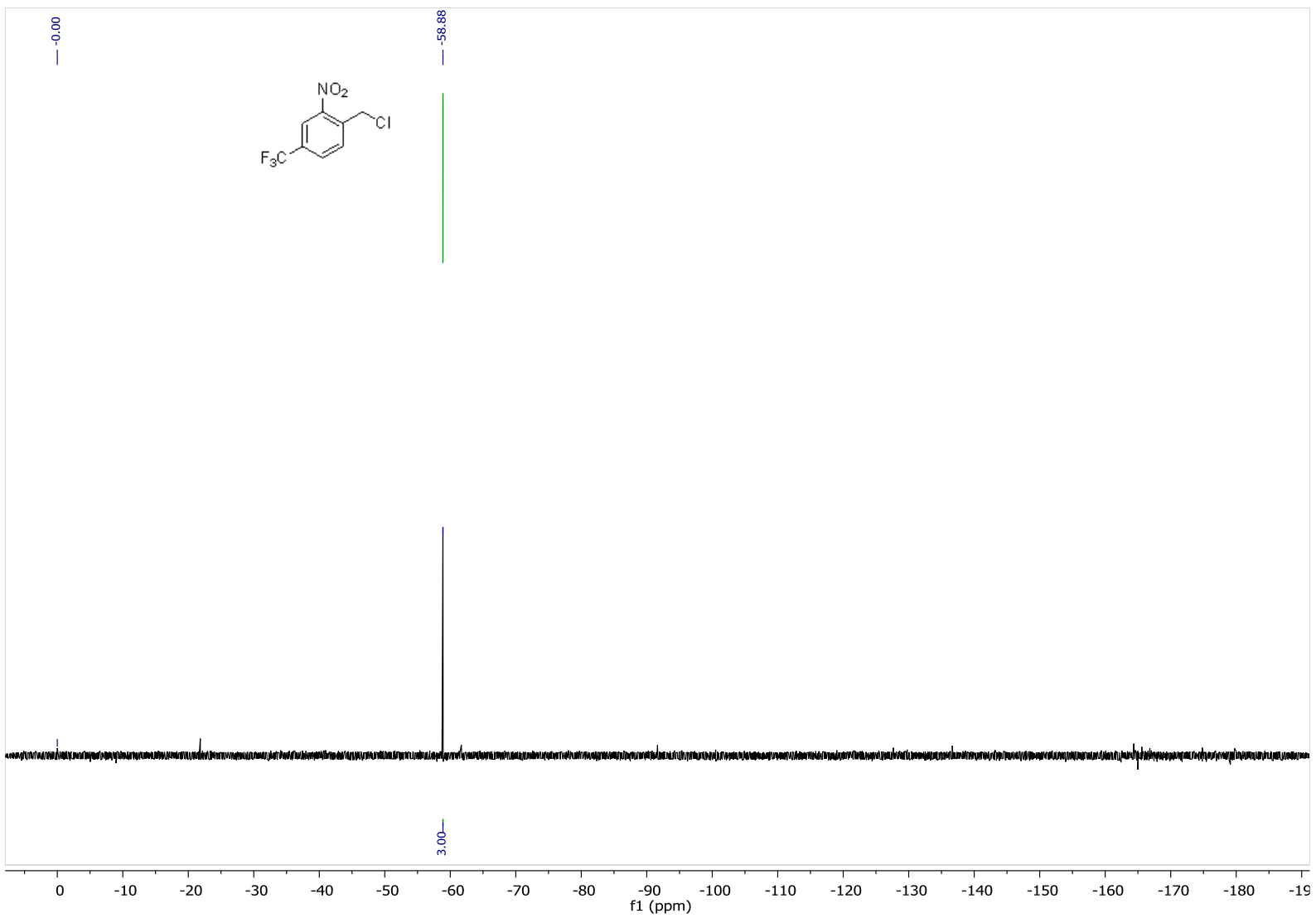

2f, ${ }^{1} \mathrm{H} \mathrm{NMR}\left(\mathrm{CDCl}_{3}, 400 \mathrm{MHz}\right)$ 


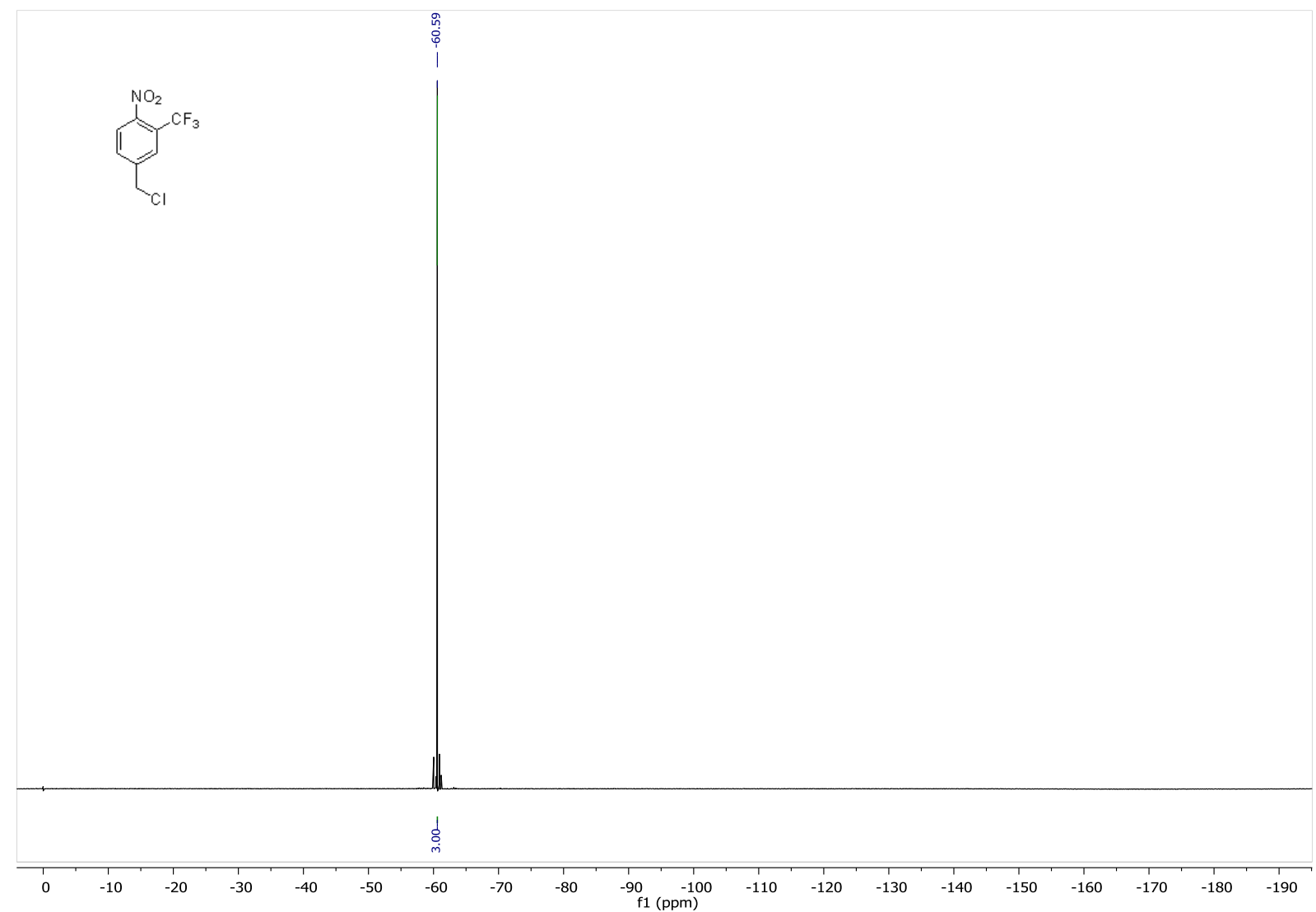

2h, ${ }^{1} \mathrm{H} \mathrm{NMR}\left(\mathrm{CDCl}_{3}, 400 \mathrm{MHz}\right)$

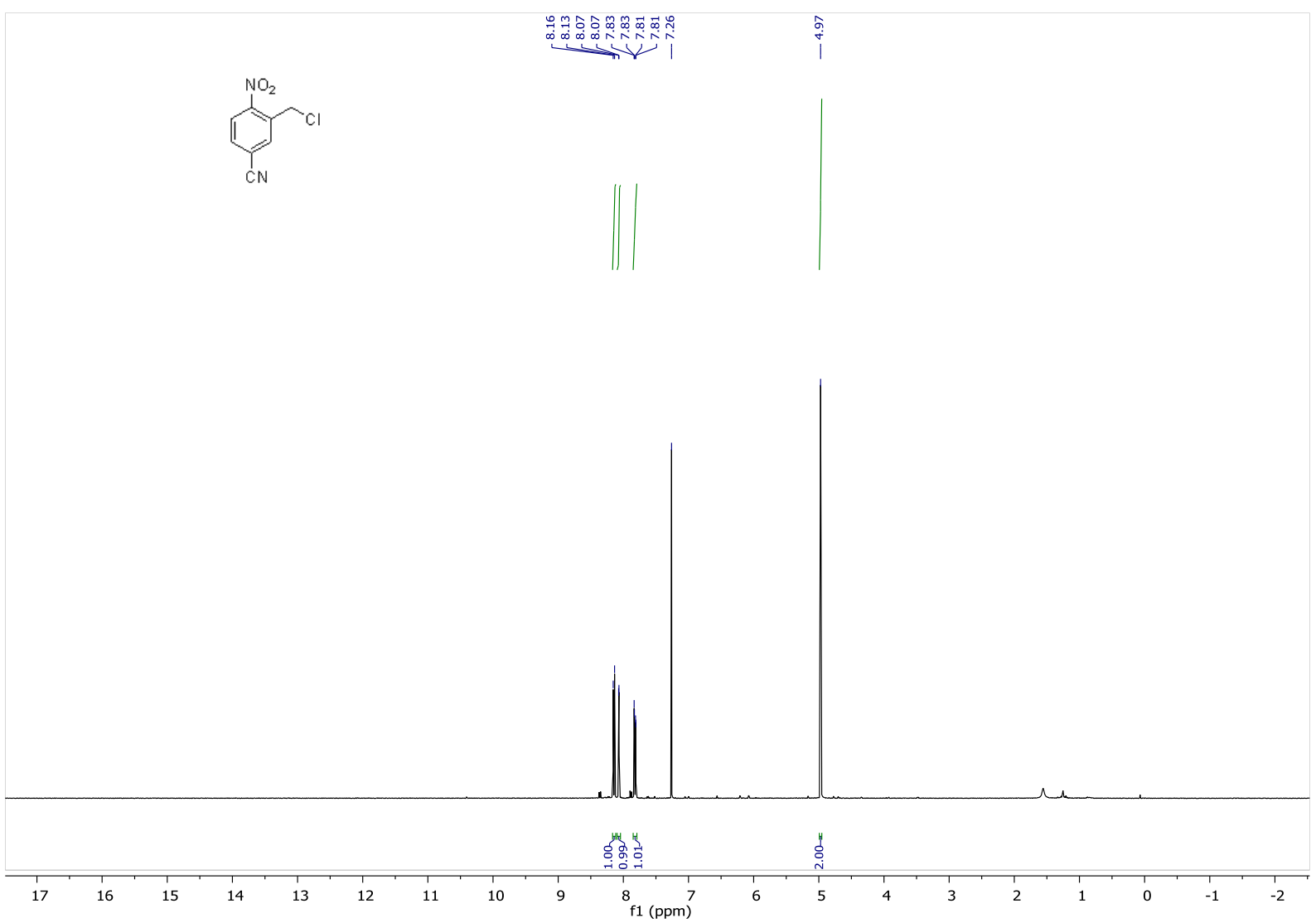

2h, ${ }^{13} \mathrm{C} \mathrm{NMR}\left(\mathrm{CDCl}_{3}, 100 \mathrm{MHz}\right)$ 

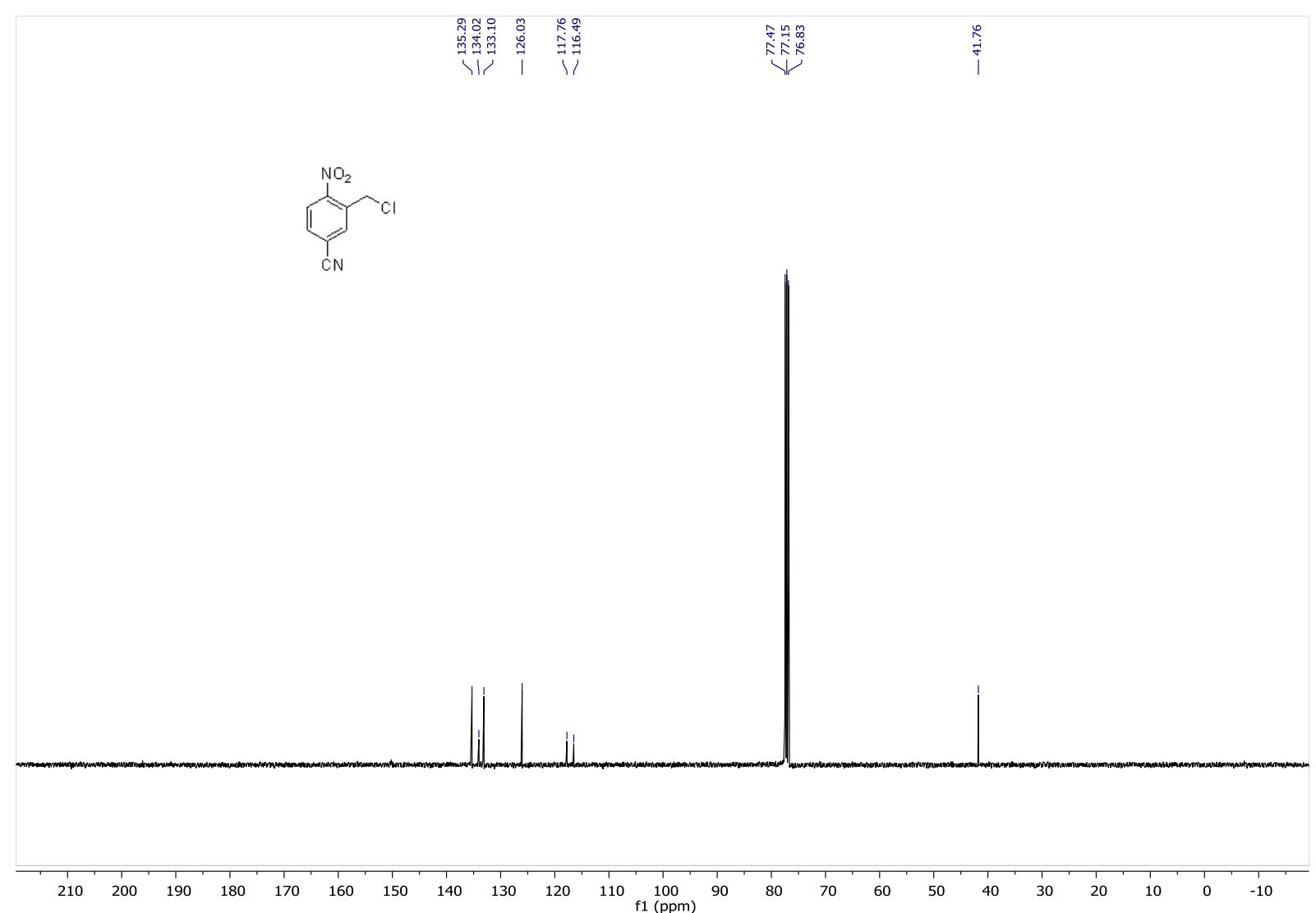

2h, ${ }^{1} \mathrm{H} \mathrm{NMR}\left(\mathrm{CDCl}_{3}, 400 \mathrm{MHz}\right)$

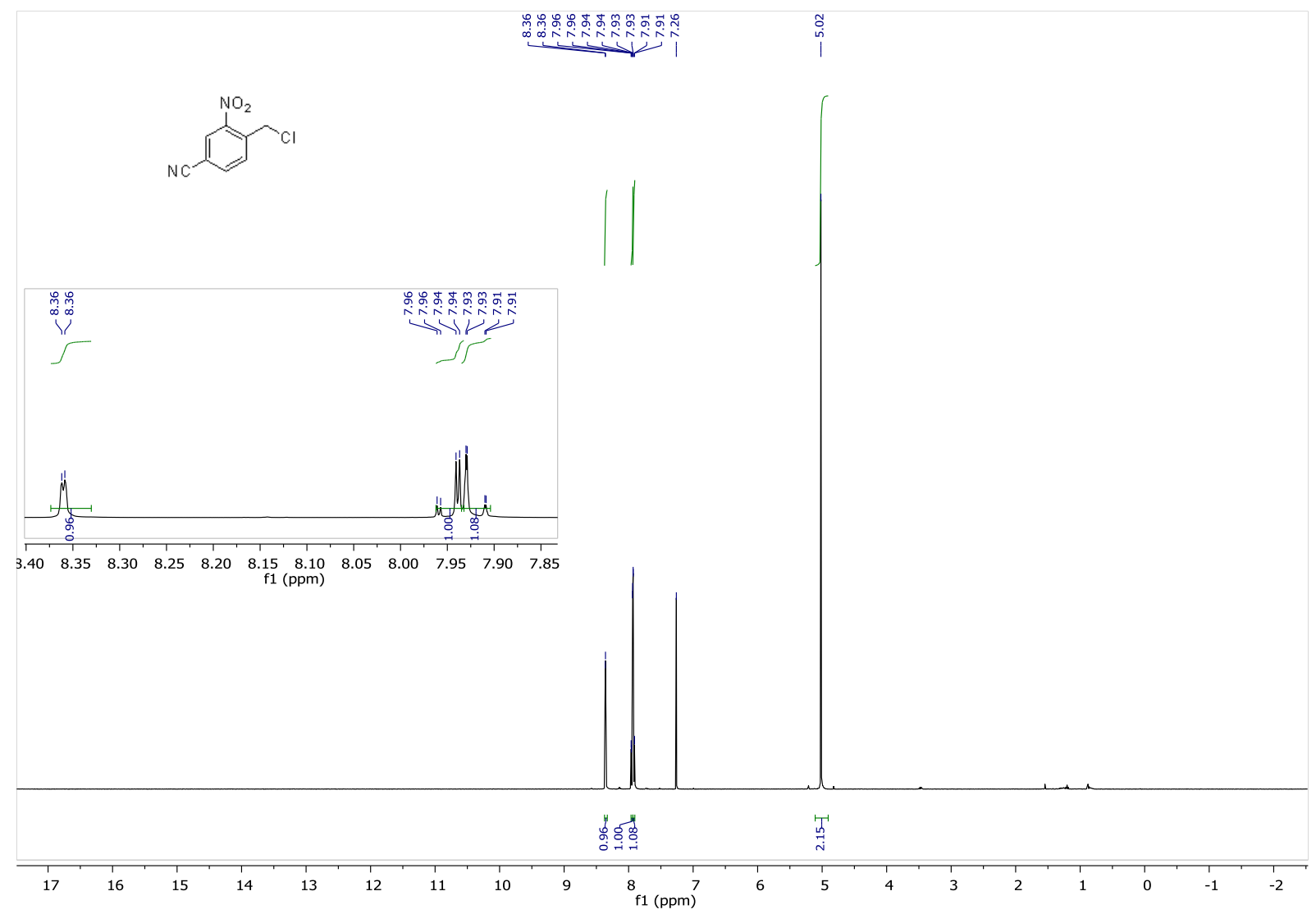

2i, ${ }^{13} \mathrm{C} \mathrm{NMR}\left(\mathrm{CDCl}_{3}, 100 \mathrm{MHz}\right)$ 

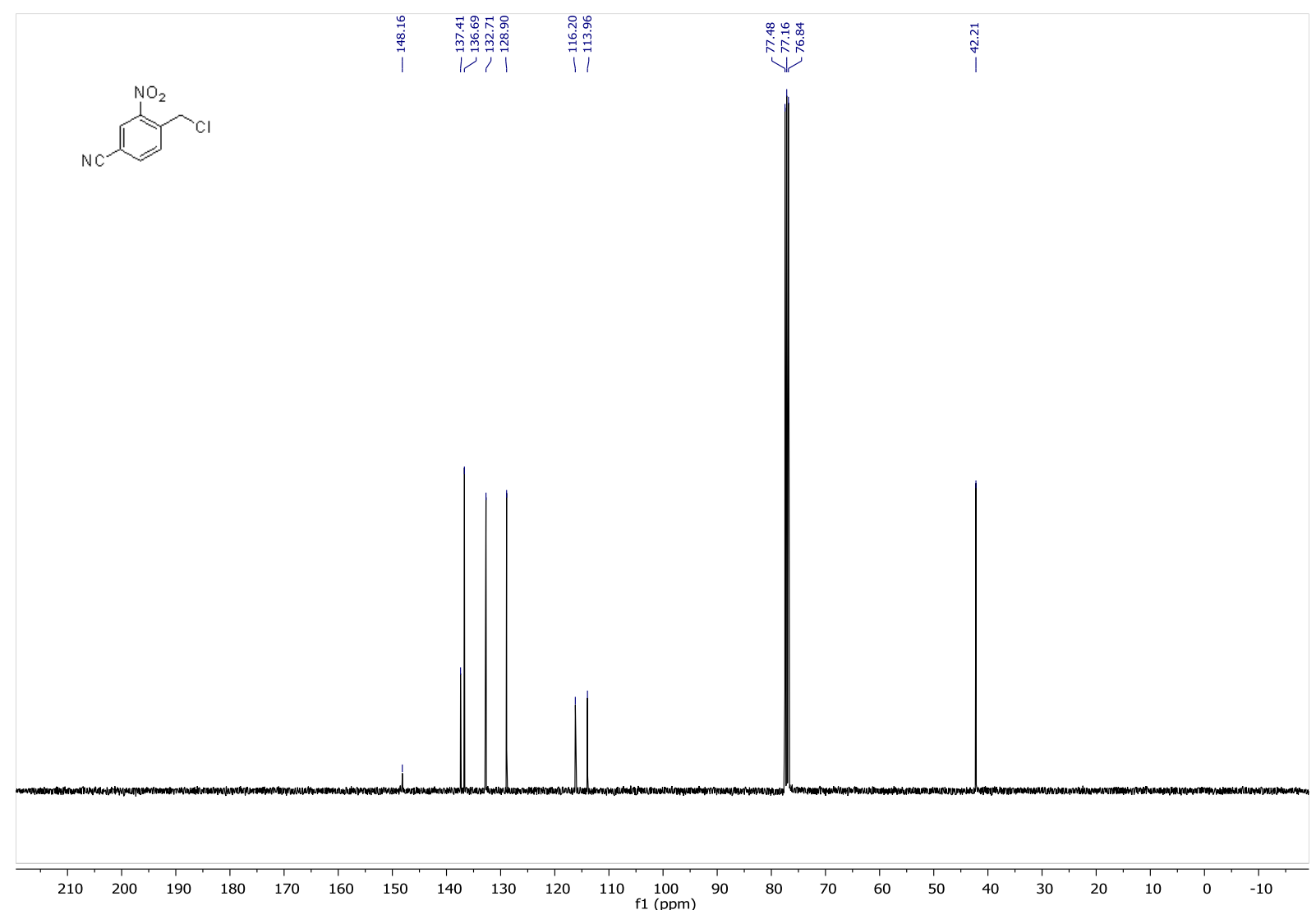

2k, ${ }^{1} \mathrm{H} \mathrm{NMR}\left(\mathrm{CDCl}_{3}, 400 \mathrm{MHz}\right)$

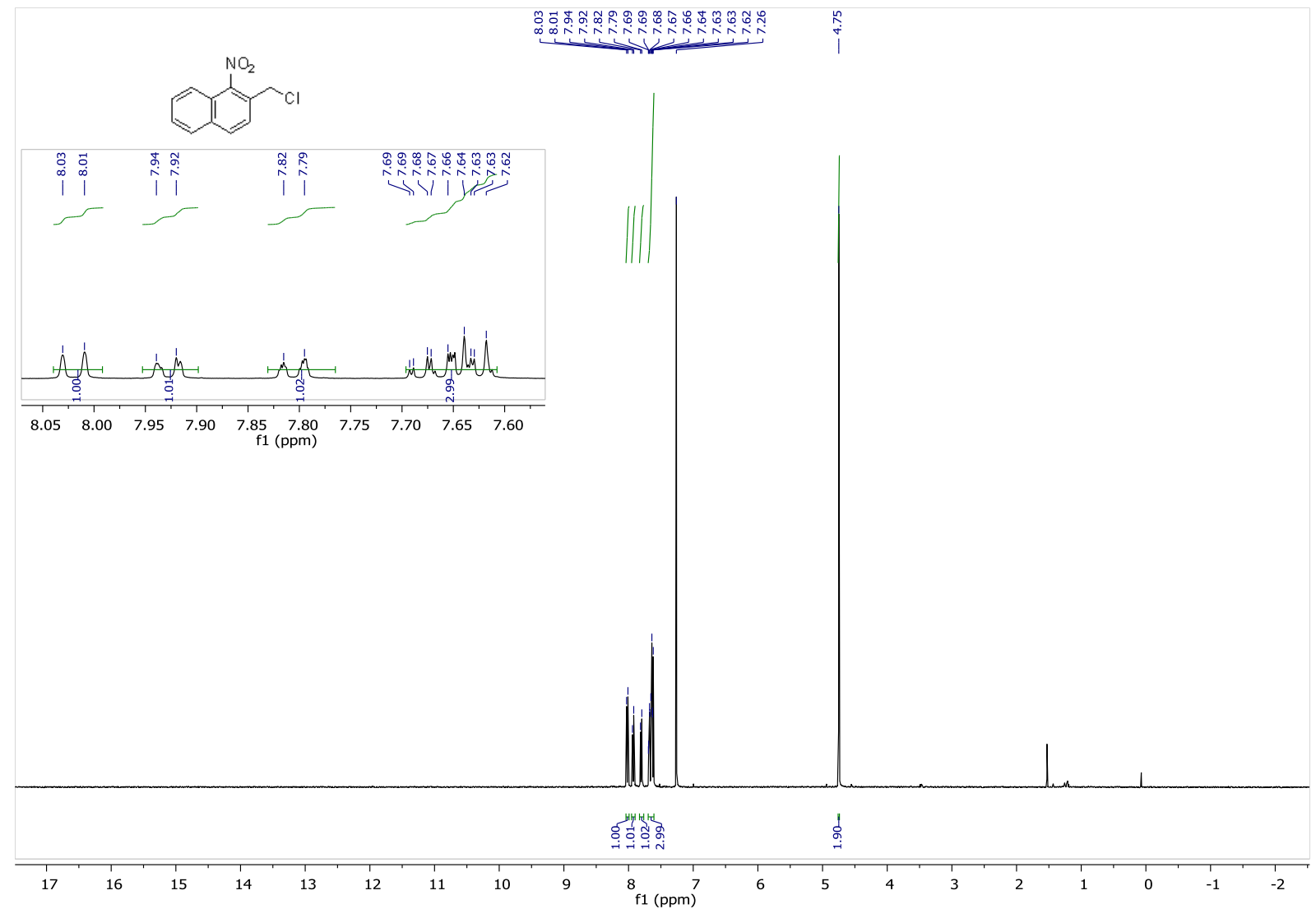

2k, ${ }^{13} \mathrm{C} \mathrm{NMR}\left(\mathrm{CDCl}_{3}, 100 \mathrm{MHz}\right)$ 


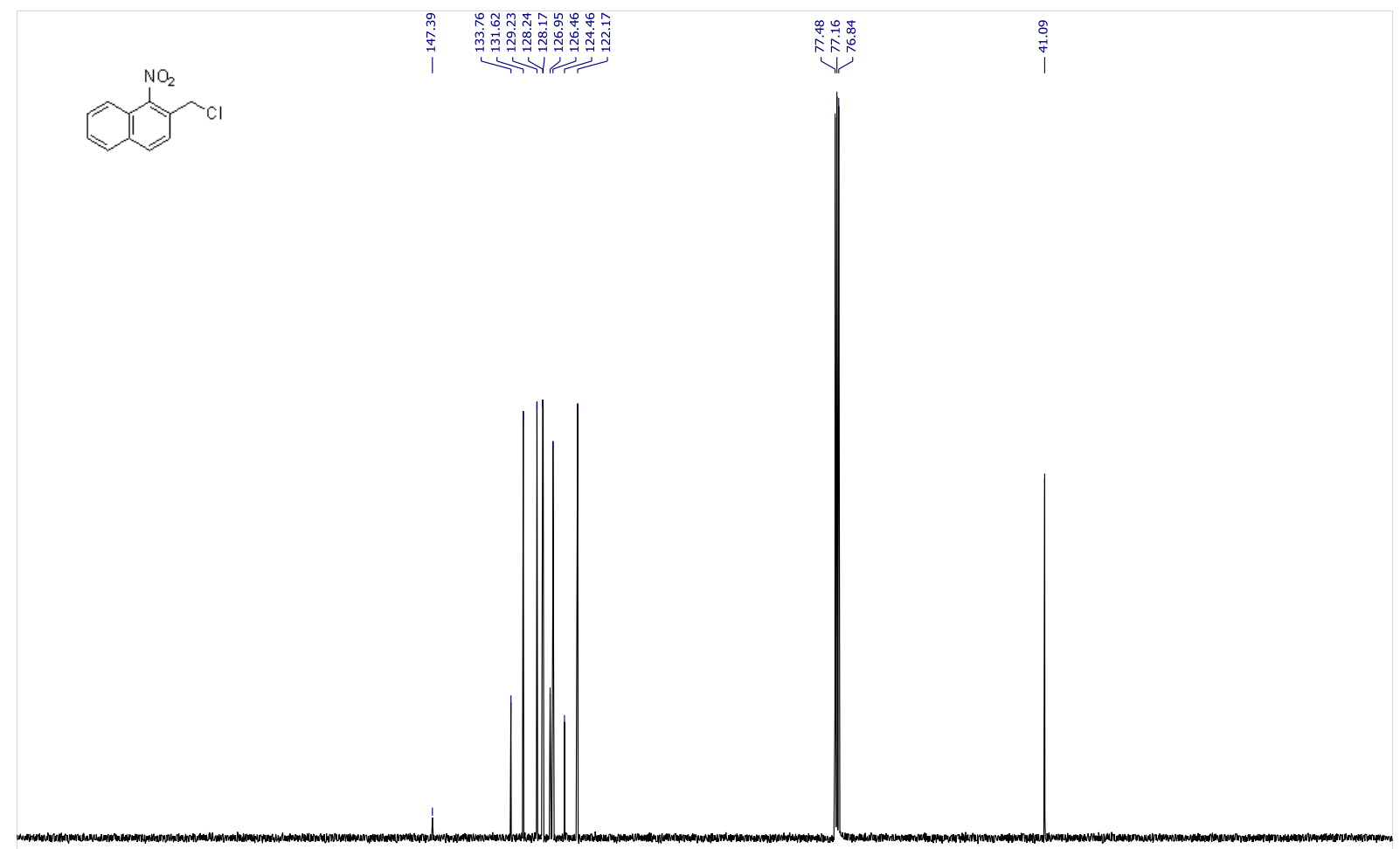

$\begin{array}{lllllllllllllllllllllllllllllllllll}210 & 200 & 190 & 180 & 170 & 160 & 150 & 140 & 130 & 120 & 110 & 100 & 90 & 80 & 70 & 60 & 50 & 40 & 30 & 20 & 10 & 0 & -10 & \end{array}$

2-(Dichloromethyl)naphthalene: ${ }^{1} \mathrm{H} \mathrm{NMR}\left(\mathrm{CDCl}_{3}, 400 \mathrm{MHz}\right)$

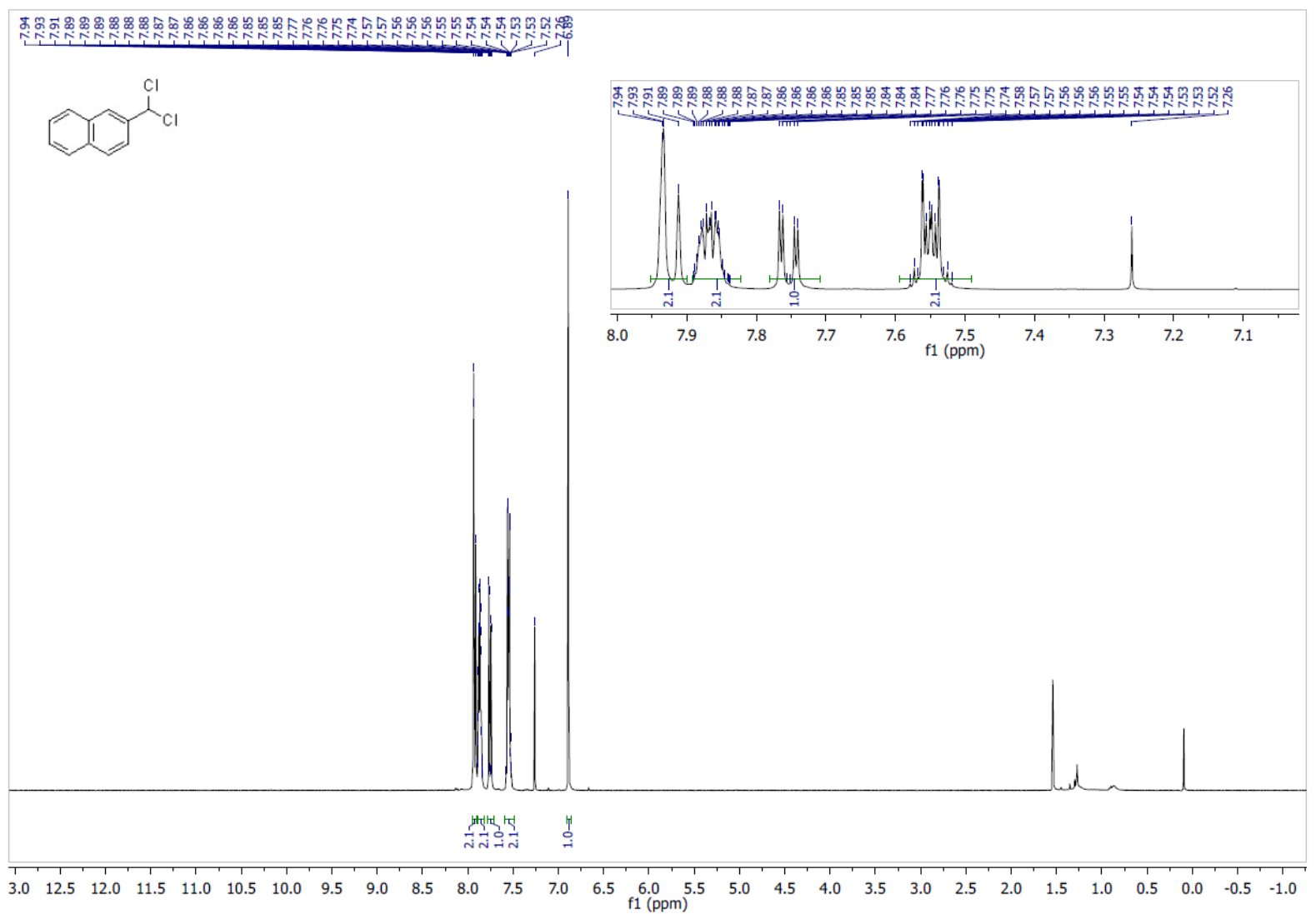

2-(Dichloromethyl)naphthalene: ${ }^{13} \mathrm{C} \mathrm{NMR}\left(\mathrm{CDCl}_{3}, 100.6 \mathrm{MHz}\right)$ 


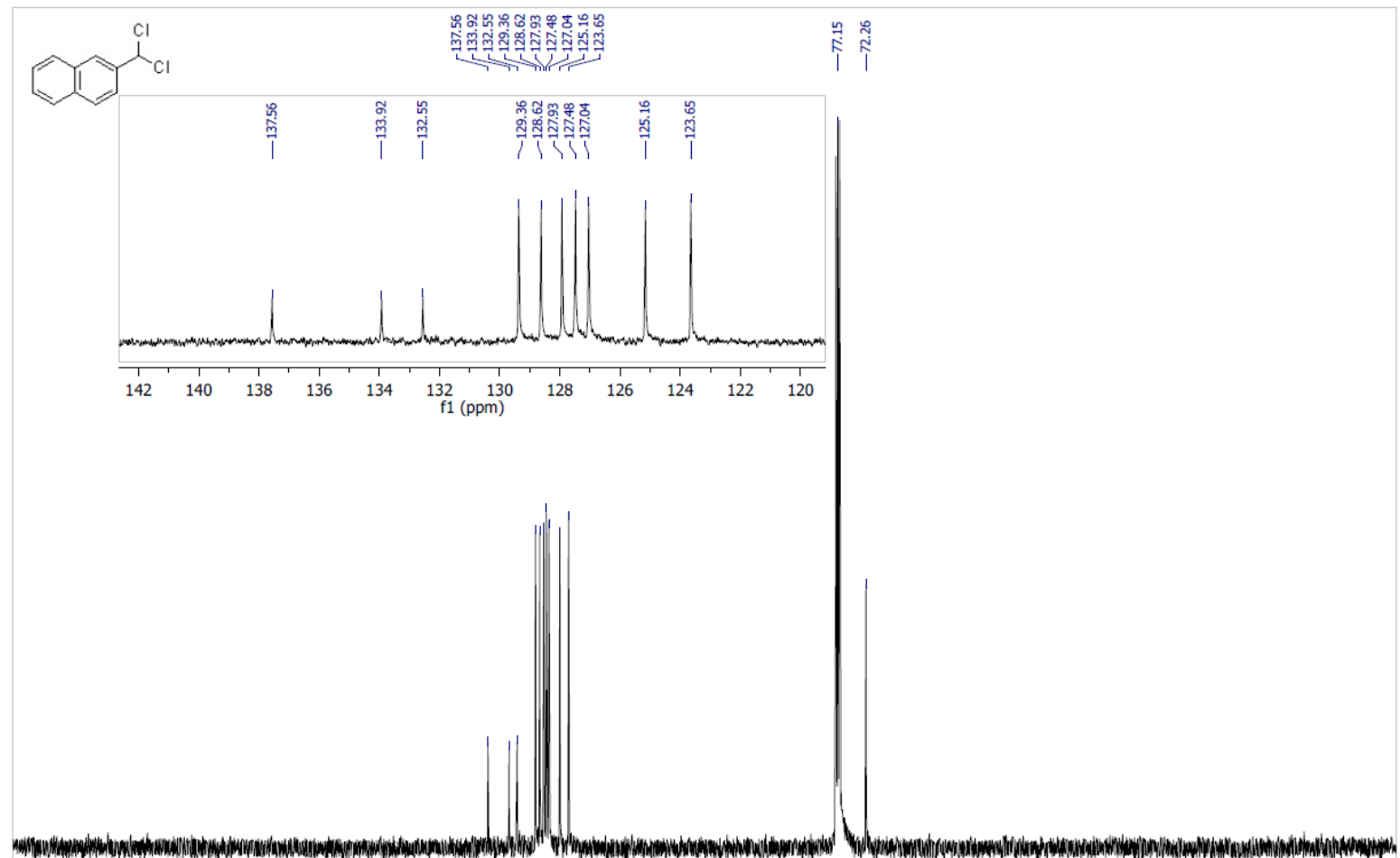

$\begin{array}{lllllllllllllllllllllllll}210 & 200 & 190 & 180 & 170 & 160 & 150 & 140 & 130 & 120 & 110 & \underset{\mathrm{f} 1(\mathrm{ppm})}{100} & 90 & 80 & 70 & 60 & 50 & 40 & 30 & 20 & 10 & 0 & -10\end{array}$

2l, ${ }^{1} \mathrm{H} \mathrm{NMR}\left(\mathrm{CDCl}_{3}, 400 \mathrm{MHz}\right)$

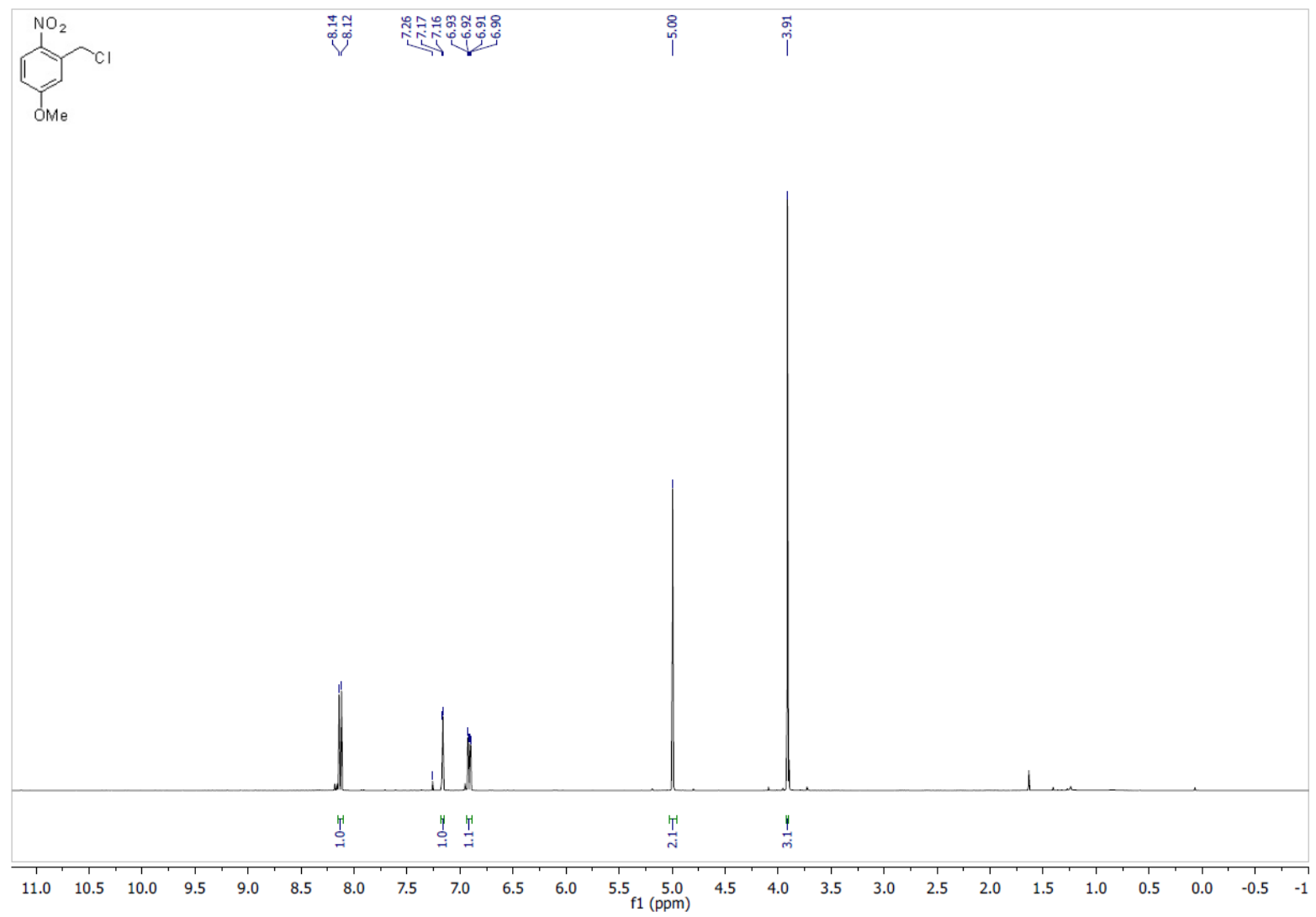

2l, ${ }^{13} \mathrm{C} \mathrm{NMR}\left(\mathrm{CDCl}_{3}, 100 \mathrm{MHz}\right)$ 


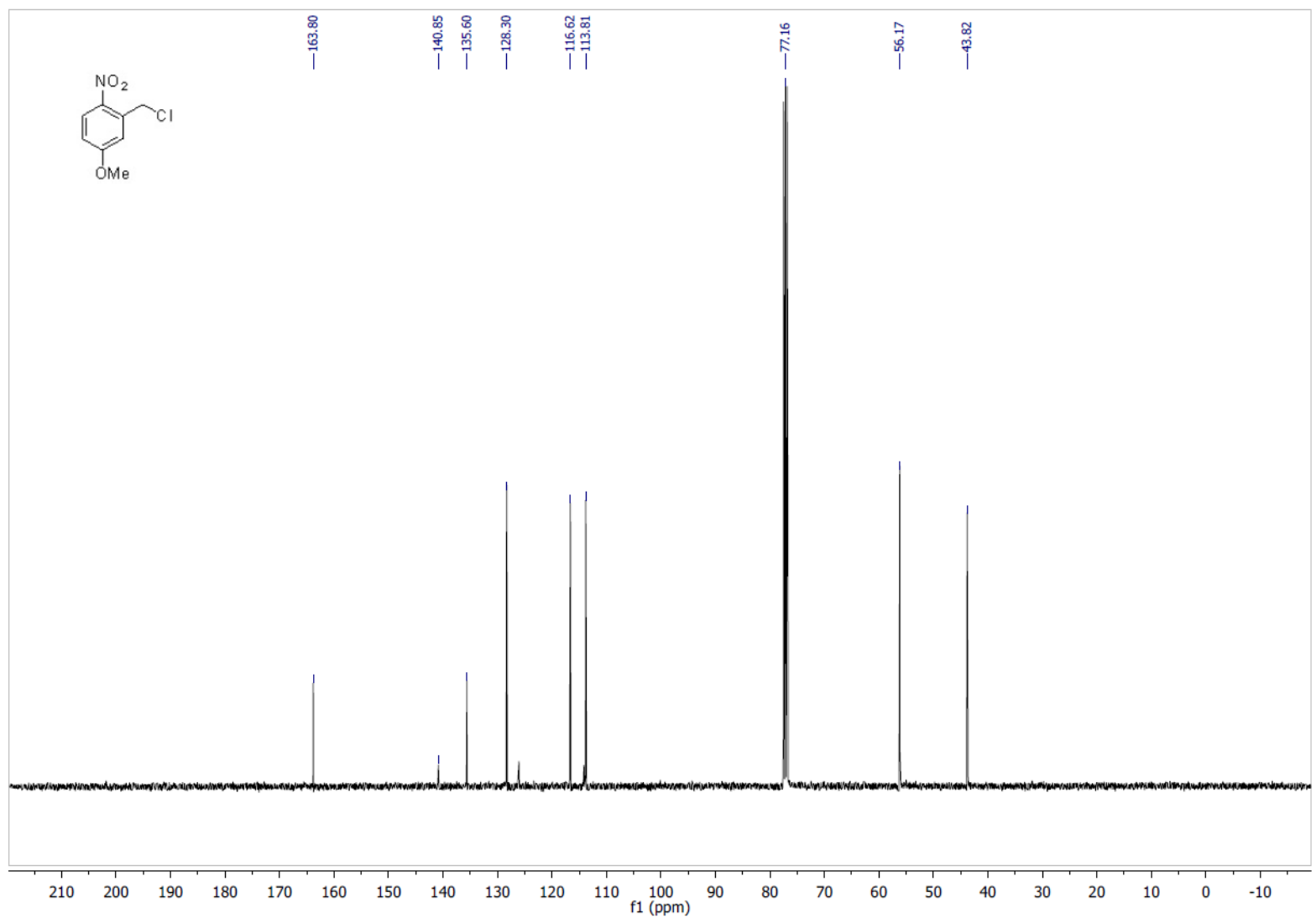

2m, ${ }^{1} \mathrm{H} \mathrm{NMR}\left(\mathrm{CDCl}_{3}, 400 \mathrm{MHz}\right)$

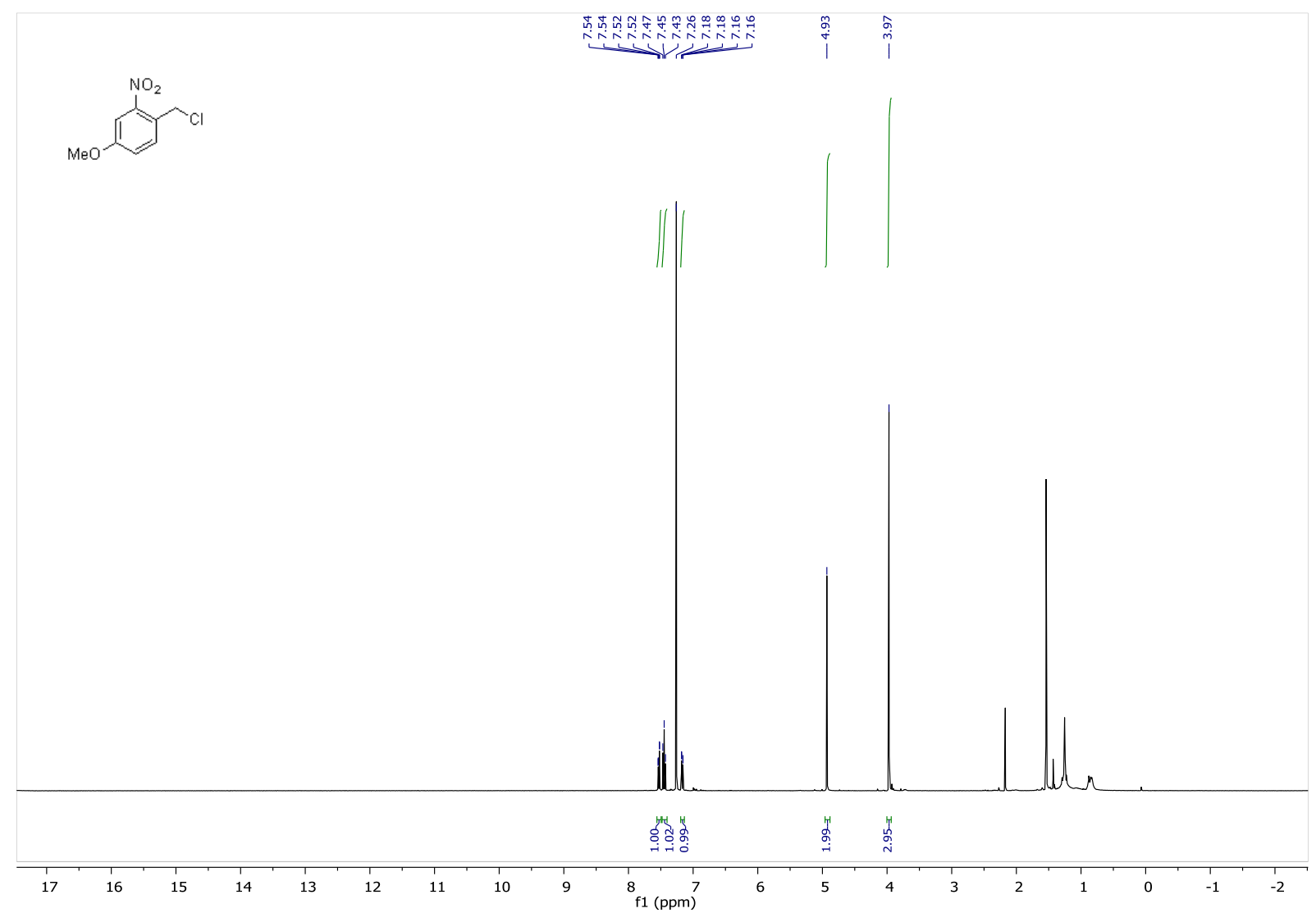

2m, ${ }^{13} \mathrm{C}$ NMR (APT, $\mathrm{CDCl}_{3}, 100 \mathrm{MHz}$ ) 


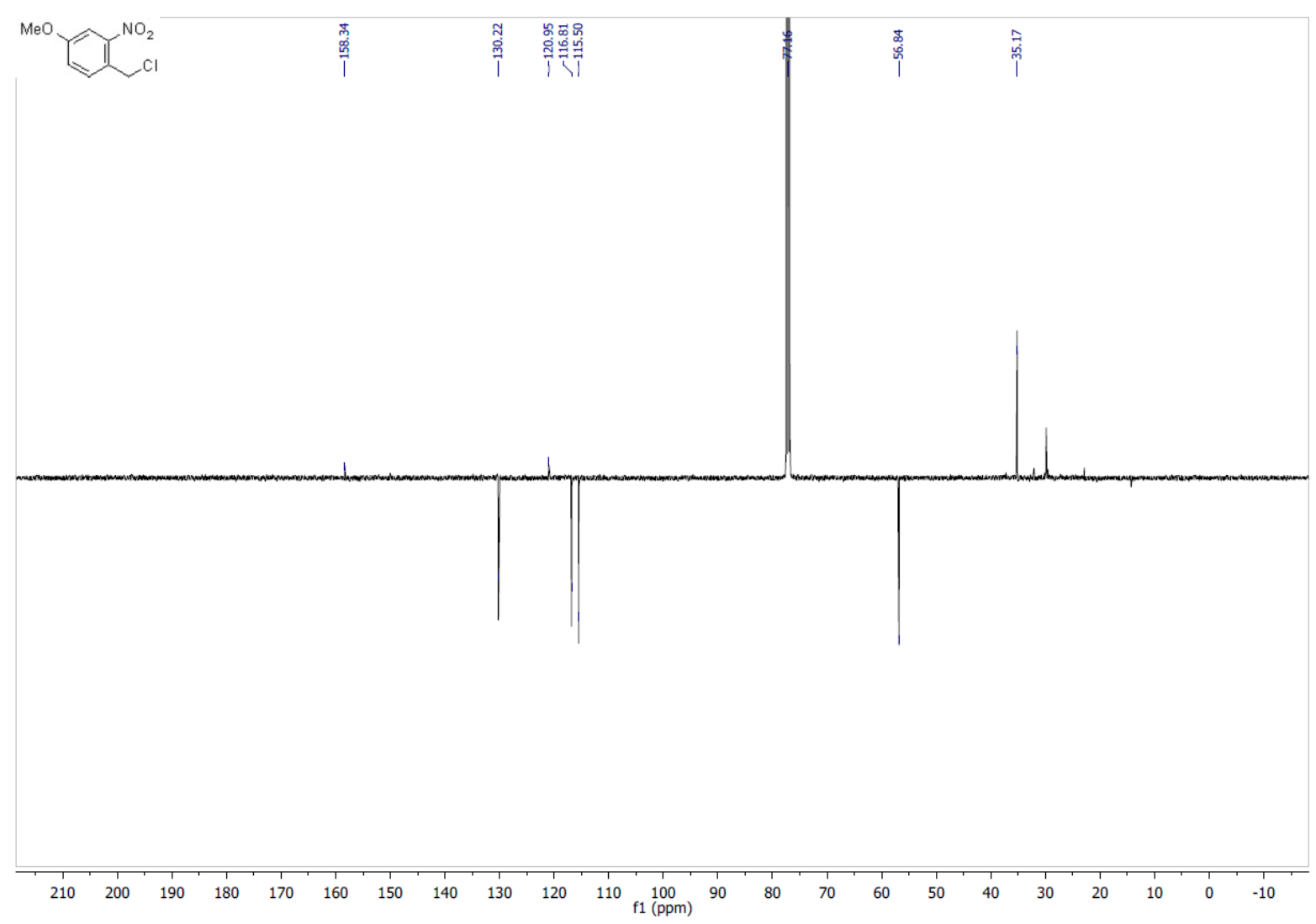

2n, ${ }^{1} \mathrm{H} \mathrm{NMR}\left(\mathrm{CDCl}_{3}, 400 \mathrm{MHz}\right)$

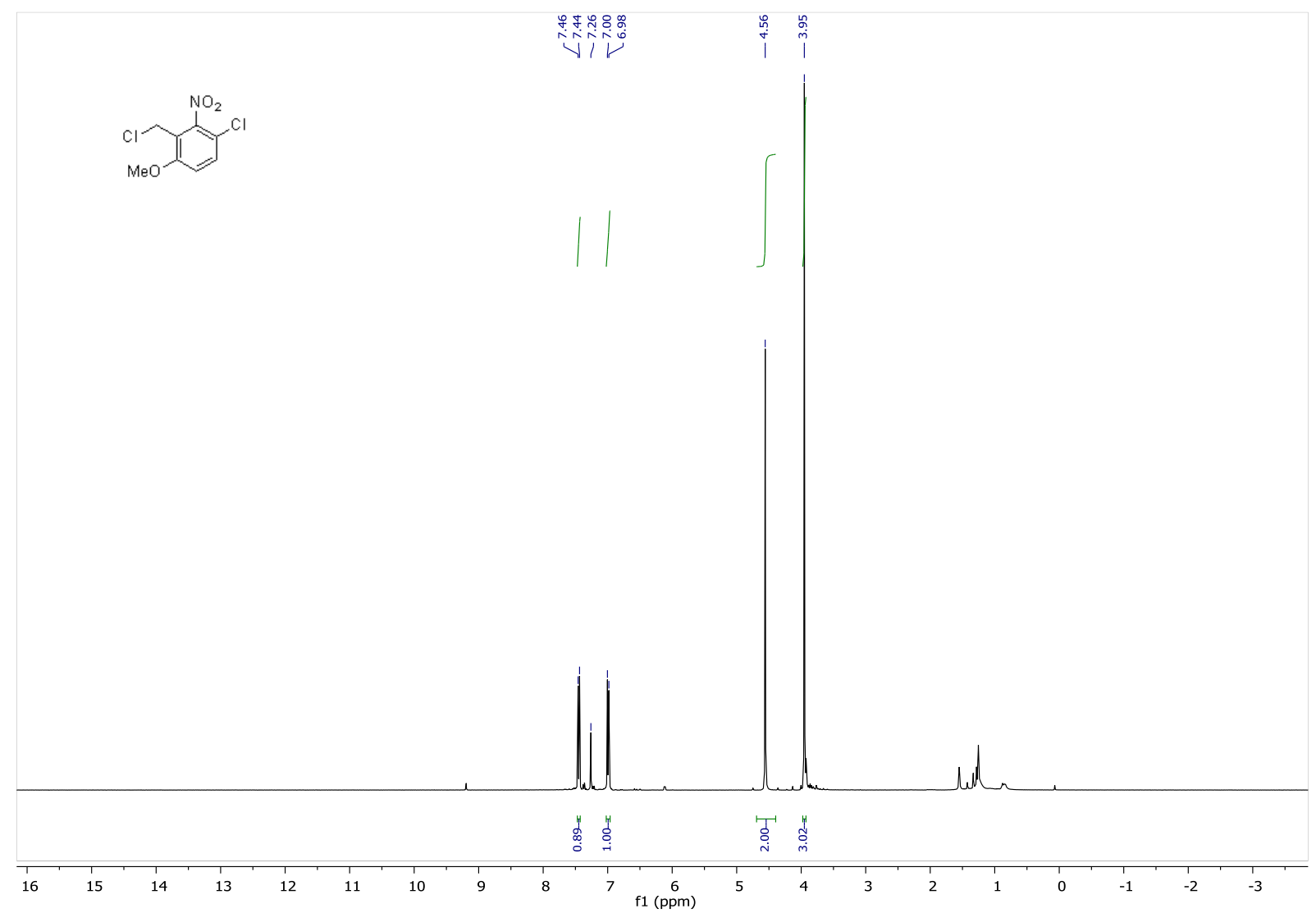

2n, ${ }^{13} \mathrm{C} \mathrm{NMR}\left(\mathrm{CDCl}_{3}, 100 \mathrm{MHz}\right)$ 


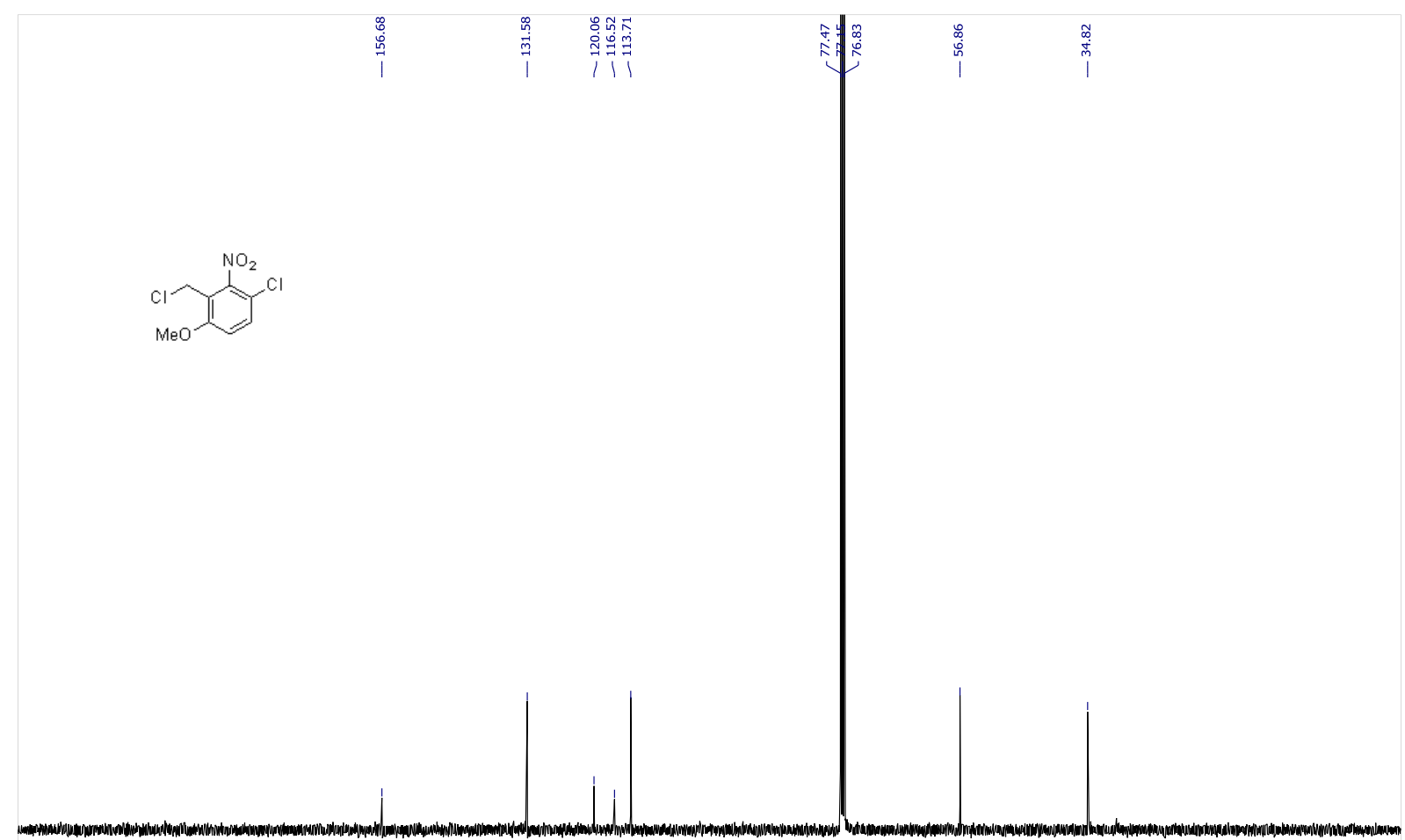

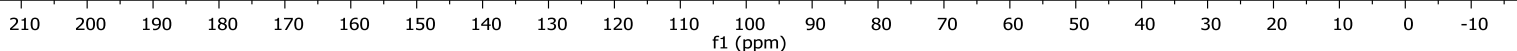

2o, ${ }^{1} \mathrm{H} \mathrm{NMR}\left(\mathrm{CDCl}_{3}, 400 \mathrm{MHz}\right)$

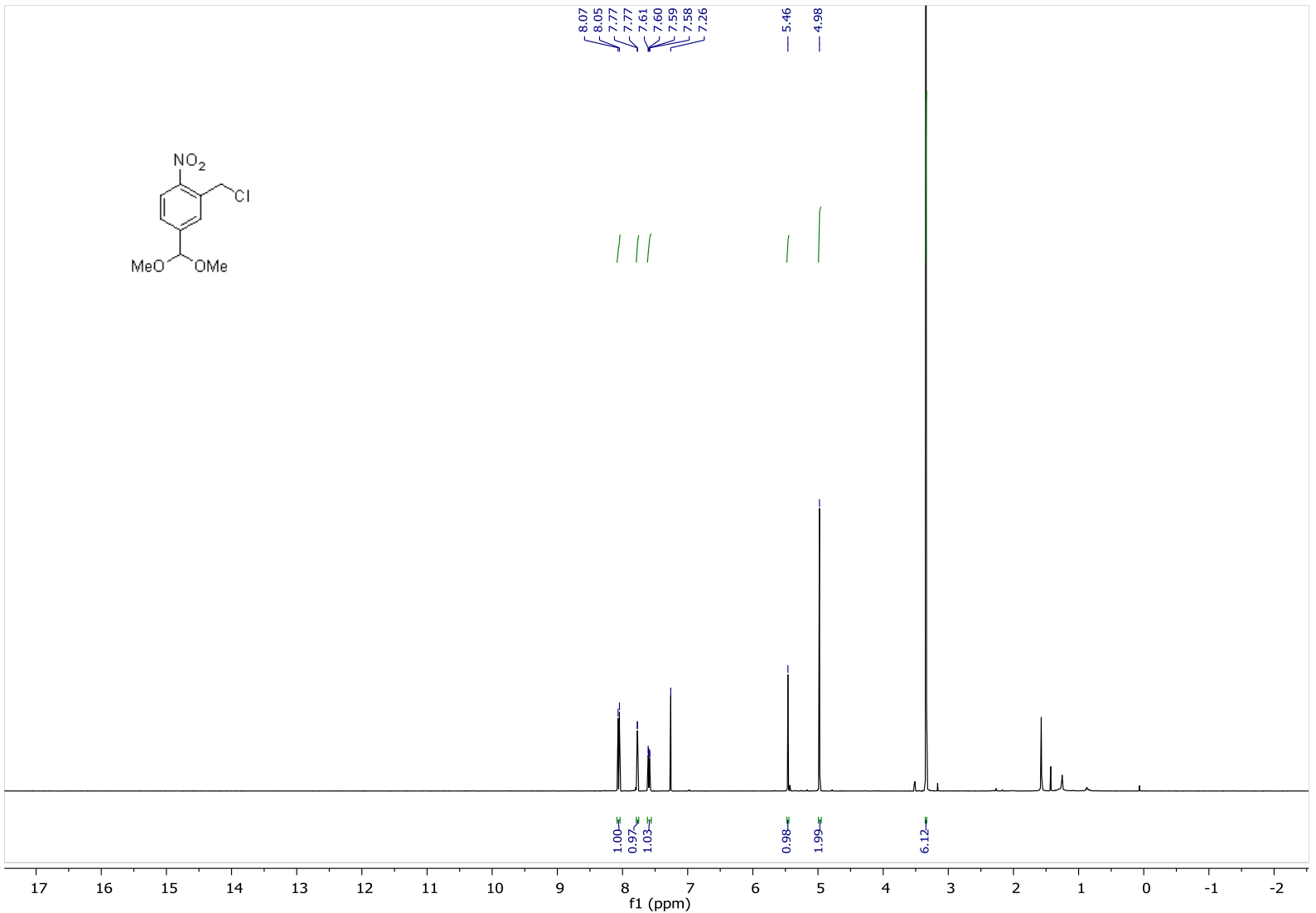

2o, ${ }^{13} \mathrm{C} \mathrm{NMR}\left(\mathrm{CDCl}_{3}, 100 \mathrm{MHz}\right)$ 


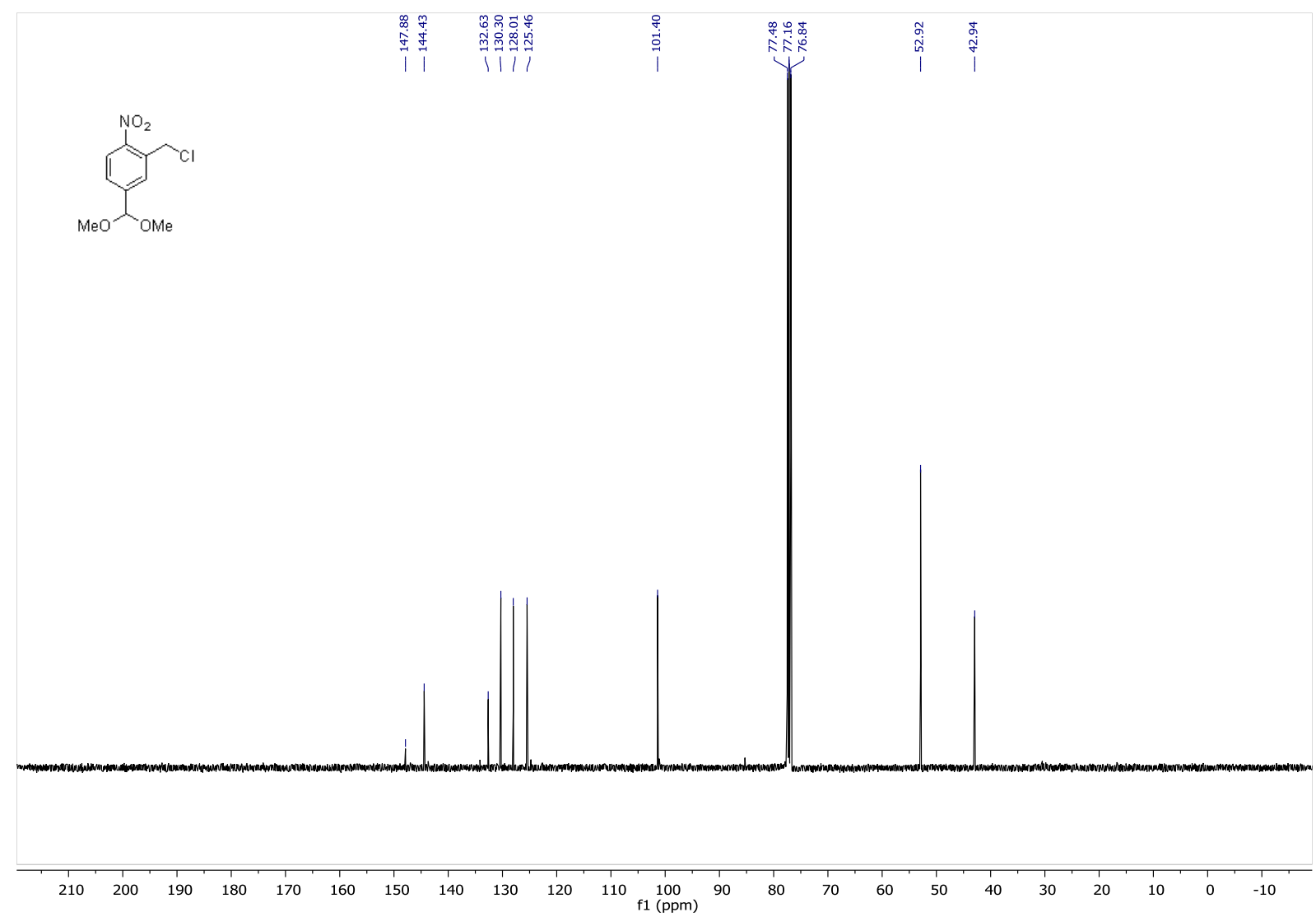

2p, ${ }^{1} \mathrm{H} \mathrm{NMR}\left(\mathrm{CDCl}_{3}, 400 \mathrm{MHz}\right)$

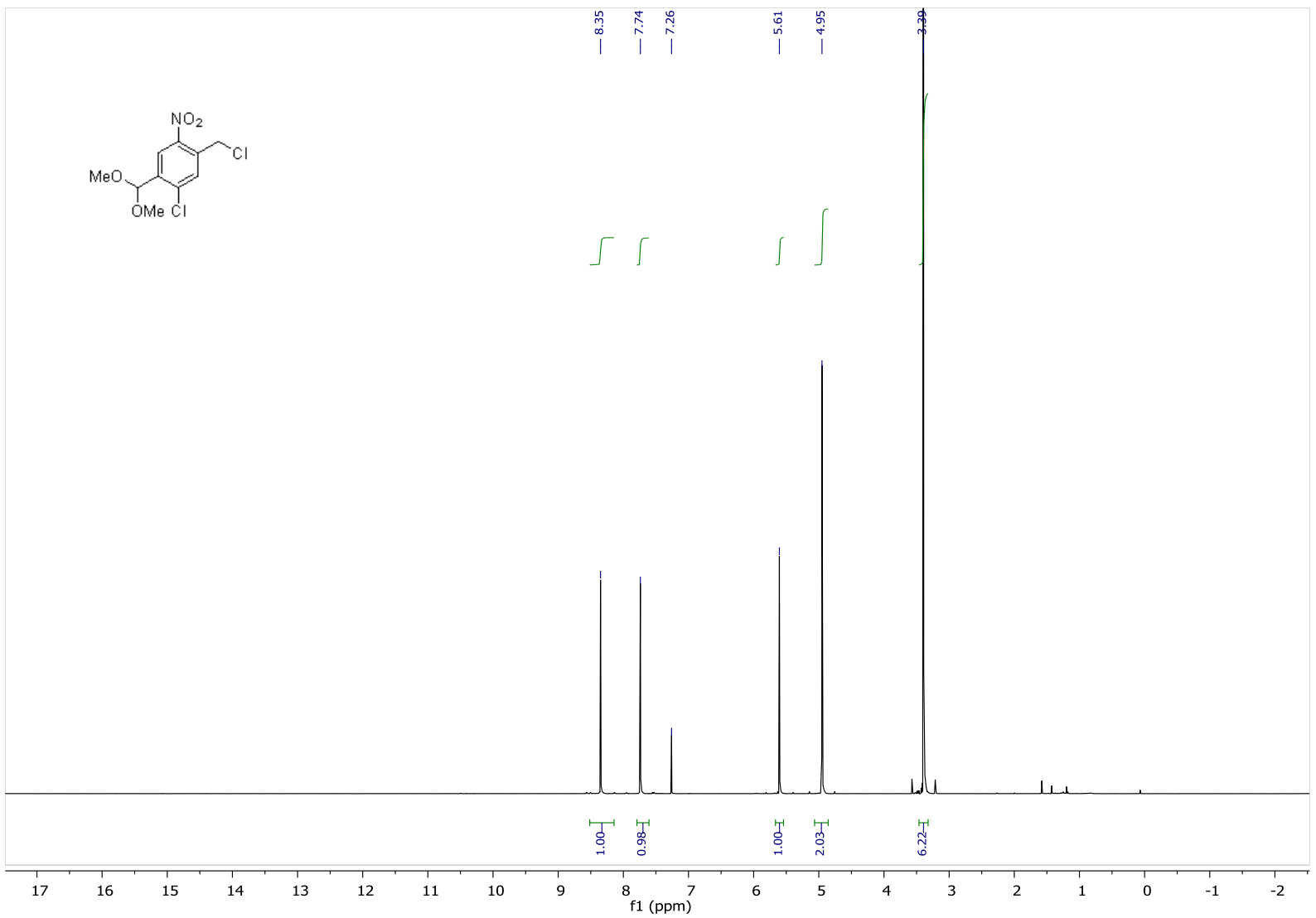

2p, ${ }^{13} \mathrm{C} \mathrm{NMR}\left(\mathrm{CDCl}_{3}, 100 \mathrm{MHz}\right)$ 

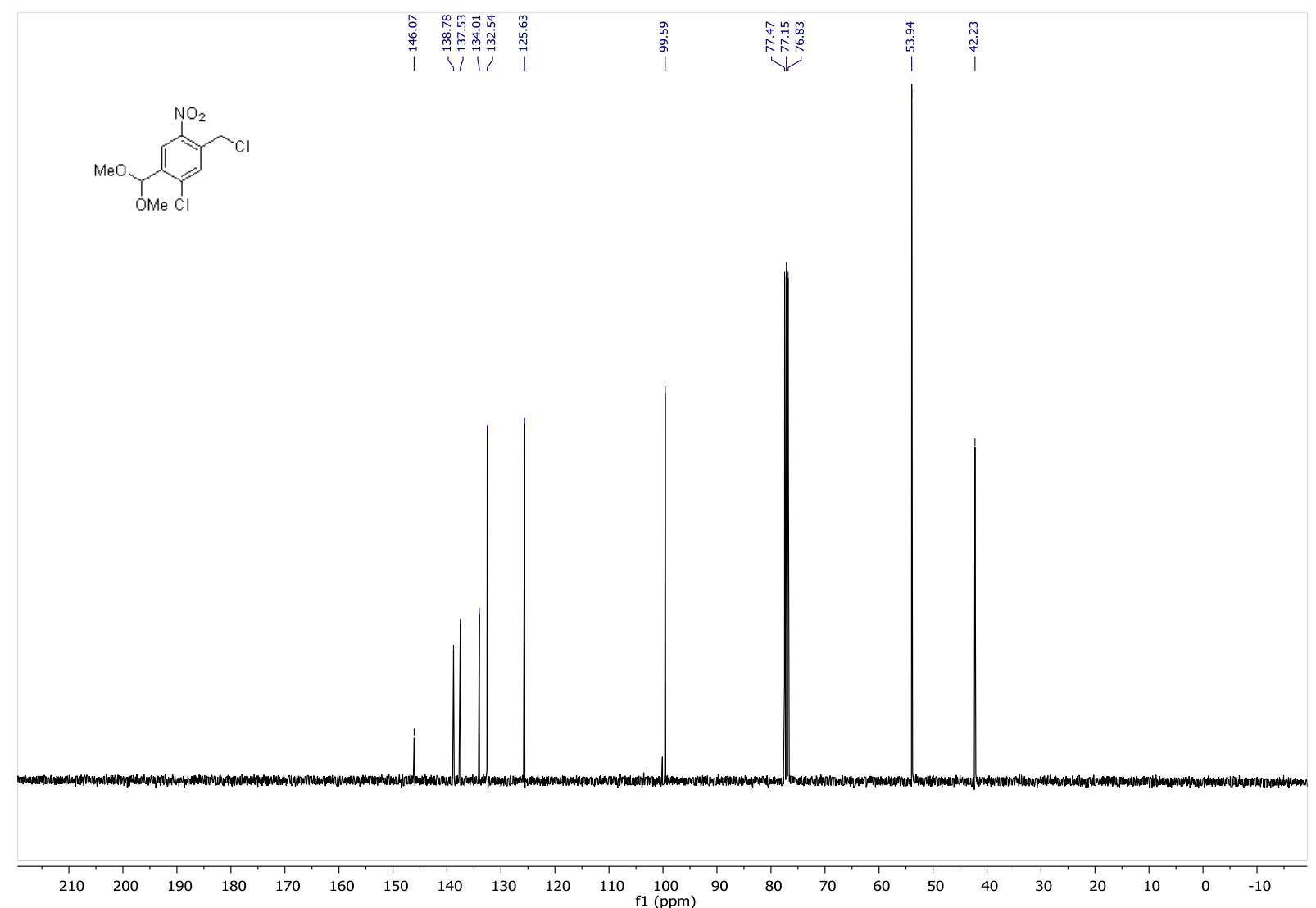

2q, ${ }^{1} \mathrm{H} \mathrm{NMR}\left(\mathrm{CDCl}_{3}, 400 \mathrm{MHz}\right)$

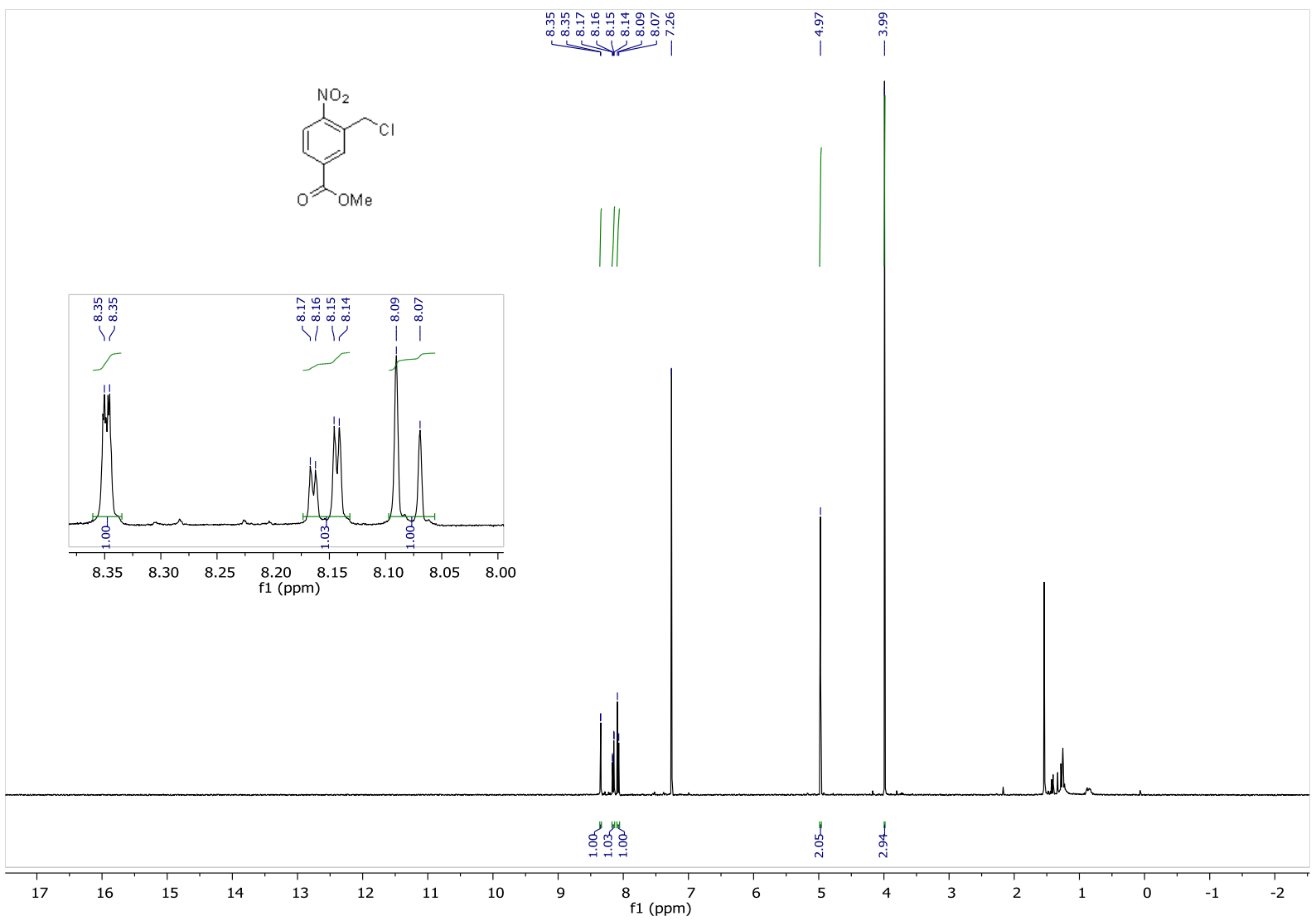

2q, ${ }^{13} \mathrm{C} \mathrm{NMR}\left(\mathrm{CDCl}_{3}, 100 \mathrm{MHz}\right)$ 


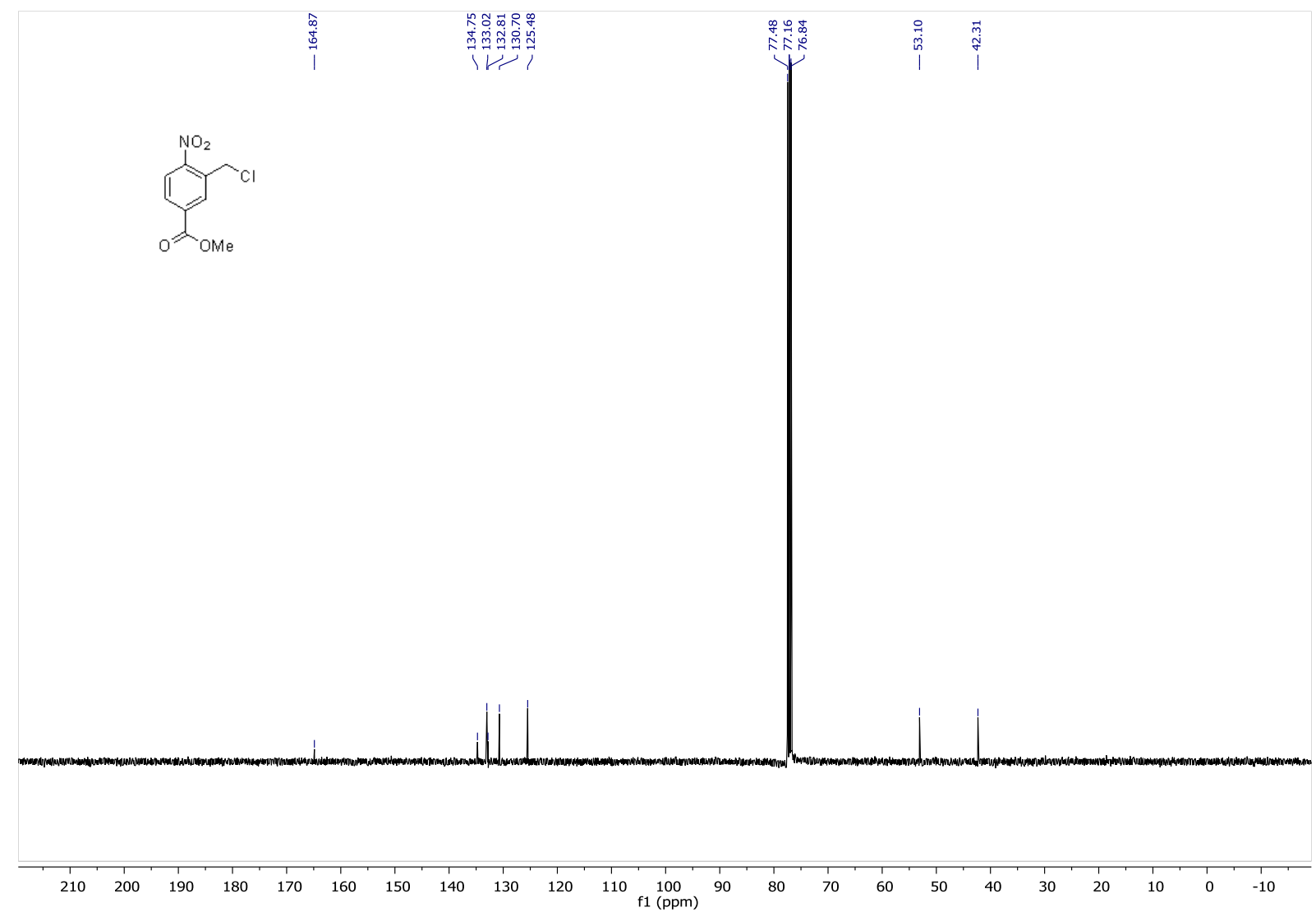

2r, ${ }^{1} \mathrm{H} \mathrm{NMR}\left(\mathrm{CDCl}_{3}, 400 \mathrm{MHz}\right)$

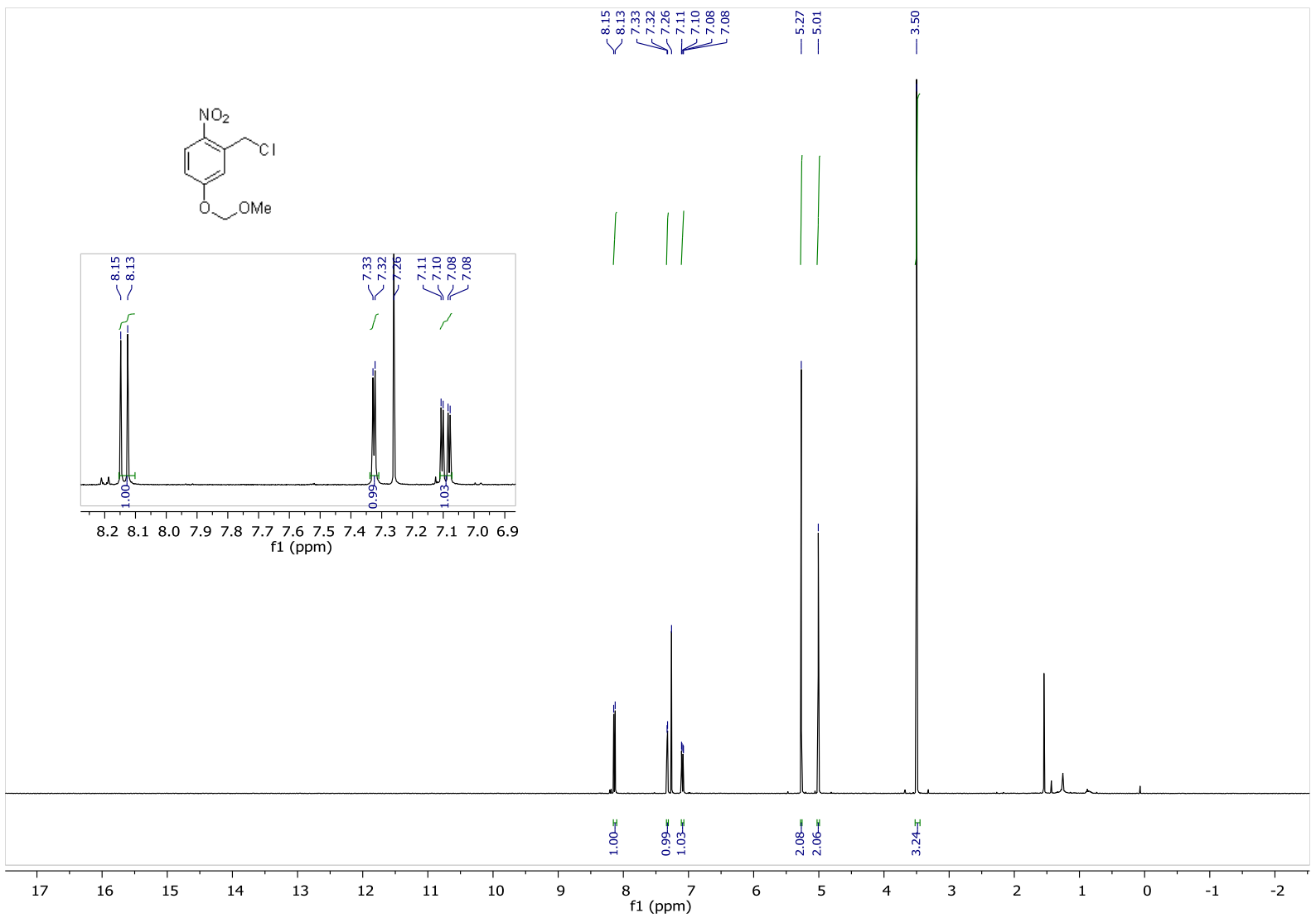

2r, ${ }^{13} \mathrm{C} \mathrm{NMR}\left(\mathrm{CDCl}_{3}, 100 \mathrm{MHz}\right)$ 


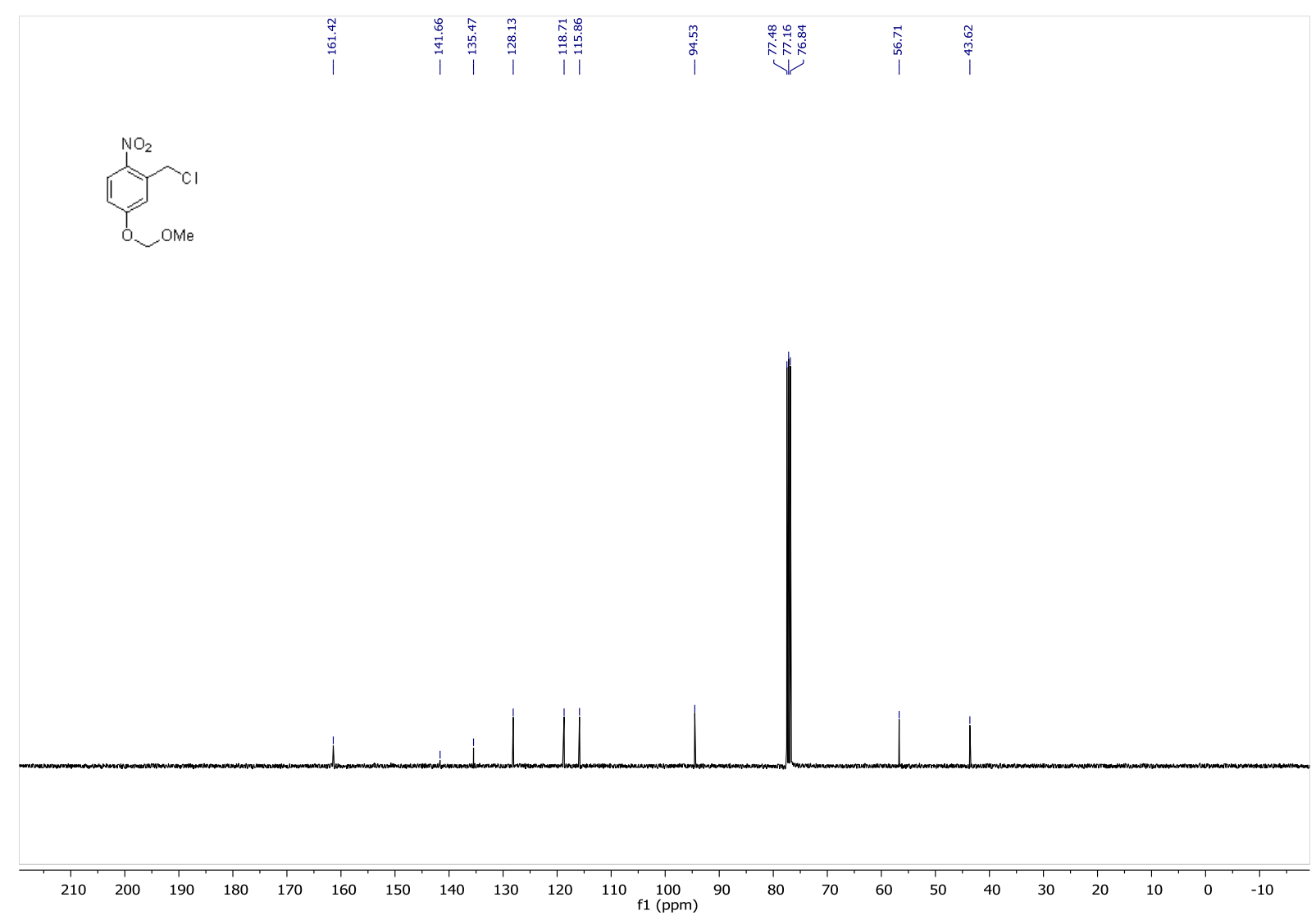

2s, ${ }^{1} \mathrm{H} \mathrm{NMR}\left(\mathrm{CDCl}_{3}, 400 \mathrm{MHz}\right)$

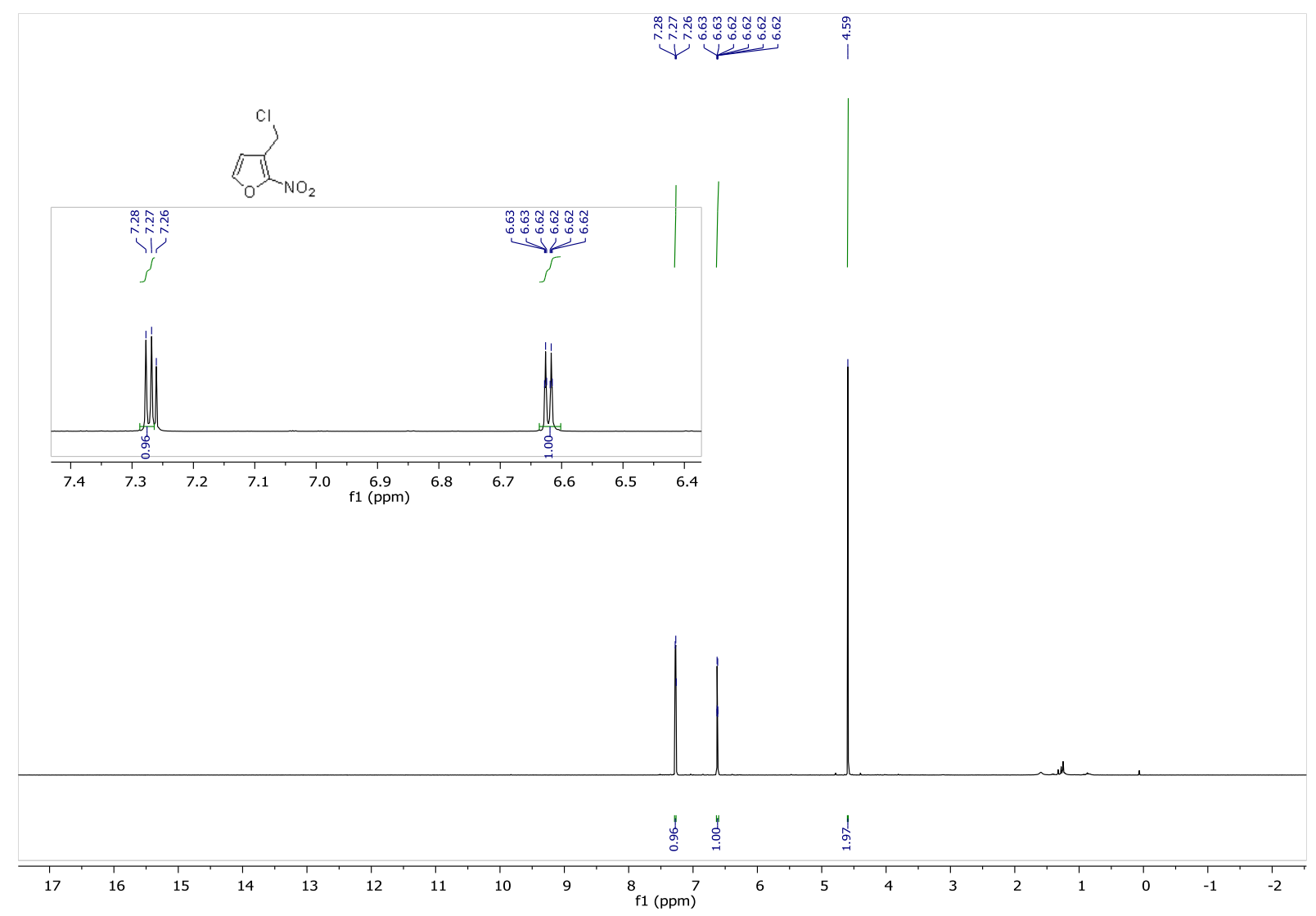

2s, ${ }^{13} \mathrm{C} \mathrm{NMR}\left(\mathrm{CDCl}_{3}, 100 \mathrm{MHz}\right)$ 

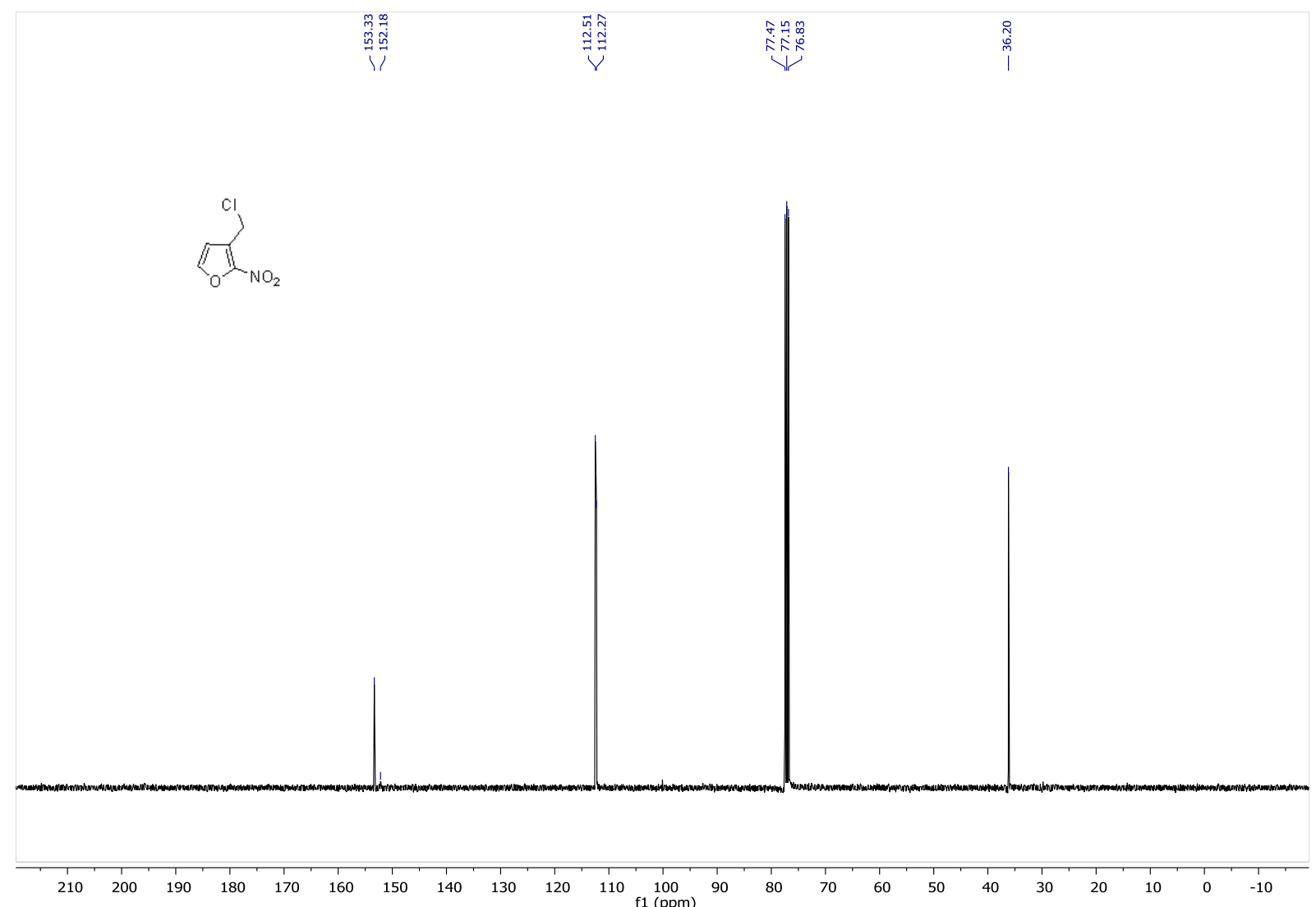

2t, ${ }^{1} \mathrm{H} \mathrm{NMR}\left(\mathrm{CDCl}_{3}, 400 \mathrm{MHz}\right)$

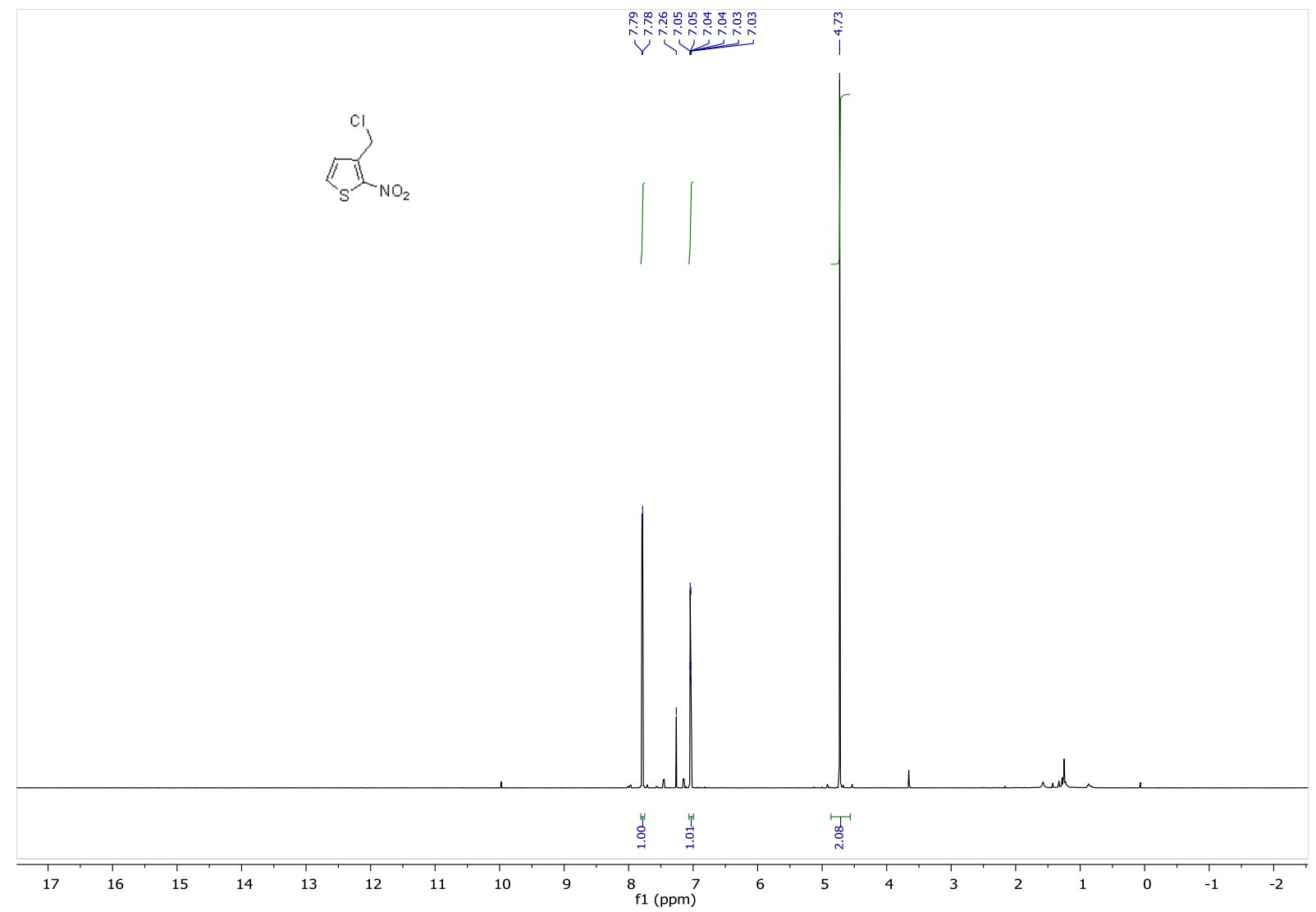

2t, ${ }^{13} \mathrm{C}$ NMR $\left(\mathrm{CDCl}_{3}, 100 \mathrm{MHz}\right)$ 

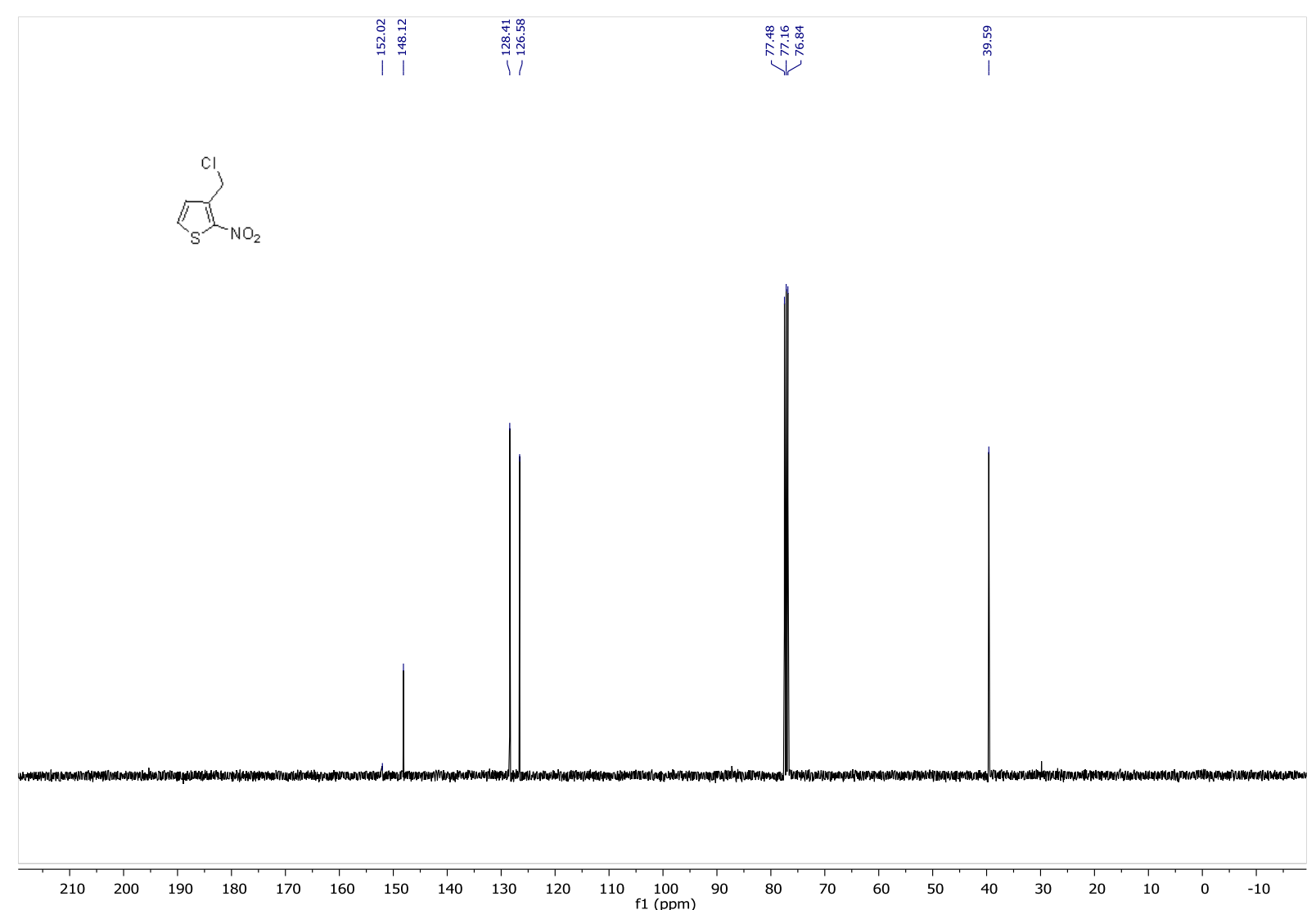

4a, ${ }^{1} \mathrm{H} \mathrm{NMR}\left(\mathrm{CDCl}_{3}, 400 \mathrm{MHz}\right)$

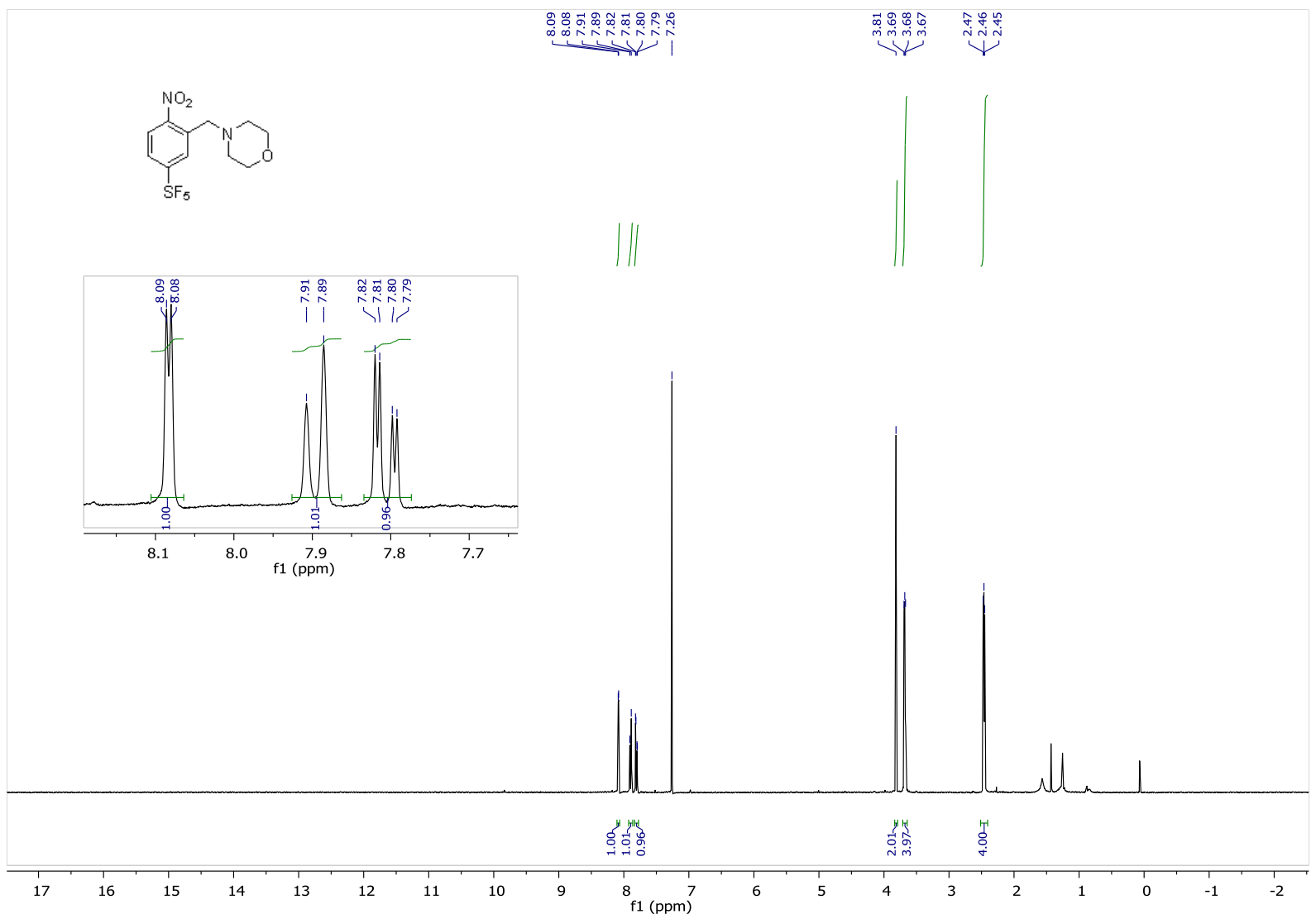

4a, ${ }^{13} \mathrm{C} \mathrm{NMR}\left(\mathrm{CDCl}_{3}, 100 \mathrm{MHz}\right)$ 


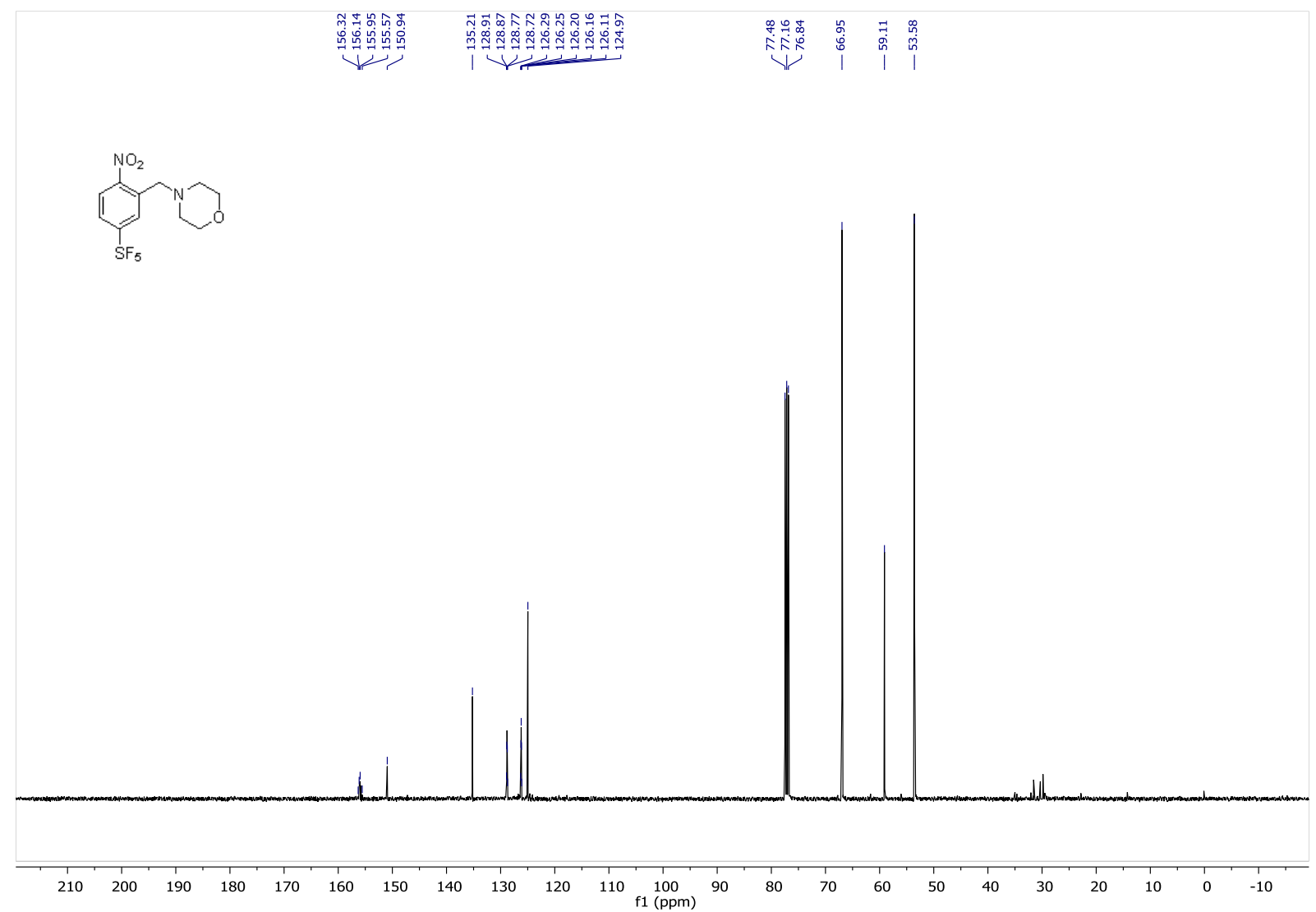

4a, ${ }^{19} \mathrm{~F} \mathrm{NMR}\left(\mathrm{CDCl}_{3}, 376 \mathrm{MHz}\right)$

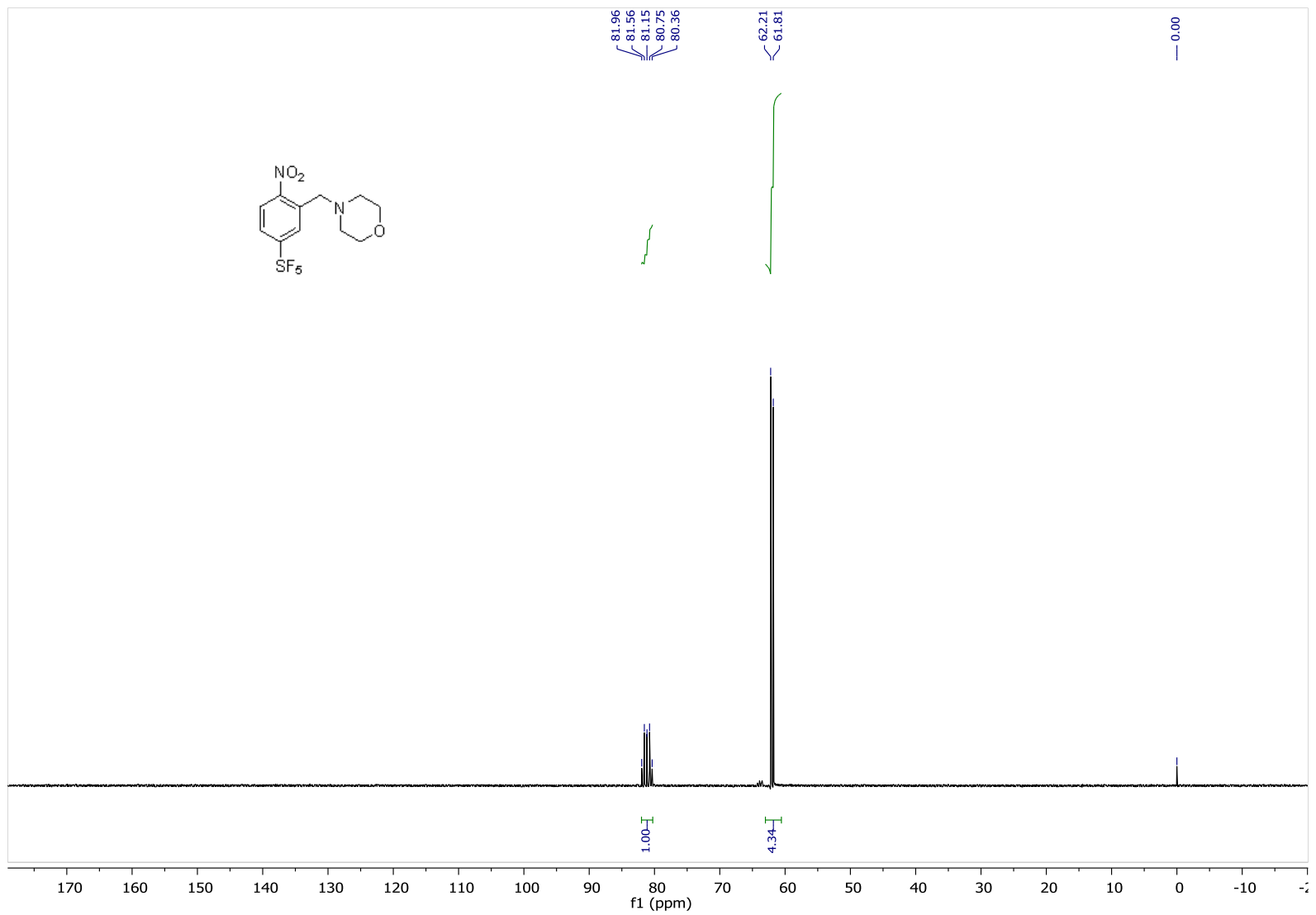

4b, ${ }^{1} \mathrm{H} \mathrm{NMR}\left(\mathrm{CDCl}_{3}, 400 \mathrm{MHz}\right)$ 


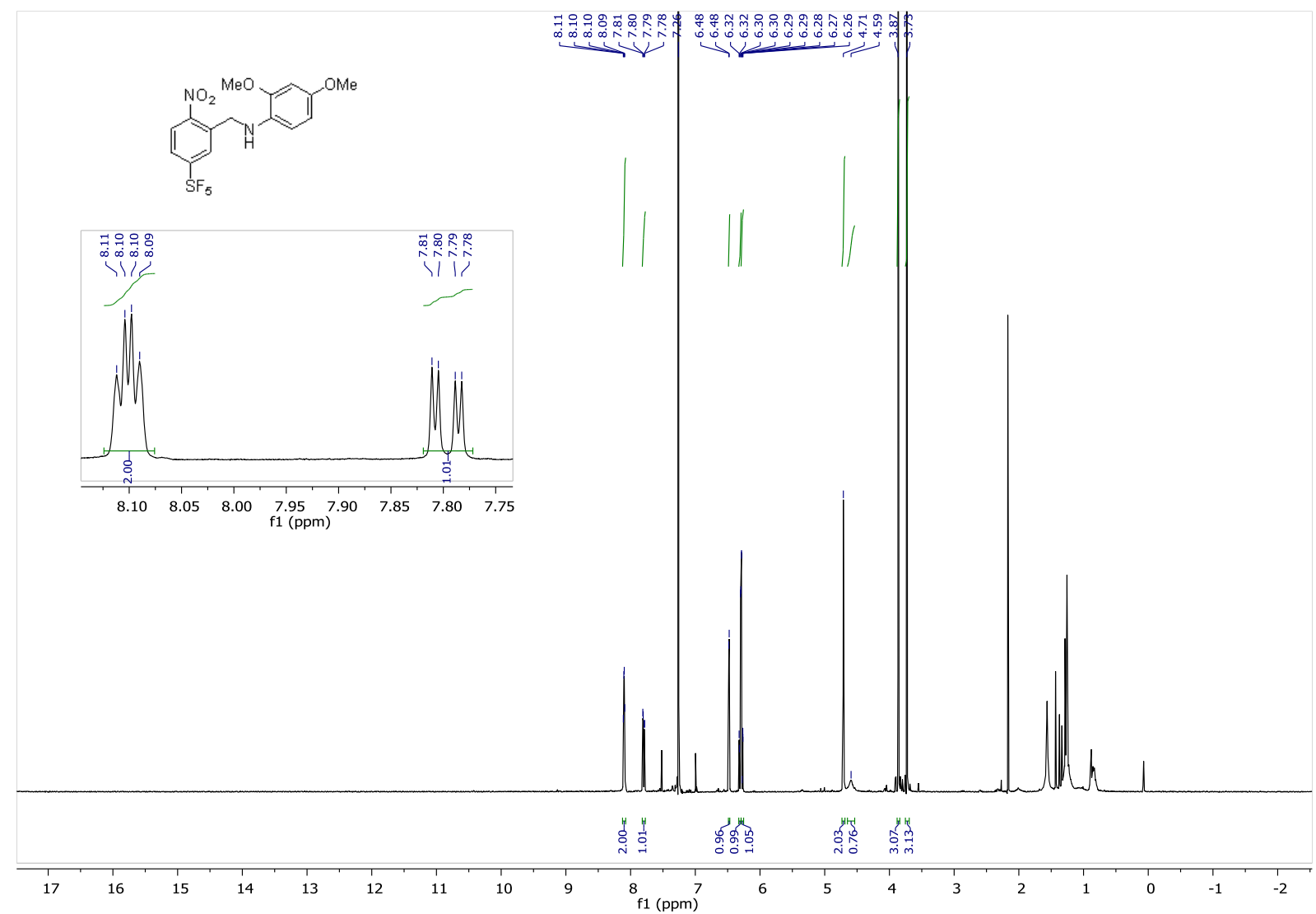

4b, ${ }^{13} \mathrm{C} \mathrm{NMR}\left(\mathrm{CDCl}_{3}, 100 \mathrm{MHz}\right)$

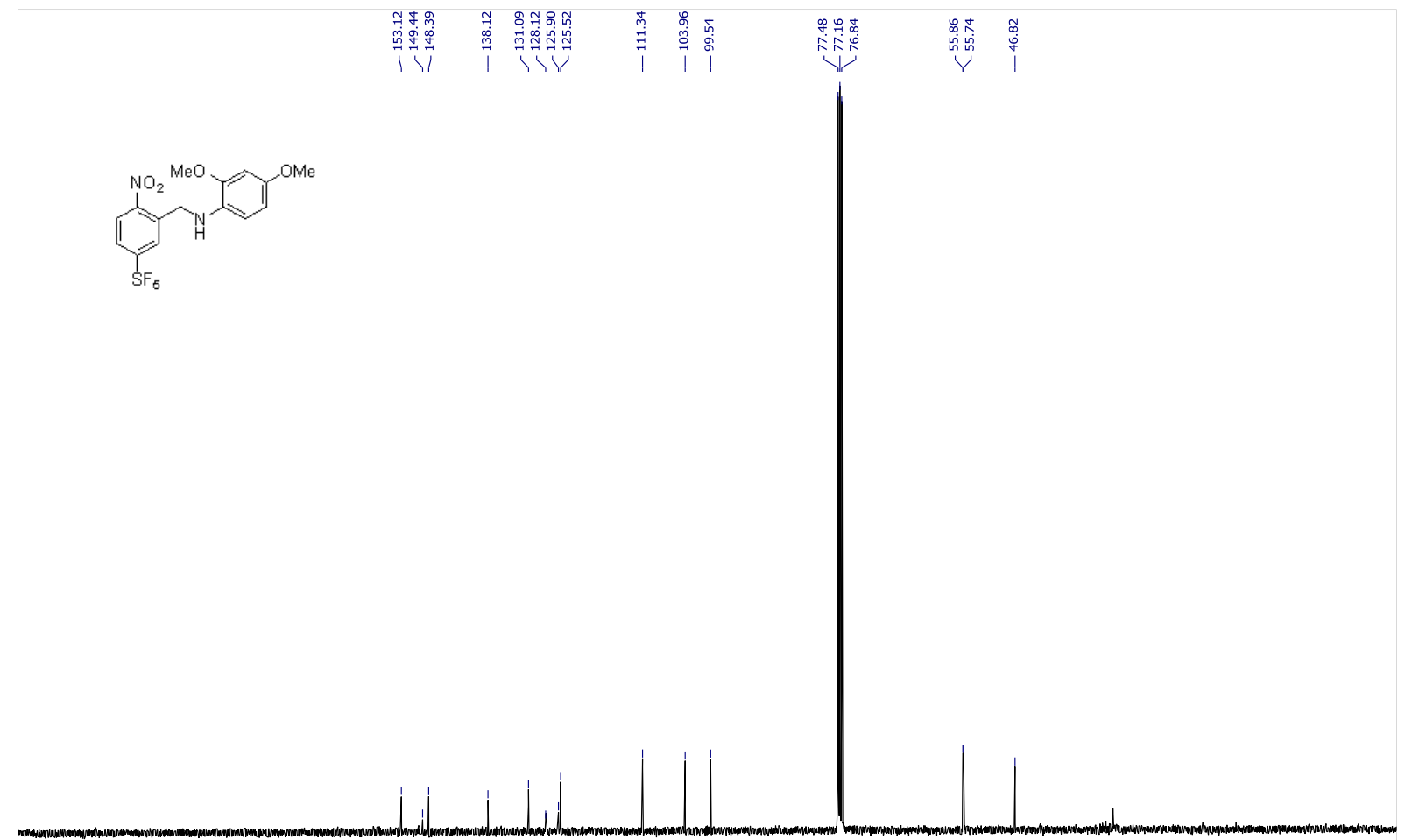

$\begin{array}{llllllllllllllllllllllllllll}210 & 200 & 190 & 180 & 170 & 160 & 150 & 140 & 130 & 120 & 110 & 100 & 90 & 80 & 70 & 60 & 50 & 40 & 30 & 20 & 10 & 0 & -10\end{array}$

4b, ${ }^{19} \mathrm{~F} \mathrm{NMR}\left(\mathrm{CDCl}_{3}, 376 \mathrm{MHz}\right)$ 


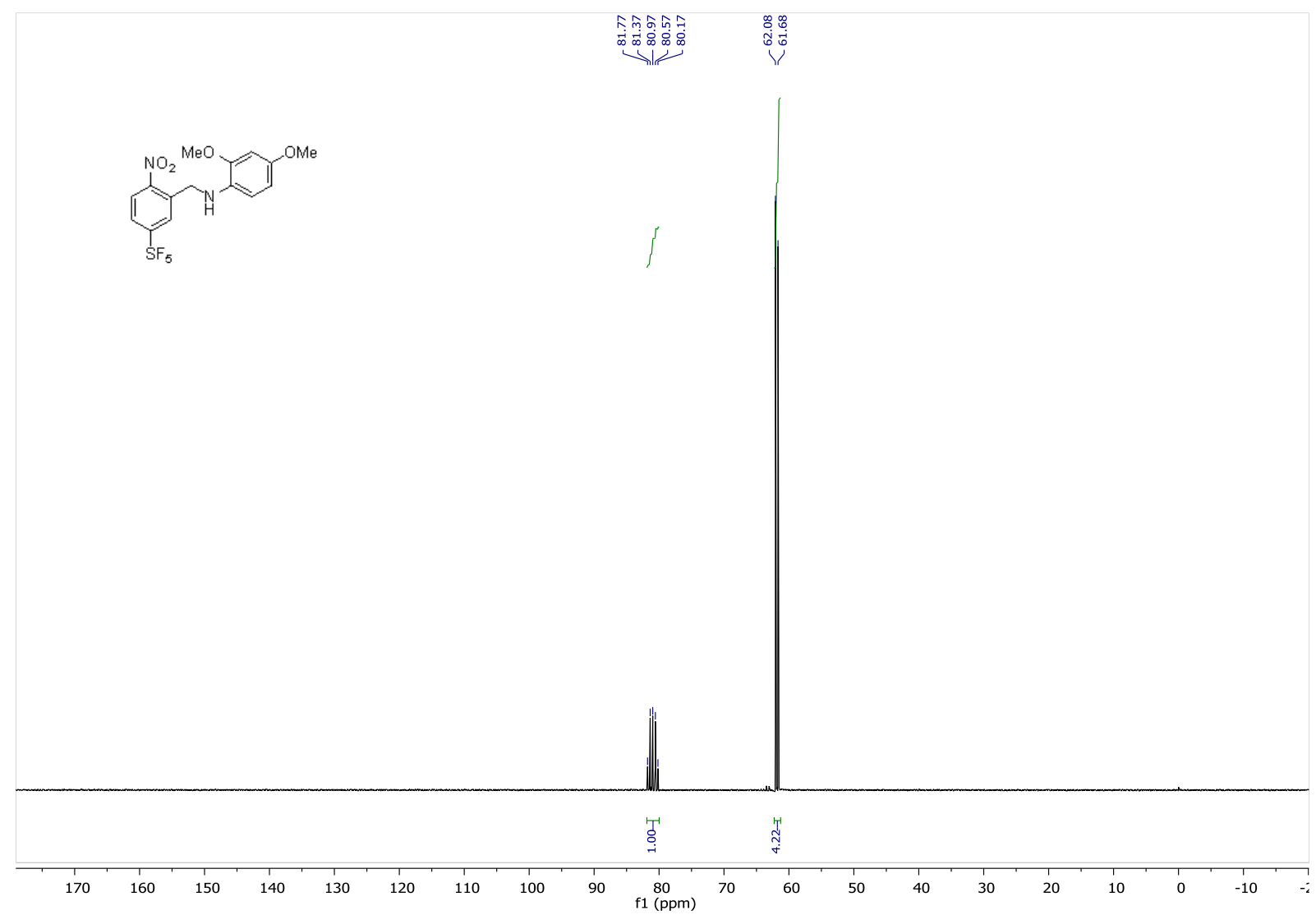

4c, ${ }^{1} \mathrm{H}$ NMR (acetone- $d_{6}, 400 \mathrm{MHz}$ )

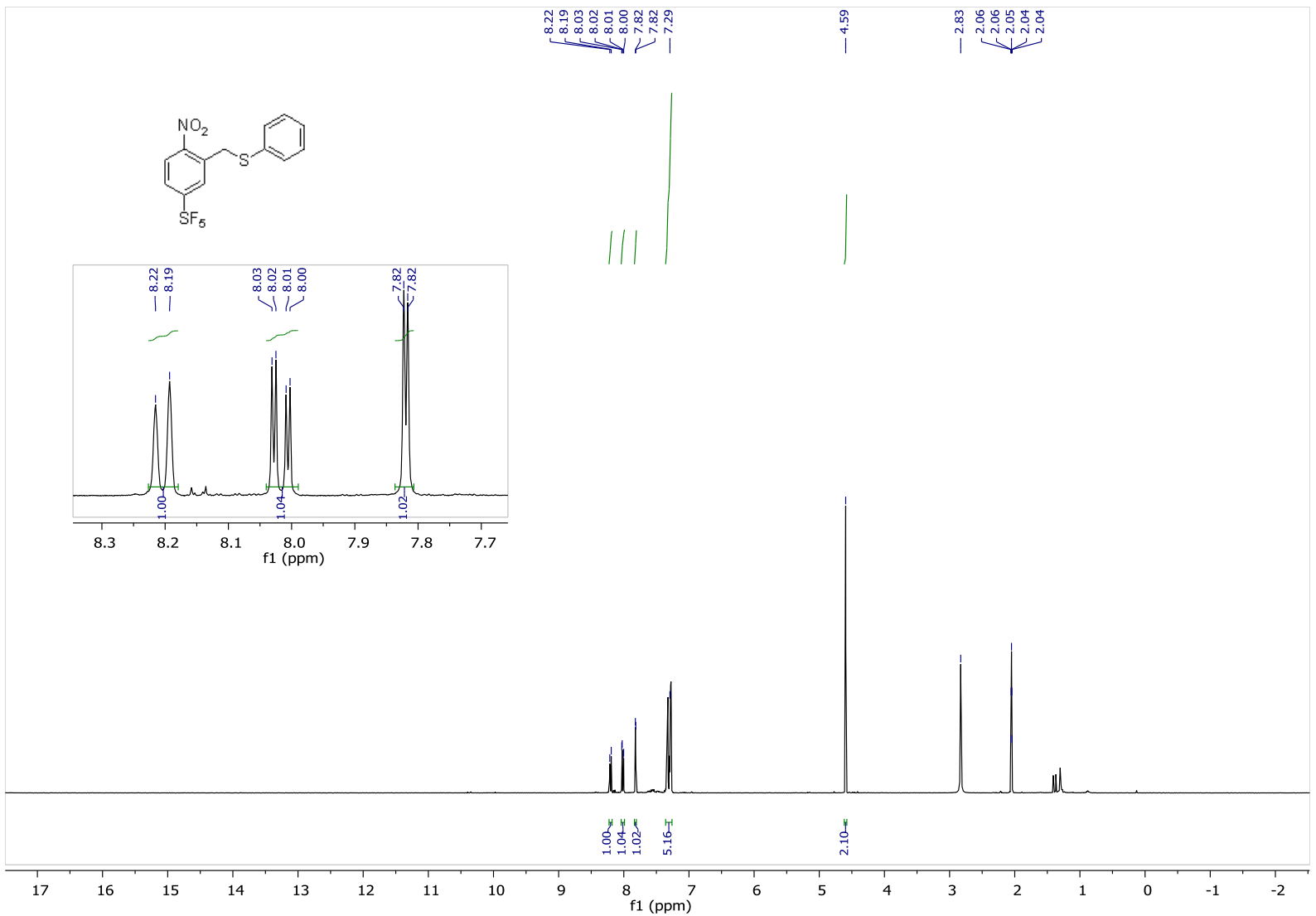

4c, ${ }^{13} \mathrm{C}$ NMR (acetone- $d_{6}, 100 \mathrm{MHz}$ ) 

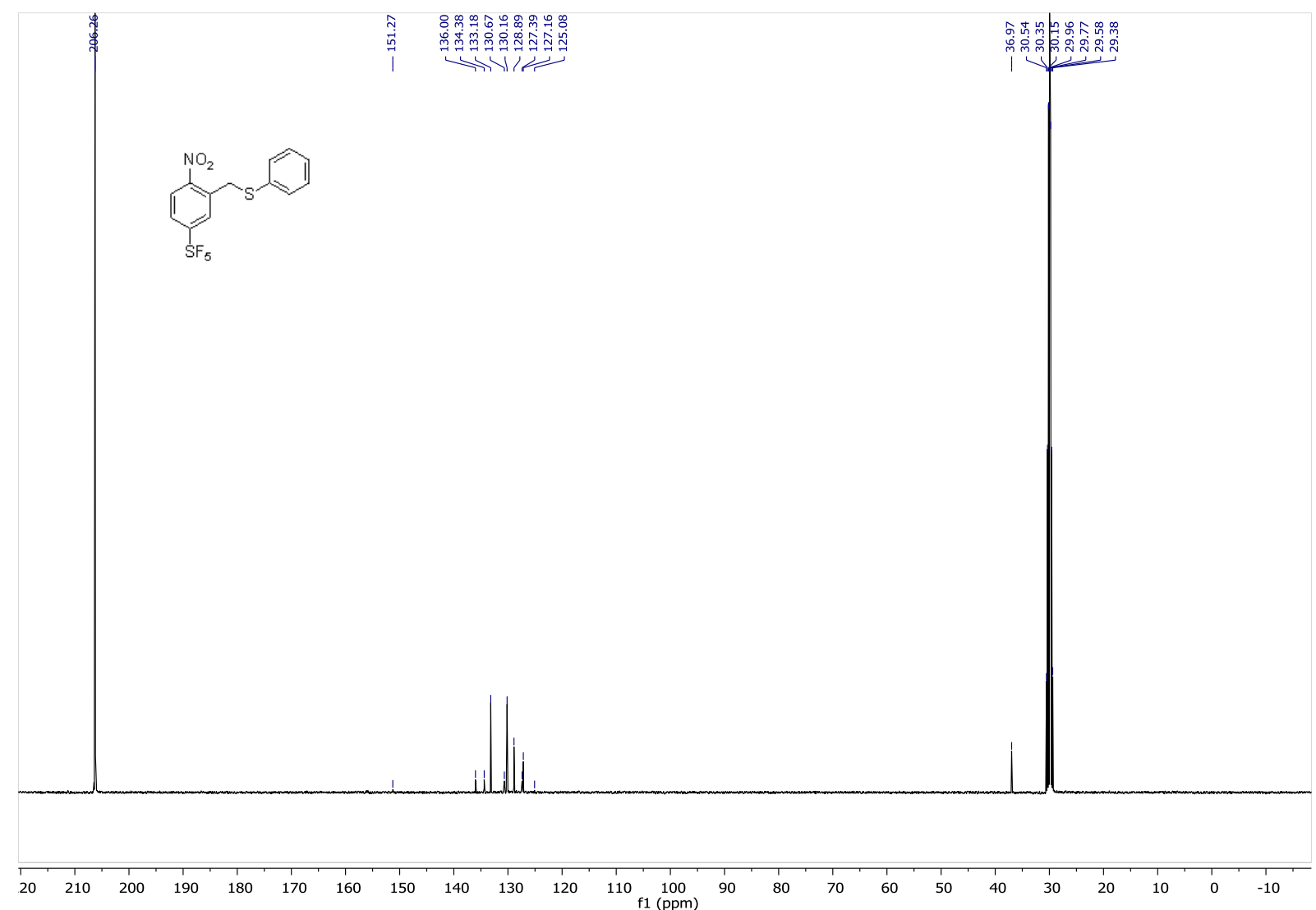

$4 \mathbf{c}^{19} \mathrm{~F}$ NMR (acetone- $d_{6}, 376 \mathrm{MHz}$ )

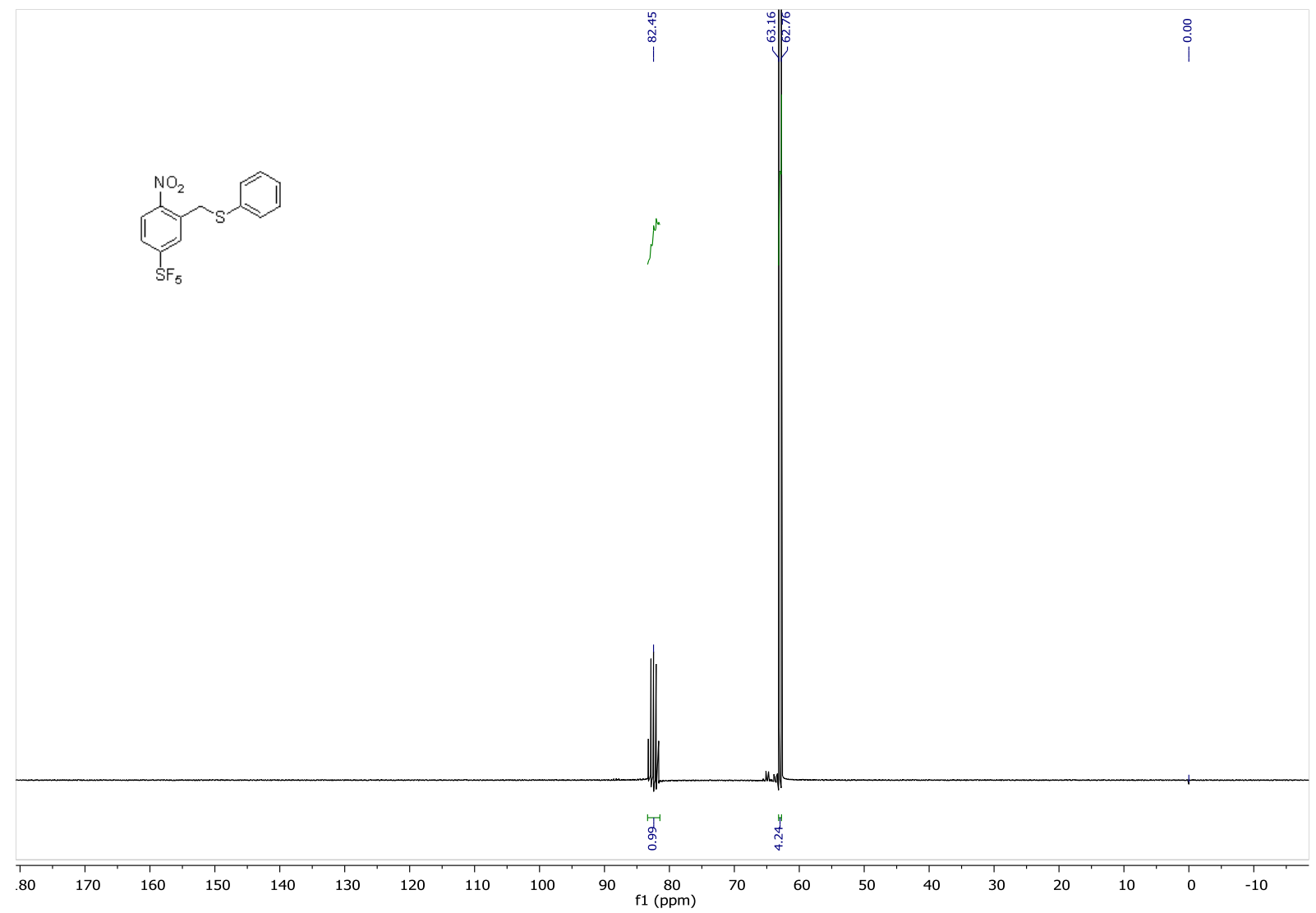

5b, ${ }^{1} \mathrm{H} \mathrm{NMR}\left(\mathrm{CDCl}_{3}, 400 \mathrm{MHz}\right)$ 


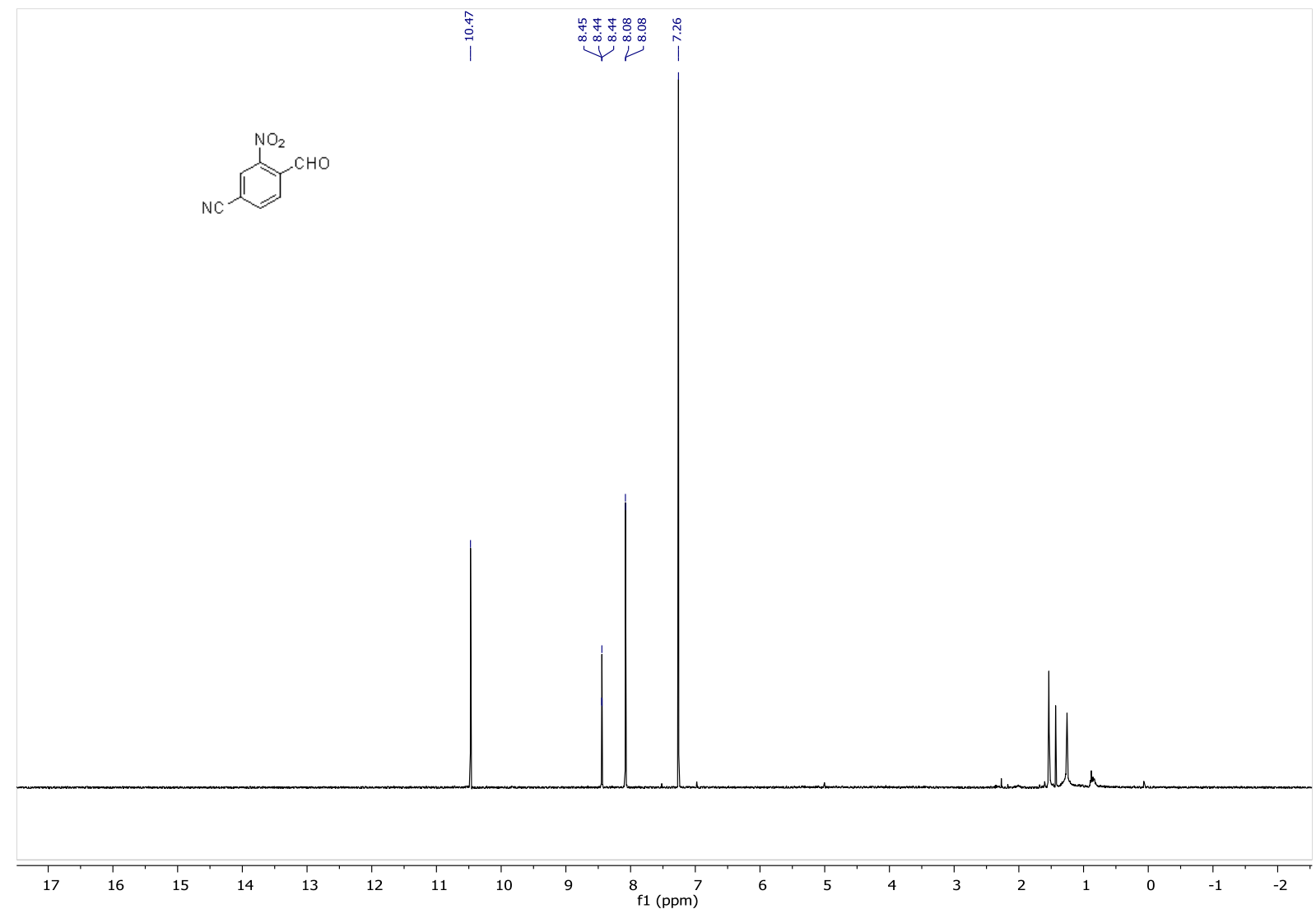

$\mathbf{5 b},{ }^{13} \mathrm{C} \mathrm{NMR}\left(\mathrm{CDCl}_{3}, 100 \mathrm{MHz}\right)$

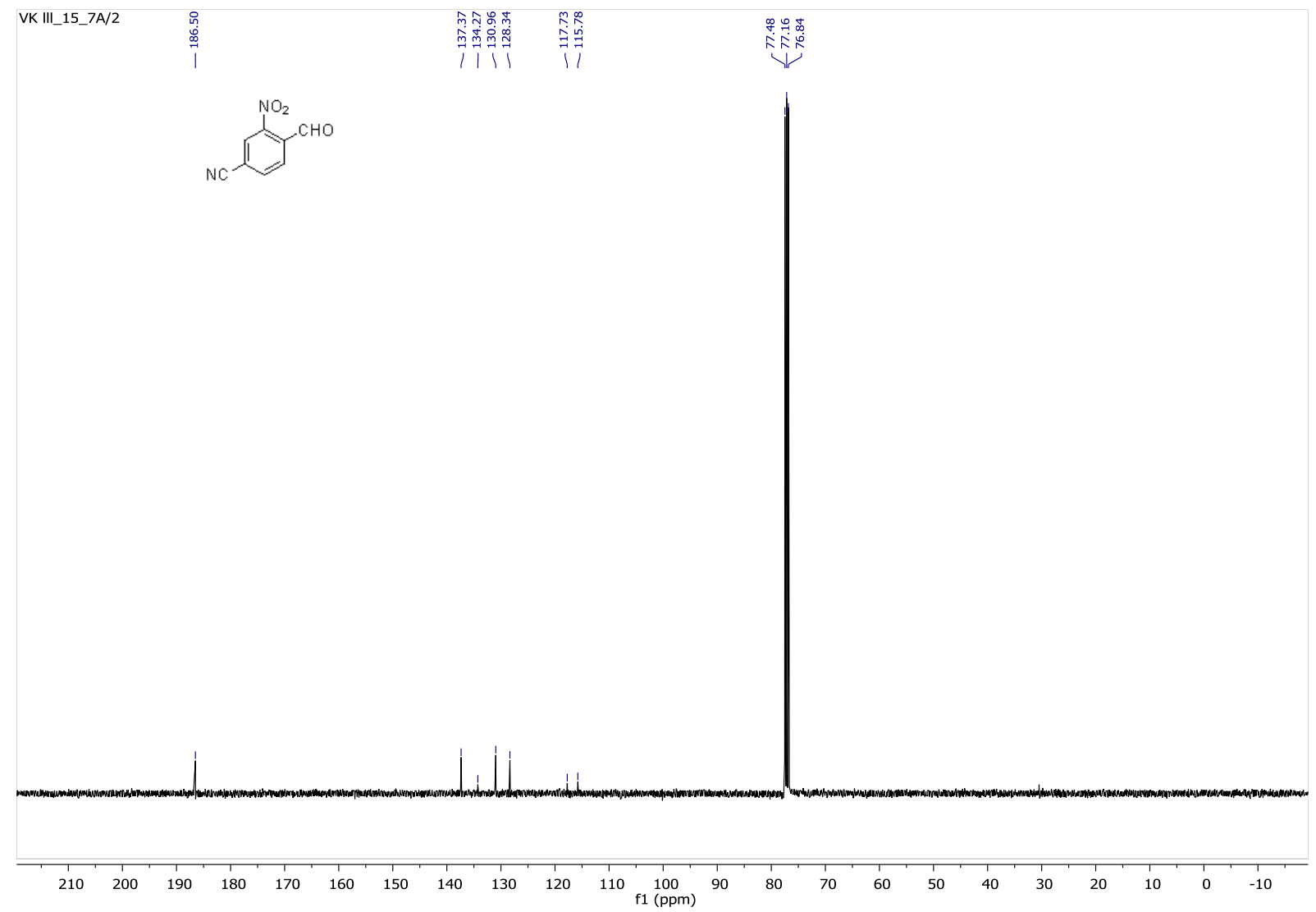

5c, ${ }^{1} \mathrm{H} \mathrm{NMR}\left(\mathrm{CDCl}_{3}, 400 \mathrm{MHz}\right)$ 


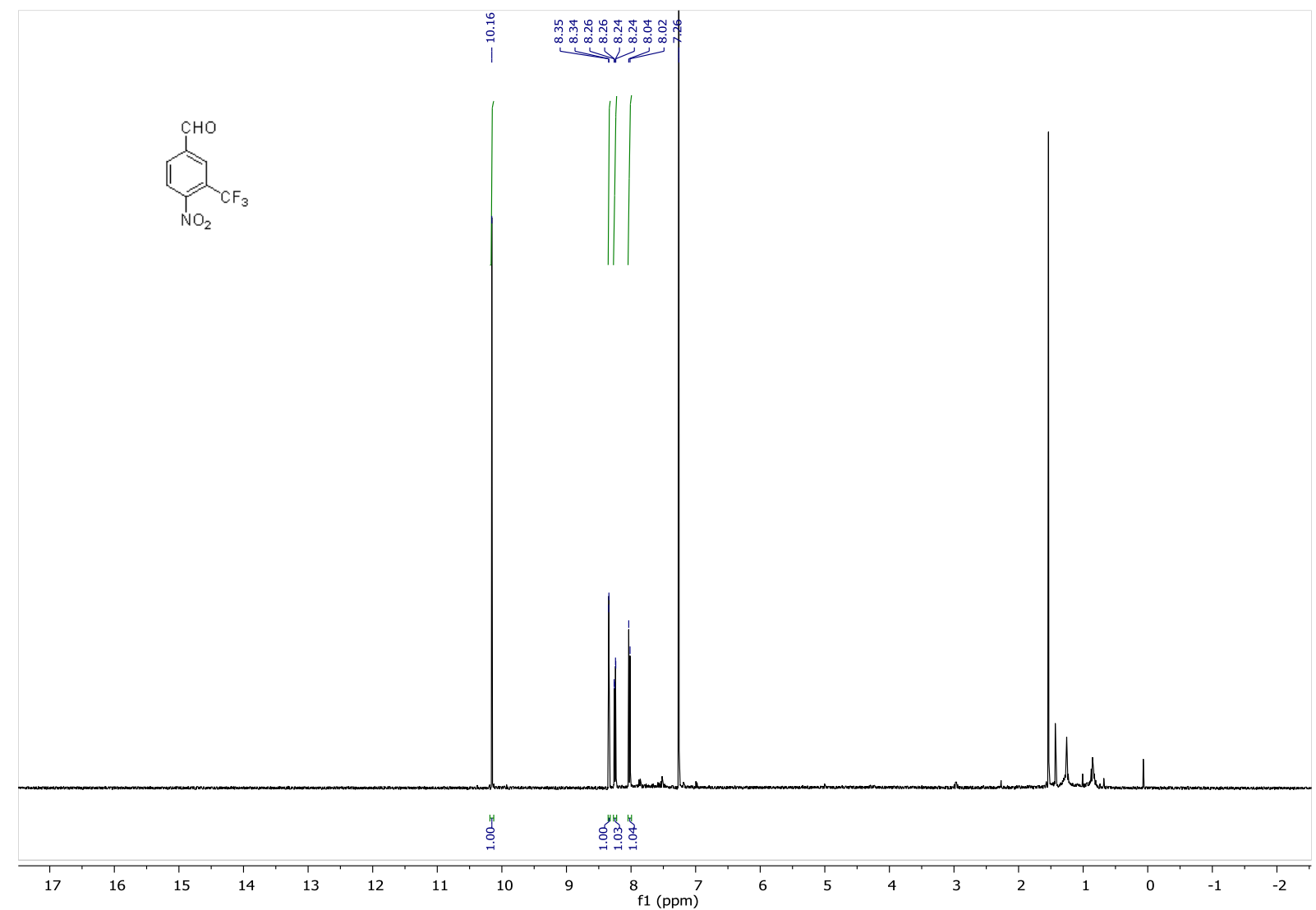

5c, ${ }^{13} \mathrm{C}$ NMR (APT, $\left.\mathrm{CDCl}_{3}, 100 \mathrm{MHz}\right)$

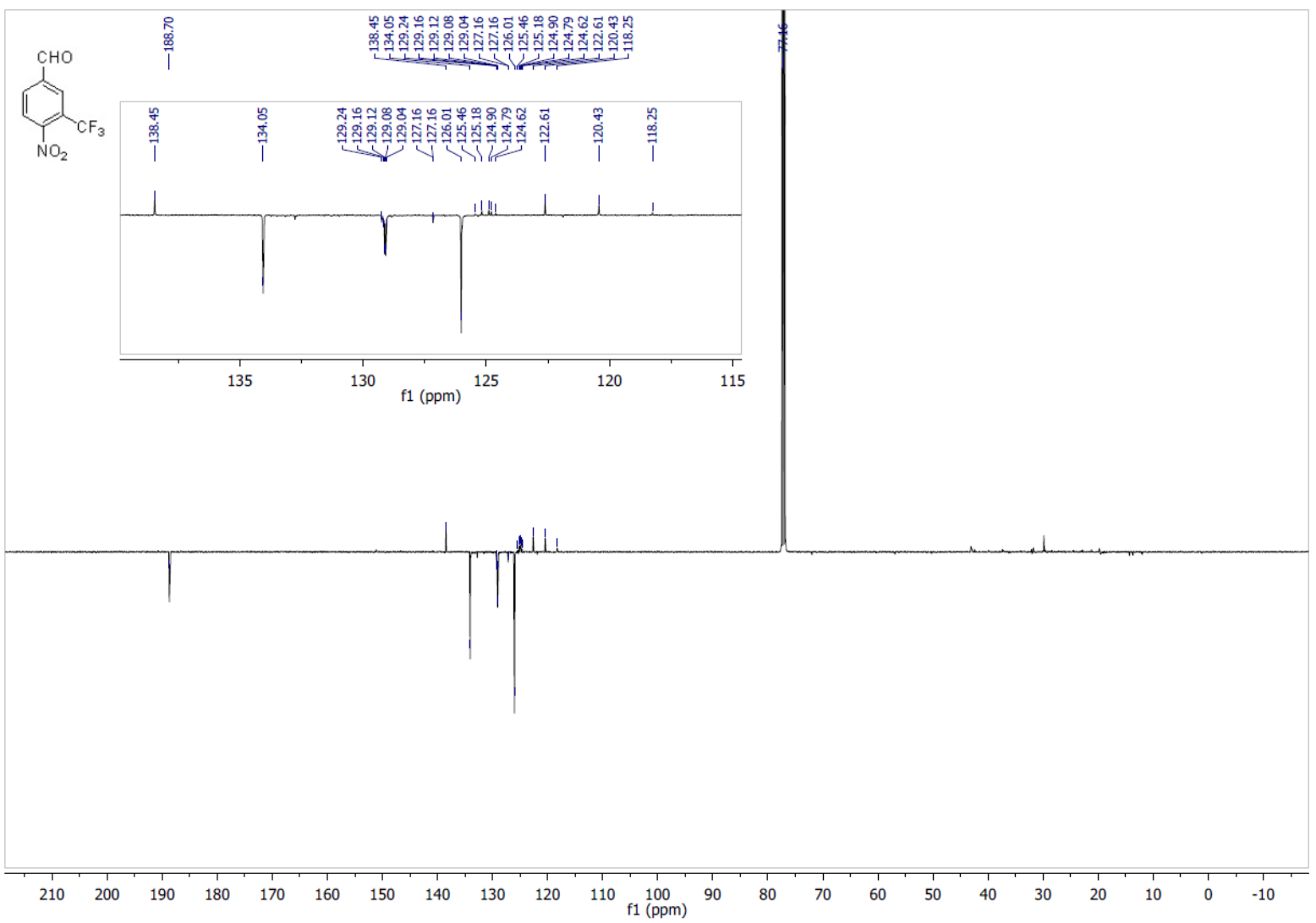

5c, ${ }^{19} \mathrm{~F} \mathrm{NMR}\left(\mathrm{CDCl}_{3}, 376 \mathrm{MHz}\right)$ 


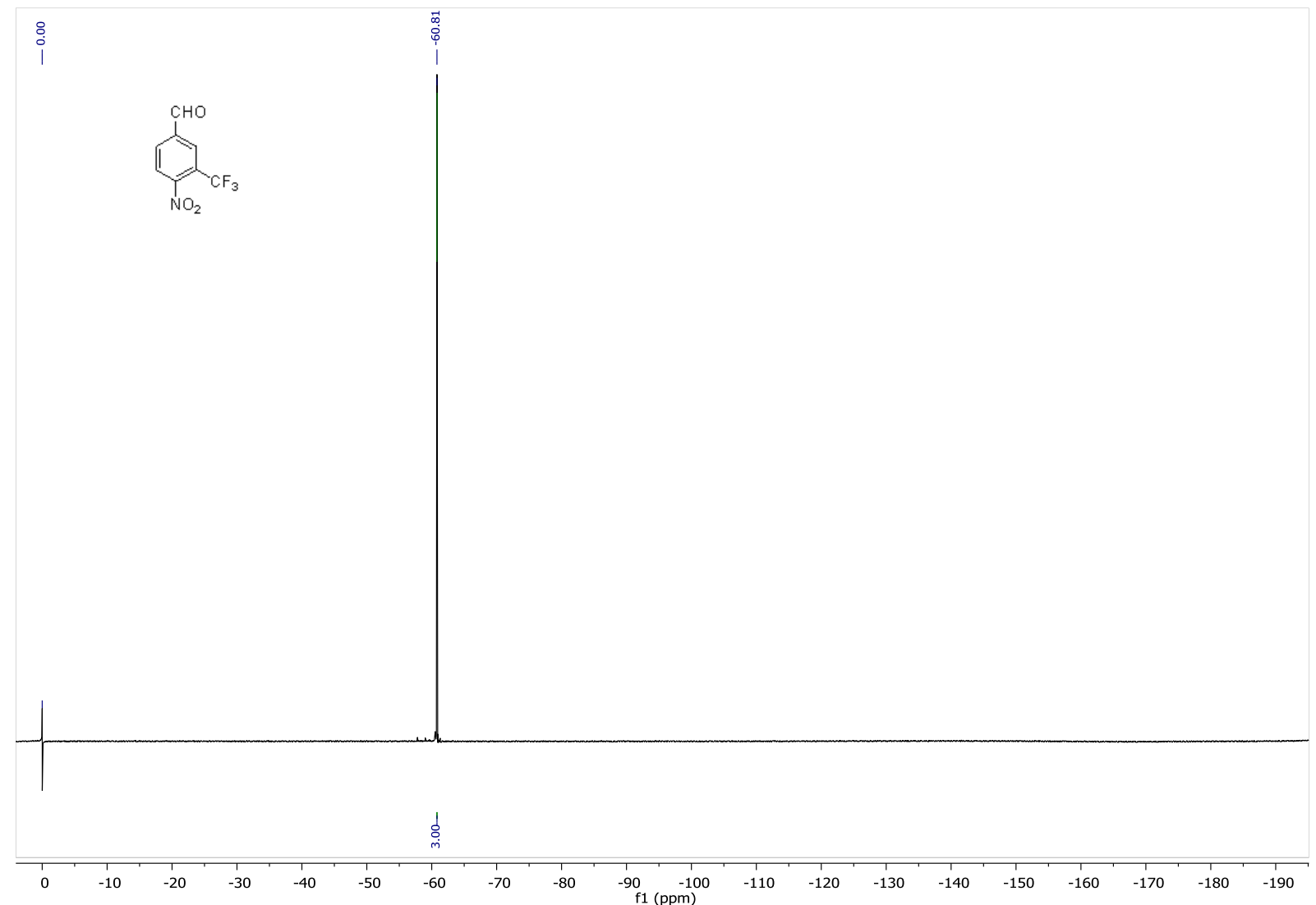

